\title{
Variedades centrais, desingularização e existência de superfícies Canard
}

Gerard John Alva Morales

DISSERTAÇÃO APRESENTADA

$\mathrm{AO}$

INSTITUTO DE MATEMÁTICA E ESTATÍSTICA

DA

UNIVERSIDADE DE SÃO PAULO

PARA

OBTENÇÃO DO TÍTULO DE MESTRE

EM

CIÊNCIAS

Área de Concentração: Matemática

Orientador: Prof.Dr. Daniel Cantergiani Panazzolo

São Paulo/SP-Dezembro-2005 


\title{
Variedades centrais, desingularização e existência de superfícies Canard
}

\author{
Este exemplar corresponde à redação final \\ da dissertação devidamente corrigida e defendida \\ por Gerard John Alva Morales e aprovada \\ pela comissão julgadora. \\ São Paulo/SP 9-Dezembro-2005
}

Banca Examinadora:

Prof.Dr. Daniel Cantergiani Panazzolo(Orientador) -IME-USP

Prof.Dr. Jorge Manuel Sotomayor Tello -IME-USP

Prof.Dr. Paulo Ricardo da Silva -IBILCE-UNESP 
Dedico este trabalho a meus pais Marco e Georgina e a minha irmã Miriam 


\section{Agradecimento}

Agradeço:

À Deus, pela oportunidade que me deu de viver conforme a sua bondade.

Ao meu orientador, Prof.Dr. Daniel Cantergiani Panazzolo, pela oportunidade que me deu de conhecer a Matemática sutil e elegante que ele estuda e também pela confiança que depositou no meu esforço e pelas valiosas sugestões aportadas.

Aos professores do Instituto de Matemática e Estatística(IME), em particular aos professores, Dr. Jorge Manuel Sotomayor Tello, pelos conhecimentos e sugestões aportadas nesta área de pesquisa, Dr. Luiz Augusto Fernandes de Oliveira, por ter me encaminhado no inicio do mestrado e participado na minha formação matemática.

Aos professores:

Elói Medina Galego, José Antonio Verderesi, Luiz Augusto Fernandes de Oliveira, Antonio Carlos Asperti, Odilon Otávio Luciano, Antonio Carlos Brolezzi, Henrique Salvador S. Panzarelli, Martha Salermo, Zara Issa Abud; por terem permitido participar das Monitorias semestrais do IME, Estágios em matemática e pelo apoio moral, o qual foi relevante na realização do Mestrado.

A minha família, pelo apoio moral, confiança e a força incondicional que me proporcionaram durante o mestrado.

$\mathrm{E}$ a todos os colegas e amigos, pela amizade. 


\section{Resumo}

Neste trabalho usaremos a teoria das Perturbações Singulares e das Variedades Centrais para estudar o fenômeno Canard numa família de campos de vetores no plano da forma

$$
X_{\varepsilon, \alpha}(x, y)=\varepsilon \frac{\partial}{\partial x}+y F(x, y, \varepsilon, \alpha) \frac{\partial}{\partial y}, \quad \alpha \in \mathbb{R}^{n}, \varepsilon \in \mathbb{R}_{+}
$$

onde $F$ é uma função analítica tal que $F(x, y, 0,0)=B x^{k}+O\left(x^{k+1}, y\right)$, para alguma constante $B \neq 0$. Este fenômeno consiste na existência de famílias de soluções regulares da equação diferencial, para valores do parâmetro $\varepsilon>0$, que permanecem próximas de um conjunto normalmente hiperbólico repulsor quando tomamos $\varepsilon$ tendendo a zero. Para valores $k>0$, a singularidade na origem é usualmente chamada de turning point. 


\section{Abstract}

In this work we use the theory of Singular Perturbations and Center Manifolds to study the Canard phenomena in a family of planar vector fields of the form

$$
X_{\varepsilon, \alpha}(x, y)=\varepsilon \frac{\partial}{\partial x}+y F(x, y, \varepsilon, \alpha) \frac{\partial}{\partial y}, \quad \alpha \in \mathbb{R}^{n}, \varepsilon \in \mathbb{R}_{+}
$$

where $F$ is an analytic function such that $F(x, y, 0,0)=B x^{k}+O\left(x^{k+1}, y\right)$, for some constant $B \neq 0$. This phenomena consists in the existence of families of regular solutions of the differential equation for values of the parameter $\varepsilon>0$, which remain near of a normally hyperbolic repelling set when we take $\varepsilon$ tending to zero. For $k>0$, the singularity at the origin is usually called a turning point. 


\section{Conteúdo}

1 Introdução $\quad 9$

2 Conceitos Preliminares $\quad 15$

2.1 Funções Especiais . . . . . . . . . . . . . . . . . . . . . 15

2.1.1 Aplicação Blowing-up . . . . . . . . . . . 15

2.1.2 Extensões Blowing-up $C^{k} \ldots \ldots \ldots \ldots$

2.1 .3 Funções $\infty$-Flat. . . . . . . . . . . . . . . 20

2.1.4 Blow-down $\infty$-Flat . . . . . . . . . . . . . 21

2.2 Campos Singularmente Perturbados . . . . . . . . . . 24

2.2.1 Classificação das singularidades para $X_{\varepsilon, a} \ldots \ldots 25$

2.3 Hipóteses Gerais . . . . . . . . . . . . . . . . . 30

2.3.1 Hipóteses para $(X, \Gamma) \ldots \ldots \ldots$

2.3.2 Hipóteses de Transversalidade para $(X, 0) \ldots \ldots$

2.3.3 Blowing-up Principal . . . . . . . . . . . . . . . 31

3 Variedades Centrais sobre $\Gamma \backslash \operatorname{Deg}(X) \quad 33$

3.1 Linearização . . . . . . . . . . . . . . . . . . . . . . . 33

3.2 Variedades Centrais Formais . . . . . . . . . . . . . 36

3.3 Variedade Central Dinâmica . . . . . . . . . . . . . . . 40

4 Geometria no espaço fase de $\bar{X} \quad 45$

4.1 Folheação regular sobre a carta $K_{\bar{\varepsilon}} \ldots \ldots \ldots \ldots \ldots$

4.2 Estudo nas cartas $K_{ \pm \bar{x}_{\varepsilon}} \ldots \ldots \ldots \ldots \ldots$

4.3 Relação entre as cartas $K_{\bar{\varepsilon}}, K_{ \pm \bar{x}_{\varepsilon}} \quad \ldots \ldots \ldots \ldots . \ldots \ldots$

5 Dinâmica Assintótica sobre a carta $K_{\bar{\varepsilon}} \quad 57$

$5.1 \quad \mathrm{O}$ caso $R_{A, 0}(0) \ldots \ldots \ldots \ldots \ldots \ldots \ldots \ldots \ldots$

5.1 .1 Caso $\mathcal{B}_{0}>0 \ldots \ldots \ldots \ldots \ldots$

5.1 .2 Caso $\mathcal{B}_{0}<0 \ldots \ldots \ldots \ldots \ldots \ldots \ldots$

$5.2 \quad \mathrm{O}$ caso $R_{A, B}(0) \ldots \ldots \ldots \ldots \ldots \ldots \ldots$ 
5.2 .1 Caso $\mathcal{B}_{0}>0 \ldots \ldots \ldots \ldots \ldots \ldots \ldots$

5.2 .2 Caso $\mathcal{B}_{0}<0 \ldots \ldots \ldots \ldots \ldots 6$

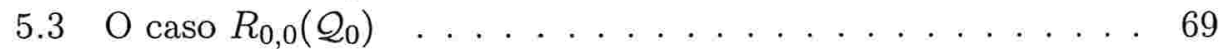

5.3 .1 Caso $\mathrm{p}+1$ par . . . . . . . . . . . . . . . 69

5.3 .2 Caso p+1 ímpar . . . . . . . . . . . . . 72

5.4 Caso $R_{A, B}\left(\mathcal{Q}_{0}\right) \ldots \ldots \ldots \ldots \ldots \ldots \ldots$

6 Dinâmica sobre as cartas $K_{ \pm \bar{x}_{\varepsilon}} \quad 81$

6.1 Região atratora . . . . . . . . . . . . . . . . . . 84

6.2 Extensão Dinâmica das variedades Centrais . . . . . . . . . 89

6.3 Estimativas para $\mathcal{O}\left(W_{-}, W_{+}\right) \ldots \ldots \ldots \ldots$

$\begin{array}{lll}7 & \text { Superfícies Canard } & \mathbf{9 7}\end{array}$

7.1 Blowing-up e Blowing-down de $\mathcal{O}\left(W_{-}, W_{+}\right) \ldots \ldots \ldots 9$

7.2 Existência de Superfícies Canard . . . . . . . . . . . 102

$7.2 .1\left(\mathcal{O}_{\widetilde{\Gamma}}, w\right)$ nos casos $(s, s),(u, u) \ldots \ldots \ldots 102$

$7.2 .2\left(\mathcal{O}_{\widetilde{\Gamma}}, w\right)$ sobre o caso $(u, s) \ldots \ldots \ldots \ldots$

$7.2 .3\left(\mathcal{O}_{\widetilde{\Gamma}}, w\right)$ sobre o caso $(s, u) \ldots \ldots \ldots \ldots$

7.3 Conclusões . . . . . . . . . . . . . . . . . . . . 115

7.4 Cálculo Assintótico . . . . . . . . . . . . . . . 117

7.4.1 Escala de Comparação . . . . . . . . . . . . . . 117

7.4.2 Convergência de Integrais Impróprias relativas a $\mathcal{E}$. . 119

7.5 Equações Diferenciais . . . . . . . . . . . . . . 125 


\section{Capítulo 1}

\section{Introdução}

Nosso principal interesse é o estudo do fenômeno Canard, que pode ser observado nos sistemas de equações diferenciais com perturbações singulares.

A abordagem geométrica que utilizaremos neste trabalho baseia-se no artigo [DP]; que nos permitirá introduzir algumas ferramentas da Teoria Geométrica de Perturbações Singulares; referimos também à literatura [NF], [FDR1,2] e [T].

Nos problemas de Relaxação Oscilação, aparecem tipicamente classes especiais de ciclos limites, cujo comportamento qualitativo recai no estudo de problemas de perturbação singular. Vejamos um exemplo:

Tomemos a famosa equação de Balthasar Van der Pol.

$$
\varepsilon \ddot{x}+\left(x^{2}+x\right) \dot{x}+x-a=0, \quad(\varepsilon, a) \in \mathbb{R}^{+} \times \mathbb{R}, \quad x \in \mathbb{R} .
$$

Esta equação, através de mudanças de coordenadas adequadas e uma reparametrização do tempo, pode ser olhada no plano $(x, y)$ como um campo de vetores

$$
X_{\varepsilon, a}=\left\{\begin{aligned}
\dot{x} & =(y-F(x)) \\
\dot{y} & =\varepsilon(a-x)
\end{aligned}\right.
$$

onde $F(x)=\int_{0}^{x}\left(\xi+\xi^{2}\right) d \xi=x^{2} / 2+x^{3} / 3 ;$ e $X_{0, a}$ é o campo horizontal

$$
X_{0, a}(x, y)=(y-F(x))(\partial / \partial x)
$$

(ver Figura $1.1(\mathrm{a})$ ). A curva cúbica $L=\{y=F(x)\}$ é dita variedade lenta de $X_{\varepsilon, a}$; neste contexto, este sistema é chamado de slow-fast system.

Para certas curvas no espaço de parâmetros

$$
\varepsilon \mapsto a=\gamma(\varepsilon), \quad a(0)=0
$$


é possível mostrar a existência de uma família de curvas fechadas $\left\{\Gamma_{\varepsilon}^{\gamma}\right\}$, tais que $\Gamma_{\varepsilon}^{\gamma}$ é um ciclo limite de $X_{\varepsilon, \gamma(\varepsilon)}$, para $\varepsilon>0$ e

$$
\lim _{\varepsilon \rightarrow 0} \Gamma_{\varepsilon}^{\gamma}=\Gamma_{0}^{\gamma}
$$

(na métrica de Hausdorff). O conjunto $\Gamma_{0}^{\gamma}$ é um subconjunto fechado invariante por $X_{0, a}$, que contém trechos da curva lenta $L$. Fazendo variar $\gamma(\varepsilon)$, obtemos várias formas para este limite $\Gamma_{0}^{\gamma}$, que receberam o nome de Canards(ver Figura 1.1 (b)).

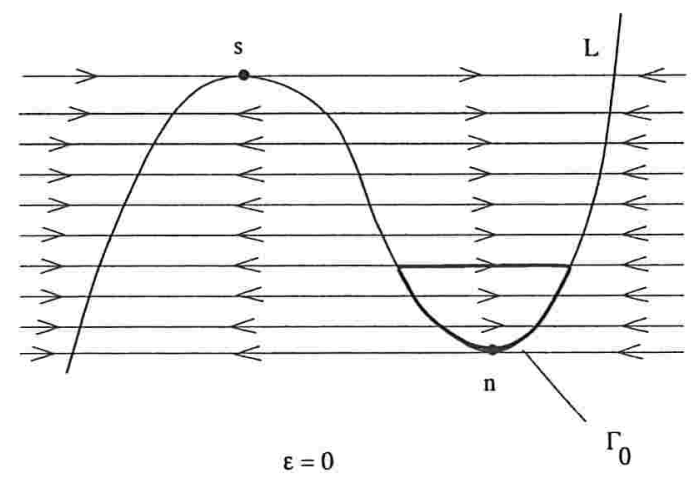

(a)

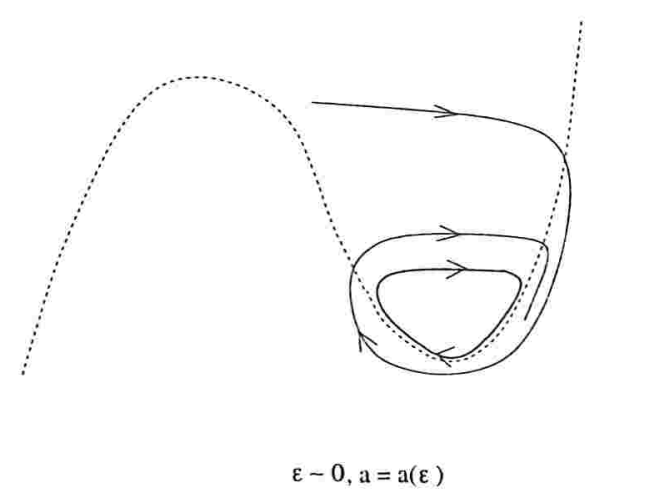

(b)

Figura 1.1: Variedade Lenta da Equação de Van der Pol e um Canard.

Um fenômeno inesperado pode ser observado neste exemplo. Existe a possibilidade de certos Canards conterem trechos da curva lenta $L$ formados por pontos normalmente hiperbólicos repulsores (ver definição na continuação). Tal fato parece pouco provável, pois próximo a tais pontos existe uma repulsão exponencialmente forte que deveria empurrar quase todos os 
pontos para longe de $L$. O artigo [WE] fornece uma explicação para tal fenômeno utilizando análise assintótica clássica.

Em nosso caso, o Fenômeno Canard será olhado como um problema de intersecção de variedades Centrais do tipo atrator-repulsor, chamadas de Superfícies Canard associadas a uma família analítica de campo de vetores.

Consideremos o campo de vetores analítico

$$
X_{\varepsilon, a}=\varepsilon(\partial / \partial x)+F(x, y, \varepsilon, a)(\partial / \partial y), \quad \varepsilon \in \mathbb{R}, a \in\left(\mathbb{R}^{n}, 0\right),
$$

sobre um aberto $U_{x} \times U_{y} \subset \mathbb{R}^{2}$, tal que $F(x, y, 0,0)=y f(x, y)$, onde $f(x, y)$ é uma função analítica não divisível por $y$.

Definição 1.0.1 Seja $\gamma$ uma curva no espaço de parâmetros $\mathbb{R}^{+} \times \mathbb{R}^{n}$

$$
\begin{aligned}
\gamma:[0, \delta) & \longrightarrow \mathbb{R}^{+} \times \mathbb{R}^{n}, \quad(\delta>0) \\
\rho & \longmapsto \gamma(\rho)=(\varepsilon(\rho), a(\rho)),
\end{aligned}
$$

chamada de curva de controle, satisfazendo

- $\gamma(0)=0$,

- $\varepsilon(\rho)>0$ se $\rho>0$.

Uma Superfície Canard para $X_{\varepsilon, a}$ é uma variedade contínua 2-dimensional

$$
W^{\gamma}=\operatorname{graf}\{y=w(x, \rho)\} \subset M, \quad \rho \geq 0
$$

localmente invariante pela família a um parâmetro de campos de vetores

$X_{\rho}^{\gamma}=X_{\varepsilon(\rho), a(\rho)}=\varepsilon(\rho)(\partial / \partial x)+F(x, y, \varepsilon(\rho), a(\rho))(\partial / \partial y), \quad \rho \in\left(\mathbb{R}^{+}, 0\right)$,

definida sobre a subvariedade 3-dimensional $M=U_{x} \times U_{y} \times\left(\mathbb{R}^{+}, 0\right)$, e tal que $W^{\gamma} \cap\{\rho=0\} \subset L=M \cap \operatorname{Sing}\left(X_{0,0}\right)$.

Note que o conjunto de singularidades para o campo restrito $X_{\rho}^{\gamma}$ é dado pela curva $\Gamma=\{\rho=y=0\} \subset L$.

Como a existência de estas Superfícies Canard não está sempre garantida, podemos colocar o seguinte problema:

Problema Canard Local (PCL):

Dada uma familia analítica de campos de vetores $X_{\varepsilon, a}$ como acima, definida sobre $U_{x} \times U_{y} \subset \mathbb{R}^{2}$ podemos sempre encontrar uma curva de controle $\rho \longmapsto \gamma(\rho)$ no espaço de parâmetros tal que a familia restrita $X_{\rho}^{\gamma}=X_{(\varepsilon(\rho), a(\rho))}$ tenha uma superficie Canard $W^{\gamma}$ ?.

Se o conjunto de singularidades $\operatorname{Sing}\left(X_{0,0}\right)$ não é normalmente hiperbólico, a resposta para o problema PCL pode ser negativa; como mostra o seguinte exemplo. 
Exemplo 1.0.2 Consideremos a família

$$
X_{\varepsilon}=\varepsilon(\partial / \partial x)+(x y+\varepsilon)(\partial / \partial y), \quad \varepsilon \in \mathbb{R}^{+} .
$$

A curva $\Gamma=\{y=\varepsilon=0\}$ é uma curva de singularidades normalmente hiperbólicas exceto em $x=0$. De fato, temos que

$$
\operatorname{DX}_{0}(x, 0)=\left[\begin{array}{ll}
0 & 0 \\
0 & x
\end{array}\right]
$$

Logo, $(x, 0)$ é atrator se $x<0$, ou repulsor se $x>0$, ou seja $\Gamma \cap\{x<0\}$ é o conjunto de singularidades normalmente hiperbólicas atratoras e $\Gamma \cap\{x>0\}$ de repulsoras. A repulsão exponencial que existe para $x>0$, impede a existência de Superfícies Canards.

De fato suponha que $y=w(x, \varepsilon)$ define uma Superfície Canard onde $w(x, \varepsilon)$ é uma função contínua definida em um domínio da forma $x \in U_{x}=$ $\left(-x_{0}, x_{0}\right), x_{0}>0 \quad \varepsilon \in U_{\varepsilon}=\left[0, \varepsilon_{0}\right)$. Para $\varepsilon>0$ podemos escrever

$$
w(x, \varepsilon)=e^{\frac{x^{2}}{2 \varepsilon}}\left(w\left(-x_{0}, \varepsilon\right) e^{\frac{-1}{2 \varepsilon}}+\int_{-x_{0}}^{x} e^{\frac{-t^{2}}{2 \varepsilon}} d t\right),
$$

pois $w(x, \varepsilon)$ é solução do problema de Cauchy

$$
\begin{cases}\frac{d}{d x} y & =\frac{x}{\varepsilon} y+1 \\ y\left(-x_{0}\right) & =w\left(-x_{0}, \varepsilon\right)\end{cases}
$$

Suponhamos que $w(x, \varepsilon)$ é uma função contínua sobre qualquer vizinhança da origem contida em $U_{x} \times U_{\varepsilon}$, tal que $w(x, 0)=0$.

Considerando a curva $\varepsilon=x^{3}$, para $0 \leq x \leq x_{0}$, a função $h(x)=w\left(x, x^{3}\right)$ dada por

$$
h(x)=w\left(-x_{0}, \varepsilon\right) e^{\frac{1}{2 x}-\frac{1}{2 x^{3}}}+e^{\frac{1}{2 x}} \int_{-x_{0}}^{x} e^{\frac{-t^{2}}{2 x^{3}}} d t
$$

também deve ser contínua na origem. Note que para $t \in\left[\frac{x}{3}, \frac{x}{2}\right]$, vale a relação

$$
\frac{-1}{8 x} \leq \frac{-t^{2}}{2 x^{3}} \leq \frac{-1}{18 x}
$$

Assim o segundo termo da função h satisfaz à desigualdade

$$
e^{\frac{1}{2 x}} \int_{-x_{0}}^{x} e^{\frac{-t^{2}}{2 x^{3}}} d t \geq e^{\frac{1}{2 x}}\left(\frac{1}{6} x e^{\frac{-1}{3 x}}\right)
$$

Portanto, quando $x \longrightarrow 0$, claramente $h(x) \longrightarrow \infty$. Absurdo. 
Na família $X_{\varepsilon, a}$, escrevamos para a função $F=F(x, y, \varepsilon, a)$ a seguinte expansão em série de potências.

$$
\begin{aligned}
F= & \left(\sum_{i=0}^{2 p-1} A_{i}(\varepsilon, a) x^{i}+x^{2 p}\left(\sum_{i=1}^{\infty} A_{2 p+i-1}(\varepsilon, a) x^{i-1}\right)\right) \\
& +\left(\sum_{j=0}^{p-1} B_{j}(\varepsilon, a) x^{j}+B_{p}(\varepsilon, a) x^{p}+x^{p}\left(\sum_{j=2}^{\infty} B_{p+i-1}(\varepsilon, a) x^{j-1}\right)\right) y \\
& +Q(x, y, \varepsilon,) y^{2} .
\end{aligned}
$$

e consideremos sobre a singularidade degenerada $x=0 \in \Gamma$ a seguinte hipótese:

Hipótese de Transversalidade (HT):

Temos $B_{p}(0,0) \neq 0$ e a função analítica

$$
\begin{aligned}
\varphi: & \mathbb{R}^{n} \longrightarrow \mathbb{R}^{3 p} \\
& a \longmapsto\left(A_{0}(0, a), \ldots, A_{2 p-1}(0, a), B_{0}(0, a), \ldots, B_{p-1}(0, a)\right),
\end{aligned}
$$

é uma submersão numa vizinhança de $a=0$.

O resultado principal deste trabalho é o seguinte:

Teorema 1.0.3 Se a familia $X_{\varepsilon, a}$, satisfaz a hipótese (HT) na singularidade degenerada $x=0 \in \Gamma$, então o problema (PCL) tem resposta positiva.

Os passos da demonstração são os seguintes:

1. No capítulo 3 , vamos construir variedades centrais dinâmicas $W$, invariantes pela família de campos no plano

$$
X_{\varepsilon, a}=\varepsilon(\partial / \partial x)+F(x, y, \varepsilon, a)(\partial / \partial y), \quad(\varepsilon, a) \in \mathbb{R}^{+} \times\left(\mathbb{R}^{n}, 0\right),
$$

onde $\left.a=\left((A, B), \mathcal{A}_{r}\right)\right) \in\left(\mathbb{R}^{3 p} \times \mathbb{R}^{n-3 p}, 0\right)$, definidas sobre conjuntos normalmente hiperbólicos $\Gamma \backslash\{0\}$.

2. No capítulo 4, será feita a desingularização da singularidade degenerada

$$
x=0 \in \Gamma \in(\mathbb{R}, 0)
$$

pela aplicação Blowing-up $\Phi$ dada no capítulo 2.

3. Serão construídas, no capítulo 6 , variedades centrais dinâmicas $W^{ \pm}$ sobre o conjunto invariante (divisor excepcional)

$$
\left.\Phi^{-1}(0)\right|_{\left\{\varepsilon=\mathcal{A}_{r}=0\right\}}, \quad\left(\varepsilon, \mathcal{A}_{r}\right) \in \mathbb{R}^{+} \times\left(\mathbb{R}^{n-3 p}, 0\right),
$$

com propriedades assintóticas dadas no capítulo 5 . 
4. Determinamos no capítulo 7, a Região Canard

$$
\mathcal{O}_{\tilde{\Gamma}} \subset\left(\mathbb{R}^{+} \times \mathbb{R}^{n}, 0\right), \quad \tilde{\Gamma} \subset \Gamma,
$$

e conseqüentemente apresentamos a Superfície Canard representada pelo par $\left(\mathcal{O}_{\tilde{\Gamma}}, w\right)$, em termos das aplicações blowing-up $\Phi$ e Blowingdown $\Phi^{-1}$. 


\section{Capítulo 2}

\section{Conceitos Preliminares}

\subsection{Funções Especiais}

Com a finalidade de explicar a dinâmica de campos de vetores próxima a singularidades degeneradas, introduzimos uma mudança singular de variáveis, denominada blowing-up.

\subsubsection{Aplicação Blowing-up}

Dado $k \in \mathbb{N}$, seja $X=\sum_{i=1}^{n} X_{i} \frac{\partial}{\partial x_{i}}$ um campo de vetores de classe $C^{k}$ sobre $\mathbb{R}^{n}$, tal que $X(0)=0$. Então consideremos a seguinte coleção de campos de vetores $\left\{V, V_{i, j}\right\}$, onde

(a) $V_{i, j}=\frac{1}{2}\left(x_{i} \frac{\partial}{\partial x_{j}}-x_{j} \frac{\partial}{\partial x_{i}}\right)$, é um campo vetorial sobre $\mathbb{R}^{n}$, que satisfaz às seguintes fórmulas:

- Para $n=2$

$$
\begin{aligned}
\sum_{i, j=1}^{2}<X, V_{i j}>\cdot V_{i j} & =<X, V_{12}>\cdot V_{12}+<X, V_{21}>\cdot V_{21} \\
& =2<X, V_{12}>\cdot V_{12}
\end{aligned}
$$


- Para $n=3$

$$
\begin{aligned}
\sum_{i, j=1}^{3}<X, V_{i j}>. V_{i j}= & <X, V_{12}>\cdot V_{12}+<X, V_{13}>\cdot V_{13} \\
& +<X, V_{21}>\cdot V_{21}+<X, V_{23}>\cdot V_{23} \\
& +<X, V_{31}>. V_{31}+<X, V_{32}>\cdot V_{32} \\
= & 2<X, V_{12}>. V_{12}+2<X, V_{13}>. V_{13} \\
& +2<X, V_{23}>. V_{23}
\end{aligned}
$$

- No caso geral, obtemos a seguinte expressão:

$$
\begin{aligned}
\sum_{i, j=1}^{n}<X, V_{i j}>. V_{i j}= & 2 \sum_{j=2}^{n}<X, V_{1 j}>\cdot V_{1 j}+ \\
& +2 \sum_{j=3}^{n}<X, V_{2 j}>\cdot V_{2 j}+ \\
& +2 \sum_{j=4}^{n}<X, V_{3 j}>. V_{3 j}+ \\
& +\ldots+2<X, V_{(n-1) n}>\cdot V_{(n-1) n}
\end{aligned}
$$

(b) $V=\sum_{i=1}^{n} x_{i} \frac{\partial}{\partial x_{i}}$, é um campo vetorial sobre $\mathbb{R}^{n}$, que satisfaz o seguinte:

- $|V|^{2}=x_{1}^{2}+\ldots+x_{n}^{2}$

- Para $n=2$, notemos que $<X, V_{12}>=-\frac{1}{2}\left(x_{2} X_{1}-x_{1} X_{2}\right)$, logo

$$
\begin{aligned}
<X, V>\cdot V & =|V|^{2} \cdot X+\left(x_{2} X_{1}-x_{1} X_{2}\right) \cdot V_{12} \\
& =|V|^{2} \cdot X-2<X, V_{12}>\cdot V_{12}
\end{aligned}
$$

- Para $n=3$, analogamente obtemos:

$$
\begin{aligned}
<X, V>\cdot V= & |V|^{2} \cdot X+\left(x_{2} X_{1}-x_{1} X_{2}\right) \cdot V_{12}+ \\
& +\left(x_{3} X_{1}-x_{1} X_{3}\right) \cdot V_{13}+\left(x_{3} X_{2}-x_{2} X_{3}\right) \cdot V_{23} \\
= & |V|^{2} \cdot X-2<X, V_{12}>\cdot V_{12}- \\
& -2<X, V_{13}>\cdot V_{13}-2<X, V_{23}>\cdot V_{23}
\end{aligned}
$$

De modo geral, para $n \in \mathbb{N}$ arbitrário obtemos uma expressão para o campo $X$ na base $\left\{V, V_{i, j}\right\}$, dada pela igualdade

$$
<X, V>. V+\sum_{i<j}^{n}<X, V_{i j}>\cdot V_{i j}=|V|^{2} \cdot X
$$


Definamos a aplicação

$$
\begin{aligned}
\Phi: \quad & \mathbb{S}^{n-1} \times \mathbb{R} \longrightarrow \mathbb{R}^{n} \\
& (\bar{x}, r)=\left(\bar{x}_{1}, \ldots, \bar{x}_{n}, r\right) \longrightarrow\left(r \bar{x}_{1}, \ldots, r \bar{x}_{1}\right)=r \bar{x}=x
\end{aligned}
$$

Onde, $\quad \mathbb{S}^{n-1}=\left\{\left(\bar{x}_{1}, \ldots, \bar{x}_{n}\right) \mid \sum_{i=1}^{n} \bar{x}_{i}^{2}=1\right\}$. Note que $\Phi\left(\mathbb{S}^{n-1} \times\{0\}\right)=0$. Esta aplicação faz uma correspondência difeomorfa entre os conjuntos $\{r \neq$ $0\}$ e $\mathbb{R}^{n} \backslash\{0\}$

Observação 2.1.1 Para o caso $n=2$, a aplicação $\Phi$ corresponde á transformação em coordenadas polares habitual.

$$
(\alpha, r) \longrightarrow(r \cos (\alpha), r \sin (\alpha))
$$

Note que a coleção de campos de vetores $\left\{X, V, V_{i, j}\right\}$ dados acima, nas coordenadas da aplicação Blowing-up $\Phi$ satisfaz às seguintes fórmulas:

(1)

$$
\begin{aligned}
<X, V>(\Phi(\bar{x}, r)) & =\sum_{i, j=1}^{n} X_{i} x_{j} \delta_{i j}(\Phi(\bar{x}, r)) \\
& =r \sum_{i=1}^{n} \bar{x}_{i} X_{i}(\Phi(\bar{x}, r))
\end{aligned}
$$

(2)

$$
\begin{aligned}
<X, V_{i j}>(\Phi(\bar{x}, r)) & =\frac{1}{2}\left[\sum_{k=1}^{n} X_{k} x_{i} \delta_{k j}-\sum_{k=1}^{n} X_{k} x_{j} \delta_{k i}\right](\Phi(\bar{x}, r)) \\
& =\frac{1}{2}\left[x_{i} X_{j}-x_{j} X_{i}\right](\Phi(\bar{x}, r)) \\
& =\frac{r}{2}\left[\bar{x}_{i} X_{j}(\Phi(\bar{x}, r))-\bar{x}_{j} X_{i}(\Phi(\bar{x}, r))\right]
\end{aligned}
$$

(3) $|V|^{2}=r^{2}$

Proposição 2.1.2 Seja $X$ um campo vetorial de classe $C^{k}$ sobre $\mathbb{R}^{n}$ : com $X(0)=0$. Então existe um campo vetorial $\tilde{X}$ de classe $C^{k-1}$ sobre $\mathbb{S}^{n-1} \times$ $\mathbb{R}^{+}$, tal que, $\Phi_{*}(\widetilde{X})=X,(\Phi$ é o Blowing-up considerado $)$. 


\section{DEMONSTRAÇÃo}

Consideremos as funções de classe $C^{k}$

$$
\alpha_{r}=<X, V>: \mathbb{R}^{n} \longrightarrow \mathbb{R}, \quad \alpha_{i j}=<X, V_{i j}>: \mathbb{R}^{n} \longrightarrow \mathbb{R}
$$

Onde

$$
V=\sum_{i=1}^{n} x_{i} \frac{\partial}{\partial x_{i}}, \quad V_{i, j}=\frac{1}{2}\left(x_{i} \frac{\partial}{\partial x_{j}}-x_{j} \frac{\partial}{\partial x_{i}}\right)
$$

E definamos os campos vetoriais $\widetilde{V}$ e $\widetilde{V}_{i j}$, como sendo

$$
\Phi_{*}(\tilde{V})=V, \quad \Phi_{*}\left(\widetilde{V}_{i j}\right)=V_{i j}
$$

Realmente, verifica-se que $\widetilde{V}=r \frac{\partial}{\partial r}$, e $\widetilde{V}_{i j}$ são rotações em $\mathbb{S}^{n-1}$, sobre o plano $\left(\bar{x}_{i}, \bar{x}_{j}\right)$. Pelas observações anteriores:

$$
\Phi_{*}\left(\frac{1}{r^{2}}\left\{\alpha_{r}(\Phi) \tilde{V}+\sum_{i, j=1}^{n} \alpha_{i j}(\Phi) \widetilde{V_{i j}}\right\}\right)=X
$$

Onde, $\quad \alpha_{r}(\Phi)$ e $\alpha_{i j}(\Phi)$ são funções de classe $C^{k}$, sobre $\mathbb{S}^{n-1} \times \mathbb{R}^{+}$, as quais se anulam no conjunto $\{r=0\}$, pois

$$
X(0)=0, V(0)=0, V_{i j}(0)=0
$$

E como

$$
\begin{aligned}
\frac{1}{r^{2}} \alpha_{r}(\Phi) & =\sum_{i=1}^{n} \bar{x}_{i} \frac{1}{r} X_{i}(\Phi) \\
\frac{1}{r^{2}} \alpha_{i j}(\Phi) & =\frac{1}{2}\left[\bar{x}_{i} \frac{1}{r} X_{j}(\Phi)-\bar{x}_{j} \frac{1}{r} X_{i}(\Phi)\right]
\end{aligned}
$$

são funções de classe $C^{k-1}$, sobre $\{r \neq 0\}$; então temos que

$$
\tilde{X}=\frac{1}{r^{2}}\left\{\alpha_{r}(\Phi) \widetilde{V}+\sum_{i, j=1}^{n} \alpha_{i j}(\Phi) \widetilde{V}_{i j}\right\}
$$

é de fato um campo vetorial de classe $C^{k}$ sobre $\left(\mathbb{S}^{n-1} \times \mathbb{R}^{+}\right) \backslash\{r=0\}$; que se estende diferenciavelmente em classe $C^{k-1}$ a $D=\{r=0\}$. 
Definição 2.1.3 Definamos o Blowing-up quasi-homogêneo, como sendo uma aplicação analítica definida como

$$
\begin{aligned}
\Phi: \mathbb{S}^{n-1} \times \mathbb{R}^{+} & \longrightarrow \mathbb{R}^{n} \\
(\bar{x}, \tau):=\left(\bar{x}_{1}, \bar{x}_{2}, \ldots, \bar{x}_{n}, \tau\right) & \longmapsto\left(\tau^{\alpha_{1}} \bar{x}_{1}, \tau^{\alpha_{2}} \bar{x}_{2}, \ldots, \tau^{\alpha_{n}} \bar{x}_{n}\right):=\tau^{\alpha} \cdot x
\end{aligned}
$$

onde $\alpha=\left(\alpha_{1}, \alpha_{2}, \ldots, \alpha_{n}\right) \in\left(\mathbb{Z}^{+}\right)^{n}$ é o vetor de pesos e $\mathbb{S}^{k-1}=\left\{\left(z_{1}, \ldots, z_{k}\right) \in\right.$ $\left.\mathbb{R}^{k} \mid z_{1}^{2}+\ldots+z_{k}^{2}=1\right\}$.

Note que quando $\alpha=(1,1, \ldots, 1)$, temos o Blowing-up em coordenadas polares. Um resultado análogo à proposição 2.1 .2 pode ser provado para blowing-up quasi-homogêneos.

Observação 2.1.4 Para campos $X$ tais que $J^{k}(X)(0)=0, e \quad J^{k+1}(X)(0) \neq$ 0 , para algum $k \geq 0$, o campo

$$
\bar{X}=\frac{1}{r^{k}} \tilde{X}
$$

é diferenciável e não se anula identicamente em $D=\{r=0\}$; neste caso diremos que a singularidade $0 \in \mathbb{R}^{n}$ é de multiplicidade $k$, e que $\bar{X}$ é o transformado estrito de $X$.

\subsubsection{Extensões Blowing-up $C^{k}$}

Seja $M \subset \mathbb{R}^{n}$ um subconjunto fechado, denotemos simplesmente por $C^{k}(M)$ o espaço vetorial de funções de classe $C^{k}$ (no sentido de Whitney) sobre $M$ (ver $[\mathrm{M}])$.

Definição 2.1.5 Dado um subconjunto aberto $U \subset \mathbb{R}^{n}$ dizemos que $f \in$ $C^{k}(U)$ tem extensão- $C^{k}$ a $\bar{U}$ se $f \in C^{k}(\bar{U})$. Equivalentemente $f \in C^{k}(U)$ tem extensão $C^{k}$ a $\bar{U}$ se para todo $x \in \bar{U}$ existe uma vizinhança $V=V(x)$ de $x$ tal que $f \in C^{k}(\overline{U \cap V})$.

Seja $U \subset \mathbb{R}^{n}$ um subconjunto aberto e consideremos a decomposição $\mathbb{R}^{n}=\mathbb{R}^{m} \times \mathbb{R}^{n-m}$ tal que cada ponto de $\mathbb{R}^{n}$ tenha coordenadas $(x, y)$.

Definição 2.1.6 Diremos que uma função $f \in C^{k}(U)$, tem extensão blowingup $C^{k}$ em $\{x=0\}$, se existe um multi-indice $\alpha=\left(\alpha_{1}, \ldots, \alpha_{m}\right)$, tal que, ao considerar a aplicação de Blowing-up

$$
\begin{aligned}
\phi: & \mathbb{S}^{m-1} \times \mathbb{R}^{+} \times \mathbb{R}^{n-m} \longrightarrow \mathbb{R}^{n} \\
& (\bar{x}, \tau, y) \longmapsto\left(\tau^{\alpha_{1}} \bar{x}_{1}, \ldots, \tau^{\alpha_{m}} \bar{x}_{m}, y\right):=\left(\tau^{\alpha} \bar{x}, y\right)
\end{aligned}
$$

a função $F=f \circ \phi: \phi^{-1}(U)=\mathcal{U} \longrightarrow U$ tem extensão $C^{k}$ a $\overline{\mathcal{U}}$, (ou seja $f \circ \phi \in C^{k}(\overline{\mathcal{U}})$ no sentido de Whitney). 
Observação 2.1.7 Este tipo de funções nos permitem estudar localmente os pontos nos quais a diferenciabilidade é perdida. Veremos que as superfícies Canard estudadas adiante pertencem a tais classes.

Exemplo 2.1.8 No aberto $U=\mathbb{R}^{2} \backslash\{0\}$, consideremos a função

$$
f\left(x_{1}, x_{2}\right)=\sqrt{x_{1}^{2}+x_{2}^{2}}, \quad \in C^{\infty}(U)
$$

note que $f \notin C^{\infty}(\bar{U})$. Consideremos a aplicação blowing-up

$$
\phi: \begin{gathered}
\mathbb{S}^{1} \times \mathbb{R}^{+} \longrightarrow \mathbb{R}^{2} \\
\left(\bar{x}_{1}, \bar{x}_{2}, \tau\right) \longmapsto\left(\tau \bar{x}_{1}, \tau \bar{x}_{2}\right)
\end{gathered}
$$

claramente temos $F=f \circ \phi\left(\bar{x}_{1}, \overline{x_{2}}, \tau\right)=\tau$.

Note que $\mathbb{S}^{1}=\{\tau=0\}$; se consideramos o aberto

$$
\mathcal{U}=\phi^{-1}(U)=\left\{\left(\bar{x}_{1}, \bar{x}_{2}, \tau\right) \mid \tau>0\right\}=\{\tau>0\}
$$

então como $F(\bar{x}, \tau)=\tau \in C^{\infty}(\mathcal{U})$ temos que $F \in C^{\infty}(\overline{\mathcal{U}})=C^{\infty}(\tau \geq 0)$. Portanto, $f$ tem extensão blowing-up $C^{k}$ em $\{x=0\}$.

Lema 2.1.9 Suponha que $f$ tem extensão blowing-up $C^{k}$ em $\{x=0\}$ e seja $\gamma \in C^{\infty}\left([0, \delta), \mathbb{R}^{n}\right)$ uma curva $(\delta>0)$ tal que $\gamma((0, \delta)) \subset U \backslash\{x=0\}$. Então existe $p \in \mathbb{N} \backslash\{0\}$ tal que $f \circ \gamma=: \tilde{f} \in C^{k}\left(\left[0, \delta^{\frac{1}{p}}\right)\right.$ ), (onde $\tilde{f}(w)=f \circ \gamma\left(w^{p}\right)$ )

\section{DEMONSTRAÇÃo}

Seja $I=(0, \delta), I_{p}=\left(0, \delta^{\frac{1}{p}}\right), \mathcal{U}=\phi^{-1}(U)$. Usando a expressão do blowing-up $\phi$, é fácil ver que existe um número natural $p \in \mathbb{N} \backslash\{0\}$ e uma curva $\tilde{\gamma} \in C^{\infty}\left(I_{p}, \mathbb{S}^{m-1} \times \mathbb{R}^{+} \times \mathbb{R}^{n-m}\right)$ tal que $\gamma \circ \varphi=\phi \circ \tilde{\gamma}$, onde $\varphi(w)=w^{p}$, $w \in I_{p}, w^{p} \in I$. Por hipótese $F=f \circ \phi \in C^{k}(\overline{\mathcal{U}})$; da relação

$$
\tilde{f}(w):=f \circ \gamma\left(w^{p}\right)=f \circ \gamma \circ \varphi(w)=f \circ \phi \circ \tilde{\gamma}(w)=F \circ \tilde{\gamma}(w)
$$

segue $\tilde{f}=F \circ \tilde{\gamma} \in C^{k}\left(\bar{I}_{p}\right)$.

\subsubsection{Funções $\infty$-Flat}

Definição 2.1.10 Sejam $M \subset \mathbb{R}^{n}$ um subconjunto fechado, e $U \subset \mathbb{R}^{n}$ um subconjunto aberto. Dizemos que $f \in C^{k}(U)$ é $\infty$ - flat a $M$, denotado por $f \in C_{\text {flat }}^{k}(U, M)$, se para cada subconjunto compacto $K \subset \mathbb{R}^{n}$ tal que

$$
K \backslash M \subset U
$$


e para cada $m \in \mathbb{N}$, existe uma constante $C=C(K, m)>0$ tal que

$$
|f(x)| \leq C(d(x, M \cap K))^{m}, \quad \forall x \in K .
$$

onde d é uma métrica em $\mathbb{R}^{n}$.

Em particular se $M=\{0\}, f \in C_{\text {flat }}^{k}(U, 0)$; para todo subconjunto compacto $K \subset \mathbb{R}^{n}$ com $K \backslash\{0\} \subset U$, existem constantes $m \in \mathbb{N}$ e $C=C(K, m)>0$ tal que

$$
|f(x)| \leq C(|x|)^{m}, \quad \forall x \in K .
$$

Exemplo 2.1.11 Vejamos os seguintes exemplos:

1. $f\left(x_{1}, x_{2}\right)=F\left(x_{1}\right) \exp \left(\frac{-1}{\left|x_{2}\right|}\right) \in C_{f l a t}^{k}\left(U,\left\{x_{2}=0\right\}\right)$, onde $F \in C^{k}(\mathbb{R})$ é uma função arbitrária e $U=\mathbb{R}^{2} \backslash\left\{x_{2}=0\right\}$.

Com efeito, como $\exp \left(\frac{1}{\left|x_{2}\right|}\right)>\frac{1}{\left|x_{2}\right|}$, e F sendo limitada em todo compacto $K \subset \mathbb{R}^{2} \backslash\left\{x_{2}=0\right\}$, existe uma constante $C=C(K)>0$, que depende de $K$, tal que

$$
\left|f\left(x_{1}, x_{2}\right)\right| \leq C\left|x_{2}\right|, \quad \forall\left(x_{1}, x_{2}\right) \in K .
$$

2. $f(x)=\exp \left(\frac{-1}{\|x\|^{\alpha}}\right) \in C_{\text {flat }}^{\infty}(U,\{0\})$, onde $U=\mathbb{R}^{n} \backslash\{0\}$.

Com efeito, como $\exp \left(\frac{1}{\|x\|^{\alpha}}\right)>\frac{1}{\|x\|^{\alpha}}$, é imediato ver que

$$
|f(x)| \leq\|x\|^{\alpha}, \forall \alpha \in \mathbb{R}^{+} \backslash\{0\} .
$$

3. $f(x, y)=|y| \exp \left(\frac{-1}{x}\right) \in C_{\text {flat }}^{0}(U,\{0\})$, onde $U=\mathbb{R}^{2} \cap\{x>0\}$.

De fato para cada $(x, y) \in K$ com a norma da soma em $\mathbb{R}^{2}$, temos

$$
|f(x, y)| \leq|y||x| \leq|x|^{2}+2|x||y|+|y|^{2}=(|x|+|y|)^{2}=|(x, y)|^{2} .
$$

Definição 2.1.12 Seja $M \subset \mathbb{R}^{n}$ um subconjunto fechado, e $U \subset \mathbb{R}^{n}$ um subconjunto aberto. Para $\hat{f} \in C^{\infty}(M)$ considere a extensão Whitney $f=$ $E(\hat{f}) \in C^{\infty}\left(\mathbb{R}^{n}\right)$. Diremos que $g \in C^{\infty}(U)$ é $\infty$-flat a $\hat{f}$ denotado por $g \in C_{\text {flat }}^{\infty}(U, M, \hat{f})$ se $(g-f) \in C_{\text {flat }}^{\infty}(U, M)$.

\subsubsection{Blow-down $\infty$-Flat}

Consideremos a aplicação blowing-up

$$
\begin{aligned}
\phi: & \mathbb{S}^{n-1} \times \mathbb{R}^{+} \longrightarrow \mathbb{R}^{n} \\
& \left(\bar{x}_{1}, \ldots, \bar{x}_{n}, \tau\right) \longmapsto\left(\tau^{\alpha_{1}} \bar{x}_{1}, \ldots, \tau^{\alpha_{n}} \bar{x}_{n}\right)
\end{aligned}
$$


onde $\alpha=\left(\alpha_{1}, \ldots, \alpha_{n}\right) \in \mathbb{N}^{n} \backslash\{0\}$. Utilizaremos as notações $(\bar{x}, \tau)=\left(\bar{x}_{1}, \ldots, \bar{x}_{n}, \tau\right)$, e $\tau^{\alpha} \bar{x}:=\left(\tau^{\alpha_{1}} \bar{x}_{1}, \ldots, \tau^{\alpha_{n}} \bar{x}_{n}\right)$.

Seja $F \in C^{k}(U)$ uma função arbitrária definida sobre o conjunto aberto $U=\left\{(\bar{x}, \tau) \in \mathbb{S}^{n-1} \times \mathbb{R}^{+} \mid \tau>0\right\}$, e $D=\{\tau=0\} \subset \mathbb{S}^{n-1}$ o divisor excepcional. Sendo $\phi$ um difeomorfismo em $\mathbb{S}^{n-1} \times \mathbb{R}^{+} \cap\{\tau>0\}$, definamos sobre $\mathcal{U}=\phi(U) \subset \mathbb{R}^{n}$ a função

$$
\mathcal{F}(x):=F \circ \phi^{-1}(x)
$$

de onde $\mathcal{F} \in C^{k}(\mathcal{U})$.

Lema 2.1.13 $F \in C_{\text {flat }}^{\infty}(U, D)$ se e só se $\mathcal{F} \in C_{\text {flat }}^{\infty}(\mathcal{U}, 0)$

\section{DEMONSTRAÇÃO}

i) (Condição Necessária)

Suponha que $F \in C_{\text {flat }}^{\infty}(U, D)$, então por definição, para cada conjunto compacto $K \subset \mathbb{S}^{n-1} \times \mathbb{R}^{+}$, tal que $K \backslash D \subset U$, e cada $n \in \mathbb{N}$, existe uma constante $C=C(K, n)>0$ tal que

$$
|F(\bar{x}, \tau)| \leq C(d((\bar{x}, \tau), K \cap D))^{n}, \quad \forall(\bar{x}, \tau) \in K .
$$

Logo como $d((\bar{x}, \tau), K \cap D)=d(\tau, 0)$, temos

$$
|F(\bar{x}, \tau)| \leq C|\tau|^{n}, \quad \forall \tau>0
$$

consideramos agora um subconjunto compacto $\mathcal{K} \subset \mathbb{R}^{n}$, tal que

$$
\mathcal{K} \backslash\{0\} \subset \mathcal{U}=\phi(U) .
$$

Logo existe um subconjunto compacto $K \subset \mathbb{S}^{n-1} \times \mathbb{R}^{+}$, tal que

$$
\mathcal{K}=\phi(K), \quad K \backslash D \subset U,
$$

assim para cada $x \in \mathcal{K}$, existe $(\bar{x}, \tau) \in K$, tal que $x=\phi(\bar{x}, \tau):=\tau^{\alpha} \bar{x}$. Observemos dois casos

Se $0 \leq \tau<1$, considere $a=\max \left\{\alpha_{1}, \ldots, \alpha_{n}\right\}$, para obter

$$
\begin{aligned}
|\tau|^{a} & =\left|\tau^{a}\right|\|\bar{x}\|=\left\|\tau^{a} \bar{x}\right\| \\
& =\left\|\left(\tau^{a} \bar{x}_{1}, \ldots, \tau^{a} \bar{x}_{n}\right)\right\| \\
& \leq\left\|\left(\tau^{\alpha_{1}} \bar{x}_{1}, \ldots, \tau^{\alpha_{n}} \bar{x}_{n}\right)\right\| \\
& =\left\|\tau^{\alpha} \bar{x}\right\|=\|x\| .
\end{aligned}
$$


Se $\tau \geq 1$, por compacidade existem constantes $B=B(\mathcal{K})>0, C=$ $C(\mathcal{K})>0$ tal que $|\tau|^{a} \leq B$ e $C \leq\|x\|$, de onde:

$$
|\tau|^{a} \leq \frac{B}{C}\|x\|, \quad \forall x \in \mathcal{K} .
$$

Assim resulta

$$
\begin{aligned}
|\mathcal{F}(x)| & =\left|F \circ \phi^{-1}(x)\right|=|F(\bar{x}, \tau)| \\
& \leq C(K, n)|\tau|^{n} \leq C(K, n)|\tau|^{n a} \\
& \leq\left. C(K, n) C_{1}(\mathcal{K})^{n}|| x\right|^{n},
\end{aligned}
$$

onde, $C_{1}(\mathcal{K})=\max \left\{1, \frac{B}{C}\right\}$.

Definindo a constante $\mathcal{C}(\mathcal{K}, n)=C(K, n) C_{1}(\mathcal{K})^{n}>0$, obtemos

$$
|\mathcal{F}(x)| \leq \mathcal{C}(\mathcal{K}, n)\|x\|^{n}, \quad \forall x \in \mathcal{K} \subset \mathcal{U} .
$$

Portanto $\mathcal{F} \in C_{\text {flat }}^{\infty}(\mathcal{U}, 0)$.

ii) (Condição Suficiente)

Em forma análoga, observemos os dois casos

Se $0 \leq \tau<1$

$$
\begin{aligned}
\left\|\tau^{\alpha} \bar{x}\right\| & =\left\|\left(\tau^{\alpha_{1}} \bar{x}_{1}, \ldots, \tau^{\alpha_{n}} \bar{x}_{n}\right)\right\| \\
& \leq\left|\tau^{a}\right||| \bar{x} \|=|\tau|^{a},
\end{aligned}
$$

onde, $a=\inf \left\{\alpha_{1}, \ldots, \alpha_{n}\right\}$.

Se $\tau \geq 1$, é claro que $|\tau|^{a} \geq 1$, e por compacidade existe uma constante $C_{2}=C_{2}(K)>0$ tal que $\left\|\tau^{\alpha} \bar{x}\right\| \leq C_{2}$; assim temos

$$
\left\|\tau^{\alpha} \bar{x}\right\| \leq C_{2}|\tau|^{a}, \quad \forall(\bar{x}, \tau) \in K .
$$

Logo supondo válido

$$
|\mathcal{F}(x)| \leq \mathcal{C}(\mathcal{K}, m)\|x\|^{m}, \quad \forall x \in \mathcal{K} \subset \mathcal{U} .
$$

Consideremos $K=\phi^{-1}(\mathcal{K})$, tal que $K \backslash D \subset U=\phi^{-1}(\mathcal{U})$, sendo $\mathcal{F}=F \circ \phi^{-1}$, temos

$$
\begin{aligned}
|F(\bar{x}, \tau)| & =|\mathcal{F} \circ \phi(\bar{x}, \tau)| \\
& \leq \mathcal{C}(\mathcal{K}, m) \|\left.\tau^{\alpha} \bar{x}\right|^{m} \\
& \leq \mathcal{C}(\mathcal{K}, m) C_{2}(K)^{m}|\tau|^{a m}
\end{aligned}
$$


Definindo, $C(K, m)=\mathcal{C}(\mathcal{K}, m) C_{2}(K)^{m}$, temos que

$$
|F(\bar{x}, \tau)| \leq C(K, m)|\tau|^{a m}
$$

e como $d(\tau, 0)=d((\bar{x}, \tau), K \cap D)$, então vale

$$
|F(\bar{x}, \tau)| \leq C(K, m) d((\bar{x}, \tau), K \cap D)^{a m}, \quad \forall K \subset \mathbb{S}^{n-1} \times \mathbb{R}^{+}
$$

Portanto $F \in C_{\text {flat }}^{\infty}(U, D)$.

\subsection{Campos Singularmente Perturbados}

Nosso objetivo principal será estudar a extensão da Teoria Geométrica de Perturbação Singular(TGPS) às singularidades degeneradas. As Variedades Centrais (ver próximas seções) ao longo das variedades lentas constituem elementos relevantes desta teoria.

Definição 2.2.1 Uma familia $X_{\varepsilon, a}(x)=X(x, \varepsilon, a)$ de campos vetoriais sobre $\mathbb{R}^{n}$, com parâmetros $\varepsilon \in \mathbb{R}, a \in\left(\mathbb{R}^{n}, 0\right)$, será chamada de perturbação singular se o conjunto $Z=\operatorname{Sing}\left(X_{0, a}\right)$ de singularidades do campo $X_{0, a}$ é uma variedade de dimensão $\geq 1$. Esta variedade $Z$ é denominada variedade lenta.

Exemplo 2.2.2 Vejamos alguns exemplos

(1) A familia planar

$X_{\varepsilon, a}(x, y)=(x y+\varepsilon \cos (a))(\partial / \partial x)+(x y+\varepsilon \operatorname{sen}(a))(\partial / \partial y), \quad \varepsilon, a \in \mathbb{R}$ é uma perturbação singular com variedade lenta $Z=\{x y=0\}$.

(2) A equação de Van der Pol

$$
\left.X_{\varepsilon, a}(x, y)=(y-F(x))(\partial / \partial x)+\varepsilon(a-x)\right)(\partial / \partial y), \quad \varepsilon, a \in \mathbb{R}
$$

onde $F(x) \in \mathbb{R}[x]$, é uma perturbação singular com variedade lenta $Z=\{y=F(x)\}$.

(3) A perturbação singular

$$
X_{\varepsilon, a}(x, y)=\left(x^{2}-y^{2}+a \varepsilon\right)(\partial / \partial x)+\varepsilon(\partial / \partial y), \quad \varepsilon, a \in \mathbb{R}
$$

tem como variedade lenta $Z=\{(y-x)(y+x)=0\}$ 
(4) A perturbação singular

$$
X_{\varepsilon, a}(x, y)=\left(x\left(y-x^{2}\right)+a \varepsilon\right)(\partial / \partial x)+\varepsilon( \pm 1)(\partial / \partial y), \quad \varepsilon, a \in \mathbb{R}
$$

tem variedade lenta $Z=\{x=0\} \cup\left\{y=x^{2}\right\}$

Nosso estudo estará concentrado na análise local das singularidades degeneradas, nas perturbações singulares em $\mathbb{R}^{2}$ do tipo

$$
X_{\varepsilon, a}(x, y)=\varepsilon(\partial / \partial x)+F(x, y, \varepsilon, a)(\partial / \partial y), \quad(\varepsilon, a) \in \mathbb{R} \times\left(\mathbb{R}^{n}, 0\right)
$$

onde $(x, y) \in U_{x} \times U_{y} \subset(\mathbb{R}, 0) \times \mathbb{R}, F(x, y, \varepsilon, a)$ é uma função analítica em todas as variáveis tal que $F(x, y, 0,0)=y f(x, y)$ e $f$ analítica e não divisível por $y$.

\section{Observação 2.2.3 Notemos que}

- Esta familia satisfaz

$$
X_{0,0}(x, y)=Y(x, y)=y f(x, y) \frac{\partial}{\partial y}
$$

- Neste caso, a variedade lenta $Z=\operatorname{Sing}\left(X_{0,0}\right)$ é a curva $\Gamma=\{y f(x, y)=$ $0\}$ de singularidades não isoladas.

- A família $X_{\varepsilon, a}(x, y)$, pode ser olhada como sendo um campo vetorial $X(x, y, \varepsilon, a)$, sobre um aberto $U_{x, y, \varepsilon, a} \subset \mathbb{R}^{n+3}$

$$
X=\left\{\begin{array}{l}
\dot{y}=F(x, y, \varepsilon, a) \\
\dot{x}=\varepsilon \\
\dot{\varepsilon}=0 \\
\dot{a}=0
\end{array}\right.
$$

\subsubsection{Classificação das singularidades para $X_{\varepsilon, a}$}

\section{Singularidades Normalmente Hiperbólicas}

Seja $X$ um campo de vetores num aberto $U \subset \mathbb{R}^{n}$ e $\Gamma=\operatorname{Sing}(X)$ o seu conjunto de singularidades.

- Um ponto $q \in \Gamma$ será chamado de singularidade hiperbólica se

$$
\operatorname{Espec}(D X(q)) \cap i \mathbf{R}=\phi,
$$

ou seja todo autovalor $\lambda \in \operatorname{Espec}(D X(q))$ é tal que $\operatorname{Re}(\lambda) \neq 0$. 
- $q \in \Gamma$ será dita Normalmente Hiperbólica se $D X(q)$ tem pelo menos um autovalor com parte real diferente de zero.

Exemplo 2.2.4 Observemos alguns exemplos

(1) O conjunto de singularidades $\Gamma_{h}=\{y=0\} \cap\{x \mid f(x, 0) \neq 0\}$ do campo $Y(x, y)=y f(x, y)(\partial / \partial y)$ é normalmente hiperbólico pois a parte linear nos pontos $q=(x, 0) \in \Gamma_{h}$ é dada por

$$
\operatorname{DY}(\mathbf{q})=\left[\begin{array}{cc}
0 & 0 \\
0 & f(q)
\end{array}\right]
$$

Ou seja com a condição de que $F(x, y, 0,0)=y f(x, y)$, temos que $\Gamma=\{y=\varepsilon=a=0\}$ é uma curva de singularidades normalmente hiperbólicas para o campo $X_{\varepsilon, a}$ se $\frac{\partial}{\partial y} F(x, 0,0,0) \neq 0$.

(2) Para $\varepsilon=0$, o conjunto de singularidades

$$
\Gamma_{h}=\left\{y=\frac{x^{2}}{2}+\frac{x^{3}}{3}\right\} \backslash\{(-1,1 / 6),(0,0)\}
$$

para a familia Van der Pol

$$
X_{\varepsilon, a}(x, y)=\left(y-\left(\frac{x^{2}}{2}+\frac{x^{3}}{3}\right)\right)(\partial / \partial x)+\varepsilon(a-x)(\partial / \partial y), \quad \varepsilon, a \in \mathbb{R}
$$

é normalmente hiperbólico.

(3) Para $\varepsilon=0$, o conjunto de singularidades

$$
\Gamma_{h}=(\{x=0\} \cup\{y=0\}) \backslash\{(0,0)\}
$$

da familia

$X_{\varepsilon, a}(x, y)=(x y+\varepsilon \cos (a))(\partial / \partial x)+(x y+\varepsilon \operatorname{sen}(a))(\partial / \partial y), \quad \varepsilon, a \in \mathbb{R}$ é normalmente hiperbólico.

(4) Para $\varepsilon=0$, o conjunto de singularidades

$$
\Gamma_{h}=(\{y=x\} \cup\{y=-x\}) \backslash\{(0,0)\}
$$

para a famitia abaixo

$$
X_{\varepsilon, a}(x, y)=\left(x^{2}-y^{2}+\varepsilon a\right)(\partial / \partial x)+\varepsilon(\partial / \partial y), \quad \varepsilon, a \in \mathbb{R}
$$

em uma vizinhança da origem é normalmente hiperbólico. Tal familia é denominada familia Transcrítical. 
(5) Para $\varepsilon=0$, o conjunto de singularidades

$$
\Gamma_{h}=\left(\{x=0\} \cup\left\{y=x^{2}\right\}\right) \backslash\{(0,0)\}
$$

para a familia abaixo

$$
X_{\varepsilon, a}(x, y)=\left(x\left(y-x^{2}\right)+\varepsilon a\right)(\partial / \partial x)+\varepsilon( \pm 1)(\partial / \partial y), \quad \varepsilon, a \in \mathbb{R}
$$

em uma vizinhança da origem é normalmente hiperbólico. Tal familia é denominada familia Pitchfork.

\section{Singularidades Degeneradas}

Consideremos novamente a família $X_{\varepsilon, a}=\varepsilon \frac{\partial}{\partial x}+F(x, y, \varepsilon, a) \frac{\partial}{\partial y}$; definida em um domínio $U_{x} \times \mathbb{R}=U_{x} \times U_{y}$, onde $U_{x} \in(\mathbb{R}, 0)$ é uma vizinhança de zero, para cada $(\varepsilon, a) \in \mathbb{R} \times \mathbb{R}^{n}$; sendo $\Gamma=\{y=\varepsilon=a=0\} \approx U_{x}$ a curva lenta de $X_{0,0}$.

Uma singularidade $q \in \Gamma=\{y=\varepsilon=a=0\}=\operatorname{Sing}\left(X_{0,0}\right)$, é dita degenerada se não é normalmente hiperbólica. Denotemos por $\operatorname{Deg}\left(X_{0,0}\right) \subset$ $\Gamma$ o conjunto de singularidades degeneradas.

Seja $B_{x}:=f(x, 0)$. Então $B_{x}$ é o autovalor na direção vertical para o campo $X_{0,0}$, em $x \in \Gamma$. Logo $\operatorname{Deg}\left(X_{0,0}\right)=\left\{\left(x^{\prime}, 0\right) \in \Gamma \quad \mid B_{x^{\prime}}=0\right\}$

Com a ordenação induzida em $\Gamma \approx U_{x}$, temos transições de sinal para $B_{x}$ numa vizinhança $V\left(x^{\prime}\right)$ de $x^{\prime} \in \operatorname{Deg}\left(X_{0,0}\right)$ em $\Gamma$.

Vamos estabelecer a seguinte classificação: 
(a) Transição estável-estável $(s, s)$

$$
\forall x \in(\Gamma \backslash D e g) \cap V\left(x^{\prime}\right), \quad B_{x}<0
$$

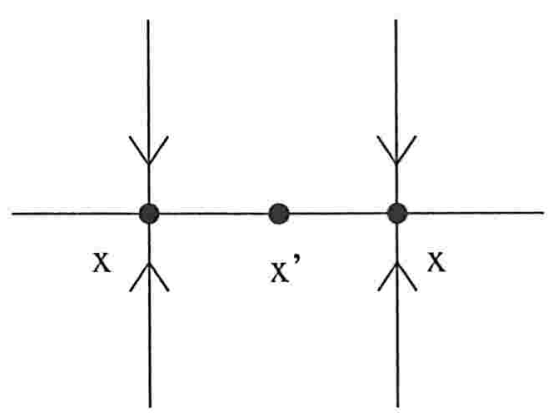

Figura 2.1: Transição estável-estável

(b) Transição instável-instável $(u, u)$

$$
\forall x \in(\Gamma \backslash D e g) \cap V\left(x^{\prime}\right), \quad B_{x}>0
$$

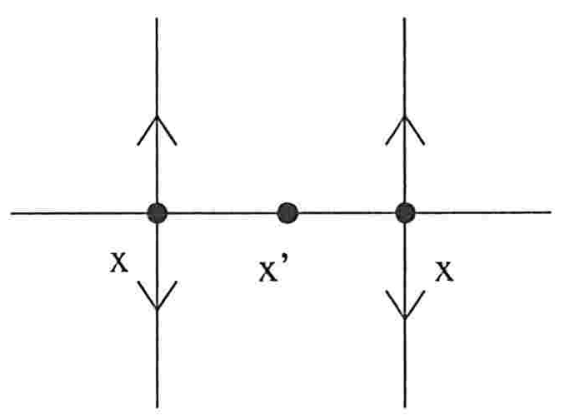

Figura 2.2: Transição instável-instável

(c) Transição instável-estável $(u, s)$

$$
\forall x \in(\Gamma \backslash D e g) \cap V\left(x^{\prime}\right), \quad x<x^{\prime}\left(\text { resp. } \quad x^{\prime}<x\right), \quad B_{x}>0\left(\text { resp. } \quad B_{x}<0\right)
$$




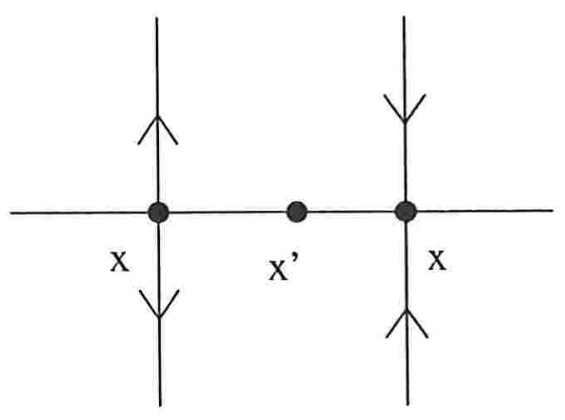

Figura 2.3: Transição instável-estável

(d) Transição estável-instável (s,u)

$$
\forall x \in(\Gamma \backslash D e g) \cap V\left(x^{\prime}\right), \quad x<x^{\prime}\left(\text { resp. } \quad x^{\prime}<x\right), \quad B_{x}<0\left(\text { resp. } \quad B_{x}>0\right)
$$

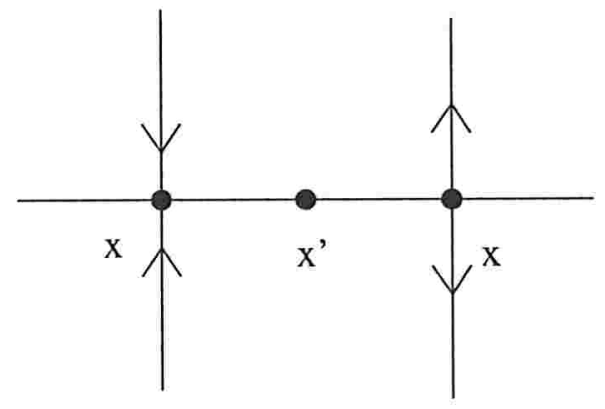

Figura 2.4: Transição estável-instável

Observação 2.2.5 Estas transições serão estendidas por continuidade, pela aplicação blowing-up $\Phi$ às singularidades normalmente hiperbólicas do campo $X \circ \Phi$ nas coordenadas blowing-up.

Exemplo 2.2.6 Para $\varepsilon=0$, a perturbação singular

$$
X_{\varepsilon}(x, y)=\varepsilon(\partial / \partial x)+(x y+\varepsilon)(\partial / \partial y), \quad \varepsilon \in \mathbb{R}
$$

apresenta a transição $(s, u)$, na singularidade degenerada $(0,0)$, pois $B_{x}=$ $x$, sendo neste caso $\Gamma \backslash$ Deg $=\{y=0\} \backslash\{(0,0)\}$ a curva normalmente 
hiperbólica.

Similarmente

$$
Y_{\varepsilon}(x, y)=\varepsilon(\partial / \partial x)+\left(x^{2} y+\varepsilon\right)(\partial / \partial y), \quad \varepsilon \in \mathbb{R}
$$

apresenta a transição $(u, u)$, em $(0,0)$ onde $B_{x}=x^{2}$.

Os campos $-X_{\varepsilon}(x, y),-Y_{\varepsilon}(x, y)$, apresentam respectivamente as transições $(u, s),(s, s)$ em $\quad(0,0)$.

\subsection{Hipóteses Gerais}

Sobre um aberto $U=U_{x} \times U_{y} \times U_{\varepsilon} \times U_{a} \subset\left(\mathbb{R}^{n+3}, 0\right)$, definamos o campo vetorial analítico singularmente perturbado

$$
X=X_{\varepsilon, a}(x, y)=\varepsilon(\partial / \partial x)+F(x, y, \varepsilon, a)(\partial / \partial y)
$$

com curva crítica $\Gamma=\{y=\varepsilon=a=0\} \approx U_{x} \subset(\mathbb{R}, 0)$.

\subsubsection{Hipóteses para $(X, \Gamma)$}

Consideremos a seguinte expansão em potências de $y$ para $F(x, y, \varepsilon, a)$

$$
F(x, y, \varepsilon, a)=F_{0}(x, \varepsilon, a)+F_{1}(x, \varepsilon, a) y+Q(x, y, \varepsilon, a) y^{2}
$$

tal que as funções $F_{0}$ e $F_{1}$ satisfazem às condições:

(i) $F_{0}(x, 0,0)=0, \quad \forall x \in \Gamma$

(ii) $F_{1}(0,0,0)=0$

(iii) $F_{1}(x, 0,0) \not \equiv 0, \quad \forall x \in \Gamma \backslash D e g$

isto implica que $D e g=\{0\}$ e $\Gamma \backslash D e g$ é normalmente hiperbólico.

Definamos agora o inteiro não negativo $\mu(X)(0)=p$ como sendo a multiplicidade da função $F_{1}(x, 0,0)$ em $x=0 ;$ o qual implicará que $\frac{1}{p !} \frac{\partial^{p} F_{1}}{\partial x^{p}}(0,0,0) \neq 0$.

\subsubsection{Hipóteses de Transversalidade para $(X, 0)$}

Consideremos as seguintes expansões em potências de $x$.

$$
\begin{aligned}
& F_{0}(x, \varepsilon, a)=\sum_{i=0}^{2 p-1} A_{i}(\varepsilon, a) x^{i}+A_{2 p}(\varepsilon, a) x^{2 p}+x^{2 p}\left(\sum_{i=2}^{\infty} A_{2 p+i-1}(\varepsilon, a) x^{i-1}\right), \\
& F_{1}(x, \varepsilon, a)=\sum_{j=0}^{p-1} B_{j}(\varepsilon, a) x^{j}+B_{p}(\varepsilon, a) x^{p}+x^{p}\left(\sum_{j=2}^{\infty} B_{p+i-1}(\varepsilon, a) x^{j-1}\right) .
\end{aligned}
$$


(1) Note que pelas hipóteses (i) e (ii) para $(X, \Gamma)$, temos

$$
\begin{aligned}
& A_{i}(0,0)=0, \quad \forall i \geq 0 \\
& B_{j}(0,0)=0, \quad \forall 0 \leq j \leq p-1, \quad B_{p}(0,0)=\frac{1}{p !} \frac{\partial^{p} F_{1}}{\partial x^{p}}(0,0,0) \neq 0 .
\end{aligned}
$$

(2) Diremos que a família $X=X_{\varepsilon, a}$ é transversal a $\Gamma$ se a função

$$
\begin{aligned}
\varphi: & \mathbb{R}^{n} \longrightarrow \mathbb{R}^{3 p} \\
& a \longmapsto\left(A_{0}(0, a), \ldots, A_{2 p-1}(0, a), B_{0}(0, a), \ldots, B_{p-1}(0, a)\right)
\end{aligned}
$$

for uma submersão em $a=0$. Neste caso, o teorema das funções implícitas garante que, a menos de permutação dos índices, a função $\Psi(\varepsilon, a)=\left(\varepsilon, A(\varepsilon, a), B(\varepsilon, a), \mathcal{A}_{r}\right)$ é um difeomorfismo local, onde $\mathcal{A}_{r}=$ $\left(a_{3 p+1}, \ldots, a_{n}\right)$ serão ditos de parâmetros não-essenciais e os novos parâmetros $\left(\varepsilon, a, b, \mathcal{A}_{r}\right)$ de parâmetros adaptados, onde

$$
\left\{\begin{array}{l}
a_{i}=A_{i}(\varepsilon, a), \quad 0 \leq i \leq 2 p-1 \\
b_{j}=B_{j}(\varepsilon, a), \quad 0 \leq j \leq p-1 \\
\mathcal{A}_{r} \in U_{\mathcal{A}_{r}} \subset\left(\mathbb{R}^{n}, 0\right)
\end{array}\right.
$$

\subsubsection{Blowing-up Principal}

Nas condições anteriores, o Blowing-up quase-homogêneo utilizado será

$$
\begin{aligned}
\Phi: \mathbb{S}^{3 p+2} \times \mathbb{R}^{n-3 p} \times \mathbb{R} & \longrightarrow \mathbb{R}^{2} \times \mathbb{R} \times \mathbb{R}^{3 p} \times \mathbb{R}^{n-3 p} \\
\left(\left(\bar{x}, \bar{y}, \bar{\varepsilon}, \bar{a}_{i}, \bar{b}_{j}\right), \mathcal{A}_{r}, \tau\right) & \longrightarrow\left((x, y), \varepsilon,\left(a_{i}, b_{j}\right), \mathcal{A}_{r}\right)
\end{aligned}
$$

descrito pela correspondência

$$
\Phi=\left\{\begin{array}{l}
x=\tau \bar{x} \\
y=\tau^{p} \bar{y} \\
\varepsilon=\tau^{p+1} \bar{\varepsilon} \\
a_{i}=\tau^{2 p-i} \bar{a}_{i}, \quad i=0, \ldots, 2 p-1 \\
b_{j}=\tau^{p-j} \bar{b}_{j}, \quad j=0, \ldots, p-1 \\
\mathcal{A}_{r}=\mathcal{A}_{r}
\end{array}\right.
$$

onde $p=\mu(X)(0)$. Tal transformação será chamada de Blowing-up principal. A família $\bar{X}$ será dada por

$$
\bar{X}=\left(\frac{1}{\tau^{p}}\right) X \circ \Phi
$$

No capítulo 4 estudaremos a expressão de $\bar{X}$ em detalhes. 


\section{Capítulo 3}

\section{Variedades Centrais sobre $\Gamma \backslash \operatorname{Deg}(X)$}

\subsection{Linearização}

Aqui fazemos a construção de variedades invariantes pelo fluxo de um campo vetorial singularmente perturbado $X$, da forma $X=\varepsilon \frac{\partial}{\partial x}+F(x, y, \varepsilon, \mathcal{A}) \frac{\partial}{\partial y}$ definido num aberto de $\mathbb{R}^{2}$. Tal variedade será definida por uma função condição inicial.

Consideremos os seguintes domínios

$$
\left\{\begin{aligned}
U_{x} & \in(\mathbb{R}, 0), \text { um conjunto aberto e conexo, } \\
U_{y} & =\mathbb{R} \\
U_{\varepsilon} & \in\left(\mathbb{R}^{+}, 0\right) \\
U_{\mathcal{A}} & \in\left(\mathbb{R}^{n}, 0\right) .
\end{aligned}\right.
$$

E suponhamos que $X$ está definido sobre o aberto

$$
U=U_{x} \times U_{y} \times U_{\varepsilon} \times U_{\mathcal{A}}
$$

satisfazendo as seguintes condições

(i) $F \in C^{w}(U)$,

(ii) $F(x, 0,0,0) \equiv 0$,

(iii) $B_{x}=\frac{\partial F}{\partial y}(x, 0,0,0)$, é tal que $\left|B_{x}\right|>\delta>0$, para algum $\delta>0$.

Daqui notemos que 
1. (i),(ii) implicam que a subvariedade

$$
\Gamma=\{y=\varepsilon=\mathcal{A}=0\} \approx U_{x}
$$

é uma curva de singularidades de $X$ em $\mathrm{U}$.

2. (iii) implica que $\Gamma$ é uma subvariedade invariante normalmente hiperbólica; para ver isto é suficiente olhar a parte linear do campo no ponto $(x, 0,0,0)$ na base $\left\{\frac{\partial}{\partial x}, \frac{\partial}{\partial y}\right\}$

$$
\operatorname{DX}(x, 0,0,0)=\left[\begin{array}{cc}
0 & 0 \\
\frac{\partial F}{\partial x}(x, 0,0,0) & \frac{\partial F}{\partial y}(x, 0,0,0)
\end{array}\right]=\left[\begin{array}{cc}
0 & 0 \\
0 & B_{x}
\end{array}\right] .
$$

3. Pela conexidade de $\Gamma \approx U_{x}, B_{x}$ não muda de sinal, para todo $x \in \Gamma$

4. O campo $X=\varepsilon(\partial / \partial x)+F(x, y, \varepsilon, \mathcal{A})(\partial / \partial y)$ tem associado uma equação diferencial de primeira ordem.

$$
\left\{\begin{array}{l}
\dot{x}=\varepsilon \\
\dot{y}=F(x, y, \varepsilon, \mathcal{A})
\end{array}\right.
$$

para cada $(x, y) \in U_{x} \times U_{y}$, dependendo analíticamente dos parâmetros $(\varepsilon, \mathcal{A}) \in U_{\varepsilon} \times U_{\mathcal{A}}$.

Equivalentemente podemos escrever a equação diferencial

$$
\left\{\begin{array}{l}
\dot{x}=\varepsilon \\
\dot{y}=F(x, y, \varepsilon, \mathcal{A}) \\
\dot{\varepsilon}=0 \\
\dot{\mathcal{A}}=0
\end{array}\right.
$$

para cada $(x, y, \varepsilon, \mathcal{A}) \in U_{x} \times U_{y} \times U_{\varepsilon} \times U_{\mathcal{A}}$. A parte linear de $X$ no ponto $(x, 0,0,0,) \in \Gamma \approx U_{x}$ é dada por

$$
L=\operatorname{DX}(x, 0,0,0)=\left[\begin{array}{cccc}
0 & 0 & 1 & 0 \\
0 & B_{x} & F_{\varepsilon} & F_{\mathcal{A}} \\
0 & 0 & 0 & 0 \\
0 & 0 & 0 & 0
\end{array}\right]
$$

Como $\left|B_{x}\right|>\delta>0$, o espectro de $L$ no espaço $\mathbb{R}^{n+3}=\mathbb{R} \times \mathbb{R} \times \mathbb{R}^{n+1}$, pode ser decomposto como

$$
\sigma(L)=\sigma_{s}(L) \cup \sigma_{c}(L) \cup \sigma_{u}(L)
$$


onde

$$
\begin{aligned}
& \sigma_{s}(L)=\{\lambda=\alpha+i \beta: \alpha<0\}, \\
& \sigma_{c}(L)=\{\lambda=\alpha+i \beta: \alpha=0\}, \\
& \sigma_{u}(L)=\{\lambda=\alpha+i \beta: \alpha>0\} .
\end{aligned}
$$

Notemos que o subespaço $\mathbb{R}_{x, \varepsilon, \mathcal{A}}^{n+2}$ é gerado pelos autovetores correspondentes aos autovalores $\lambda \in \sigma_{c}(L)$, e $\mathbb{R}_{y}=\mathbb{R}$ é gerado pelos autovetores correspondentes aos autovalores $B_{x} \in \sigma_{s}(L) \cup \sigma_{u}(L)$. Assim

- se $B_{x}>0, \quad$ então $\sigma_{s}(L)=\phi, \quad$ e $\mathbb{R}_{y} \quad$ é gerado por autovetores correspondentes a $B_{x} \in \sigma_{u}(L)$.

- se $B_{x}<0$, então $\sigma_{u}(L)=\phi, \quad$ e $\mathbb{R}_{y} \quad$ é gerado por autovetores correspondentes a $B_{x} \in \sigma_{s}(L)$.

Agora colocando $U_{x, \varepsilon, \mathcal{A}}=U_{x} \times U_{\varepsilon} \times U_{\mathcal{A}}=U \cap\{y=0\}$, o teorema da variedade central local implica no seguinte resultado:

Proposição 3.1.1 Seja $x \in \Gamma$ um ponto arbitrário. Então para cada $k \in \mathbb{N}$ existe uma vizinhança $V_{x, \varepsilon, \mathcal{A}}$ de $x$ em $U_{x, \varepsilon, \mathcal{A}}$ e uma função $C^{k}\left(V_{x, \varepsilon, \mathcal{A}}, \mathbb{R}\right)$

$$
\begin{aligned}
w: & V_{x, \varepsilon, \mathcal{A}} \longrightarrow U_{y} \\
& (x, \varepsilon, \mathcal{A}) \longmapsto y=w(x, \varepsilon, \mathcal{A})
\end{aligned}
$$

tal que

- $w$ é definida em $V_{x, \varepsilon, \mathcal{A}}$,

- $w(x, 0,0) \equiv 0$,

- $W=\operatorname{graf}\{y=w(x, \varepsilon, \mathcal{A})\}$, é uma variedade invariante por $X$.

DEMONSTRAÇÃo

Ver $[\mathrm{Ak}],[\mathrm{JS}]$.

$W$ será dita uma variedade central local em $x$.

Observação 3.1.2 Consideremos $X(x, y)=\varepsilon \frac{\partial}{\partial x}-y \frac{\partial}{\partial y}$. As variedades invariantes por $X$, neste caso

$$
W=\operatorname{graf}\{y=w(x, \Xi)\}, \forall x \in \mathbb{R}, \varepsilon>0
$$

são dadas por $W=\operatorname{graf}\left\{y=i(\varepsilon) e^{\frac{-x+x_{0}}{\varepsilon}}\right\}, \operatorname{com} i(\varepsilon) \in C^{\infty}, i(0)=0$ e $x_{0}<x$ arbitrário. 


\subsection{Variedades Centrais Formais}

Proposição 3.2.1 Seja $x \in \Gamma \approx U_{x}, W=\operatorname{graf}\{y=w(x, \varepsilon, \mathcal{A})\}$ uma variedade central local em $x$. Então existem vizinhanças abertas $U_{0}$ de $x e$ $U_{\mathcal{A}} \in\left(\mathbb{R}^{n}, 0\right)$, e funções analíticas $w_{i}(x, \mathcal{A}) \in C^{w}\left(U_{x} \times U_{\mathcal{A}}\right), i \geq 0$, definindo uma única série formal

$$
\widehat{W}(x, \varepsilon, \mathcal{A})=\sum_{i=0}^{\infty} w_{i}(x, \mathcal{A}) \varepsilon^{i}, \quad \varepsilon \in U_{\varepsilon} \in\left(\mathbb{R}^{+}, 0\right)
$$

tal que $w \in C_{\text {flat }}^{k}\left(U_{0} \times U_{\mathcal{A}},\{\varepsilon=0\}, \widehat{W}\right)$.

DEMONSTRAÇÃO

Observe que esta série pode ser escrita como

$$
\widehat{W}(x, \varepsilon, \mathcal{A})=w_{0}(x, \mathcal{A})+\widehat{M}(x, \varepsilon, \mathcal{A})
$$

onde

$$
\widehat{M}(x, \varepsilon, \mathcal{A})=\sum_{i=1}^{\infty} w_{i}(x, \mathcal{A}) \varepsilon^{i} .
$$

Assim, usando o teorema da função implícita e um argumento indutivo, a demonstração será feita basicamente em dois passos:

1. (Primeiro Passo)

Note que para o campo

$$
X(x, y, \varepsilon, \mathcal{A})=\varepsilon(\partial / \partial x)+F(x, y, \varepsilon, \mathcal{A})(\partial / \partial y), \quad \varepsilon \in \mathbb{R}, \mathcal{A} \in\left(\mathbb{R}^{n}, 0\right)
$$

o conjunto de singularidades

$$
\begin{aligned}
Z(X) & =\{(x, y, \varepsilon, \mathcal{A}): X(x, y, \varepsilon, \mathcal{A})=0\} \\
& =\{(x, y, \varepsilon, \mathcal{A}): \varepsilon=0, \quad F(x, y, 0, \mathcal{A})=0\},
\end{aligned}
$$

é uma subvariedade em $U=U_{x} \times U_{y} \times U_{\varepsilon} \times U_{\mathcal{A}}$ de codimensão $2=$ $\operatorname{dim}(\{(y, \varepsilon)\})$, perto de $\Gamma=\{y=\varepsilon=\mathcal{A}=0\}$.

Como o campo $X$ satisfaz à hipótese $\left|\frac{\partial F}{\partial y}(x, 0,0,0)\right|>\delta>0$. fixado $(x, \mathcal{A})=(x, 0)\left(Z(X) \subset\{(x, \mathcal{A})\}=U_{x} \times \mathbb{R}^{n}\right)$ existe uma vizinhança aberta conexa $U_{\mathcal{A}}^{0} \subset U_{\mathcal{A}}$ de $\mathcal{A}=0$, tal que

$$
\frac{\partial F}{\partial y}(x, 0,0, \mathcal{A}) \neq 0, \quad x \in U_{x}, \quad \mathcal{A} \in U_{\mathcal{A}}^{0},
$$


daqui pelo teorema da função implícita, existe uma única função analítica $y=h(x, 0, \mathcal{A})$, definida sobre $U_{x} \times\{0\} \times U_{\mathcal{A}}^{0}$, tal que $F(x, h, \varepsilon, \mathcal{A})=0$. Definindo $w_{0}(x, \mathcal{A}):=h(x, 0, \mathcal{A})$, fica mostrada a existência da função $w_{0}$ tal que

$$
Z(X)=\left\{(x, y, \varepsilon, \mathcal{A}): \varepsilon=0, \quad y=w_{0}(x, \mathcal{A})\right\} .
$$

Note que, fazendo a mudança analítica de coordenadas

$$
\left\{\begin{array}{l}
y=y^{\prime}-w_{0}(x, \mathcal{A}) \\
x=x^{\prime}
\end{array}\right.
$$

temos $F(x, y, \varepsilon, \mathcal{A})=F\left(x, y^{\prime}-w_{0}(x, \mathcal{A}), \varepsilon, \mathcal{A}\right)$, e na base $\left\{\frac{\partial}{\partial x}, \frac{\partial}{\partial y^{\prime}}\right\}, \mathrm{o}$ campo $X$ tem a nova expressão

$$
X=\varepsilon \frac{\partial}{\partial x}+F^{\prime}(x, y, \varepsilon, \mathcal{A}) \frac{\partial}{\partial y^{\prime}}
$$

onde $F^{\prime}(x, y, \varepsilon, \mathcal{A})=F\left(x, y^{\prime}-w_{0}(x, \mathcal{A}), \varepsilon, \mathcal{A}\right)+(\partial / \partial x) w_{0}(x, \mathcal{A}) \varepsilon$.

Daqui claramente obtemos:

$$
\begin{gathered}
F^{\prime}(x, 0,0, \mathcal{A})=F(x, 0,0, \mathcal{A})=0 \quad e \\
\left|\frac{\partial}{\partial y^{\prime}} F^{\prime}(x, 0,0, \mathcal{A})\right|=\left|\frac{\partial}{\partial y} F(x, 0,0, \mathcal{A})\right|>\delta .
\end{gathered}
$$

Observamos que as hipóteses para o campo $X=(\varepsilon, F)$ são preservadas pela mudança analítica no espaço de fase $\left(x^{\prime}, y^{\prime}\right)$ dado acima.

2. (Segundo Passo: Existência de $w_{i}, i \geq 1$ )

Vamos mostrar a existência das funções $w_{i}(x, \mathcal{A}), i \geq 1$, recursivamente. Para tal vamos escrever a série

$$
\widehat{M}(x, \varepsilon, \mathcal{A})=\sum_{i=1}^{\infty} w_{i}(x, \mathcal{A}) \varepsilon^{i}
$$

onde $w_{i} \in \mathbb{C}^{w}\left(U_{x} \times U_{\mathcal{A}}^{0}\right)$.

Vamos ilustrar o procedimento calculando $w_{1}(x, \mathcal{A})$.

Consideremos a série(mudança analítica)

$$
\widehat{K}(x, y, \Xi, \mathcal{A})=y-\widehat{M}(x, \Xi, \mathcal{A}) .
$$


Olhando o campo $X$ como operador derivação sobre o conjunto das séries formais, note por um lado que

$$
\left.X(\widehat{K})(x, y, \varepsilon, \mathcal{A})\right|_{y=\widehat{M}(x, \varepsilon, \mathcal{A})}=\varepsilon \frac{\partial}{\partial x}[y-\widehat{M}]+F \frac{\partial}{\partial y}[y-\widehat{M}]=0
$$

e por outro lado temos

$$
\begin{aligned}
\varepsilon \frac{\partial}{\partial x}[y-\widehat{M}] & =-\varepsilon \frac{\partial}{\partial x} \widehat{M}, \\
F \frac{\partial}{\partial y}[y-\widehat{M}] & =F .
\end{aligned}
$$

Portanto obtemos a relação formal

$$
\varepsilon \frac{\partial}{\partial x} \widehat{M}(x, \varepsilon, \mathcal{A})=F(x, \widehat{M}, \varepsilon, \mathcal{A}) .
$$

Daqui sendo $F$ analítica em todos seus termos consideremos a seguinte expansão em $y$.

$$
F(x, y, \varepsilon, \mathcal{A})=\sum_{i=0}^{\infty} f_{i}(x, \varepsilon, \mathcal{A}) y^{i}
$$

Como $y=\widehat{M}$, obtemos

$$
\varepsilon \frac{\partial}{\partial x} \widehat{M}(x, \varepsilon, \mathcal{A})=f_{0}(x, \varepsilon, \mathcal{A})+\widehat{M}(x, \varepsilon, \mathcal{A})\left[f_{1}(x, \varepsilon, \mathcal{A})+O(\varepsilon)\right]
$$

Com a finalidade de relacionar coeficientes de ambos lados desta igualdade, tomemos as expansões

$$
\begin{aligned}
& f_{0}(x, \varepsilon, \mathcal{A})=\sum_{j=0}^{\infty} f_{0, j}(x, \mathcal{A}) \varepsilon^{j} \quad e \\
& f_{1}(x, \varepsilon, \mathcal{A})=\sum_{j=0}^{\infty} f_{1, j}(x, \mathcal{A}) \varepsilon^{j} .
\end{aligned}
$$

Lembremos que pela primeira parte vale

$$
F(x, 0,0, \mathcal{A})=0, \quad\left|\frac{\partial}{\partial y} F(x, 0,0, \mathcal{A})\right|>\delta>0, \quad \mathcal{A} \in U_{\mathcal{A}}^{0} .
$$

De onde obtemos que

$$
f_{0}(x, 0, \mathcal{A})=0, \quad(x, \mathcal{A}) \in U_{x} \times U_{\mathcal{A}}^{0}
$$


e conseqüentemente

$$
f_{0,0}(x, \mathcal{A})=0, \quad \forall(x, \mathcal{A}) \in U_{x} \times U_{\mathcal{A}}^{0} .
$$

Notemos também que vale

$$
\frac{\partial}{\partial y} F(x, y, \varepsilon, \mathcal{A})=f_{1}(x, \varepsilon, \mathcal{A})+\sum_{i=2}^{\infty} i f_{i}(x, \varepsilon, \mathcal{A}) y^{i-1}
$$

de onde obtemos

$$
\frac{\partial}{\partial y} F(x, 0,0, \mathcal{A})=f_{1}(x, 0, \mathcal{A}) .
$$

Conseqüentemente da expansão de $f_{1}$ vale

$$
f_{1,0}(x, \mathcal{A})=f_{1}(x, 0, \mathcal{A})=\frac{\partial}{\partial y} F(x, 0,0, \mathcal{A}) \neq 0 .
$$

Das expressões acima temos

$$
\begin{aligned}
\varepsilon \frac{\partial}{\partial x} \widehat{M} & =\varepsilon \sum_{j=1}^{\infty} \frac{\partial}{\partial x} w_{j}(x, \mathcal{A}) \varepsilon^{j}, \\
f_{0}(x, \varepsilon, \mathcal{A}) & =\varepsilon f_{0,1}(x, \mathcal{A})+\varepsilon \sum_{j=2}^{\infty} f_{0, j}(x, \mathcal{A}) \varepsilon^{j-1}, \\
\widehat{M}\left(f_{1}+O(\varepsilon)\right) & =\varepsilon w_{1} f_{1,0}+O\left(\varepsilon^{2}\right) .
\end{aligned}
$$

Logo, a equação formal

$$
\varepsilon \frac{\partial}{\partial x} \widehat{M}=\widehat{M}\left(f_{1}+O(\varepsilon)\right)
$$

pode ser escrita como

$$
\varepsilon \sum_{j=1}^{\infty} \frac{\partial}{\partial x} w_{j} \varepsilon^{j}=\varepsilon f_{0,1}+\varepsilon w_{1} f_{1,0}+O\left(\varepsilon^{2}\right)
$$

ou também como

$$
O\left(\varepsilon^{2}\right)=\varepsilon f_{0,1}+\varepsilon w_{1} f_{1,0}+O\left(\varepsilon^{2}\right) .
$$

Agora fazendo $\varepsilon=0$, após uma divisão por $\varepsilon$ nesta última igualdade, obtemos

$$
0=f_{0,1}+w_{1} f_{1,0} .
$$


Logo podemos definir $w_{1}$ como sendo

$$
w_{1}(x, \mathcal{A})=\frac{-f_{0,1}(x, \mathcal{A})}{f_{1,0}(x, \mathcal{A})}, \quad \forall(x, \mathcal{A}) \in U_{x} \times U_{\mathcal{A}}^{0} .
$$

Notemos que $w_{2}$ pode-se obter, dependendo de $w_{1}$ e dos coeficientes $f_{i j},(\mathrm{i}, \mathrm{j}=0,1)$.

De maneira similar é possível determinar recursivamente as funções $w_{i}$ dependendo de $f_{l, j}$ e $w_{j},(l=0,1 ; j<i)$ (o fato importante neste procedimento é o uso sucessivo do teorema da função implícita nas mudanças analíticas do plano fase). Isto encerra a demonstração.

Observação 3.2.2 Em geral, a série formal $\widehat{W}$ é divergente.

\subsection{Variedade Central Dinâmica}

Observação 3.3.1 Para algum conjunto fechado $M \subset U_{x} \times U_{\mathcal{A}}$, é possivel mostrar que sobre o conjunto fechado $\{\varepsilon=0\} \times M$ vale

$$
\frac{D^{i} \widehat{W}}{i !}(x, 0, \mathcal{A})=w_{i}(x, \mathcal{A}), \quad \forall(x, \mathcal{A}) \in M, \quad i \geq 0
$$

onde $D^{i}$ é o operador derivação de ordem $i$.

Logo a serie $\widehat{W}(x, \varepsilon, \mathcal{A})=\sum_{i=0}^{\infty} w_{i}(x, \mathcal{A}) \varepsilon^{i}$ identifica-se com um único elemento de $C^{\infty}(\{\varepsilon=0\} \times M)$.

Assim, para cada ponto $x^{\prime} \in \Gamma \approx U_{x}$, podemos restringir a variedade central formal $\widehat{W}(x, \varepsilon, \mathcal{A})$ a $\left\{x=x^{\prime}\right\}$, dada pela função

$$
\widehat{W}_{x^{\prime}}:=\widehat{W}\left(x^{\prime}, \varepsilon, \mathcal{A}\right)=\sum_{i=0}^{\infty} w_{i}\left(x^{\prime}, \mathcal{A}\right) \varepsilon^{i}, \quad \forall(\varepsilon, \mathcal{A}) \in U_{\varepsilon} \times U_{\mathcal{A}} .
$$

Definição 3.3.2 - Dado um conjunto aberto $V \subset\left(U_{\varepsilon} \cap\{\varepsilon>0\}\right) \times U_{\mathcal{A}}$. A função $i: V \longrightarrow U_{y} \in C^{\infty}$ é chamada função condição inicial para o campo $X$ em $x^{\prime} \in \Gamma$ se

$$
i \in C_{\text {flat }}^{\infty}\left(V,\{\varepsilon=0\}, \widehat{W}_{x^{\prime}}\right)
$$

ou seja para algum subconjunto compacto $K \subset V$

$$
\begin{aligned}
& \left|\left(i-\widehat{W}_{x^{\prime}}\right)(\varepsilon, \mathcal{A})\right| \leq C|(\varepsilon, \mathcal{A})-(0, \mathcal{A})|^{\alpha}=C|\varepsilon|^{\alpha}, \quad \forall(\varepsilon, \mathcal{A}) \in K \\
& \text { onde } C=C(K) \text { e } \alpha \text { são constantes positivas, e } K-\{\varepsilon=0\} \subset V
\end{aligned}
$$


- Para $x_{0}, x_{1} \in \Gamma, x_{0}<x_{1}$, definimos $\Gamma_{x_{0}, x_{1}}=\left[x_{0}, x_{1}\right]$

Proposição 3.3.3 Seja $x_{0} \in \Gamma, i \in C_{\text {flat }}^{\infty}\left(V,\{\varepsilon=0\}, \widehat{W}_{x_{0}}\right)$ uma função condição inicial arbitrária para o campo $X$ em $x_{0}$ e seja $B_{x_{0}}<0$, (ou seja $D X\left(x_{0}\right)$ é hiperbólico estável). Então para todo $x_{1}>x_{0}$ suficientemente próximo, existe uma vizinhança $N \subset U_{\varepsilon, \mathcal{A}}$ da origem $(\varepsilon, \mathcal{A})=(0,0)$ tal que considerando a restrição de $i$ ao conjunto

$$
\mathcal{O}:=V \cap N
$$

existe uma única função $w(x, \varepsilon, \mathcal{A}) \in C^{\infty}$ definida sobre $\Gamma_{x_{0}, x_{1}} \times \mathcal{O}$ tal que

(i) $w\left(x_{0}, \varepsilon, \mathcal{A}\right)=i(\varepsilon, \mathcal{A})$;

(ii) $W=\operatorname{graf}(y=w(x, \varepsilon, \mathcal{A}))$, é uma variedade invariante por $X$;

(iii) $w \in C_{\text {flat }}^{\infty}\left(\Gamma_{x_{0}, x_{1}} \times \mathcal{O},\{\varepsilon=0\}, \widehat{W}\right)$.

DEMONSTRAÇÃO

É suficiente considerar alguma $C^{k}$ variedade central

$$
W^{\prime}=\operatorname{graf}\left\{y=h^{\prime}(x, \varepsilon, \mathcal{A})\right\}
$$

em algum ponto $x \in \Gamma_{x_{0}, x_{1}} ;$ e mostrar que

$$
w^{\prime}=w-h^{\prime} \in C_{\text {flat }}^{\infty}\left(U^{\prime},\{\varepsilon=0\}\right), \quad U^{\prime} \subset \Gamma_{x_{0}, x_{1}} \times \mathcal{O} .
$$

Com efeito, pela teoria de formas normais para campos singularmente perturbados (ver Apêndice de [DP]), temos que a família de campos de vetores

$$
X_{\varepsilon, \mathcal{A}}=\varepsilon(\partial / \partial x)+F(x, y, \varepsilon, \mathcal{A})(\partial / \partial y), \quad \varepsilon \in \mathbb{R}, \mathcal{A} \in\left(\mathbb{R}^{n}, 0\right)
$$

é $C^{k}$-equivalente ao campo

$$
Y\left(x^{\prime}, y^{\prime}, \varepsilon^{\prime}\right)=\varepsilon^{\prime}\left(\partial / \partial x^{\prime}\right)+s y^{\prime}\left(\partial / \partial y^{\prime}\right), \quad \varepsilon \in \mathbb{R}, s \in\{-1,1\}
$$

por uma mudança local de coordenadas de classe $C^{k}$

$$
(x, y, \varepsilon, \mathcal{A}) \longrightarrow\left(x^{\prime}, y^{\prime}, \varepsilon^{\prime}, \mathcal{A}^{\prime}\right)=\left(x^{\prime}, y-h^{\prime}, \varepsilon^{\prime}, \mathcal{A}^{\prime}\right) .
$$

Nestas coordenadas temos

$$
W^{\prime}=\left\{y^{\prime}=0\right\}, \quad y_{\varepsilon^{\prime}, \mathcal{A}^{\prime}}^{\prime}\left(x_{0}^{\prime}\right)=i^{\prime}\left(\varepsilon^{\prime}, \mathcal{A}^{\prime}\right), \quad i^{\prime} \in C_{\text {flat }}^{\infty}\left(U^{\prime},\left\{\varepsilon^{\prime}=0\right\}\right) .
$$


Para $x_{0}^{\prime}=0$, as trajectórias de $Y$ passando pelo ponto inicial $\left(x_{0}^{\prime}, y_{0}^{\prime}\right)=$ $\left(0, i^{\prime}\left(\varepsilon^{\prime}, \mathcal{A}^{\prime}\right)\right)$ são dadas por

$$
\left\{\begin{array}{l}
x^{\prime}(t)=\varepsilon^{\prime} t \\
y^{\prime}(t)=\exp (-t) i^{\prime}\left(\varepsilon^{\prime}, \mathcal{A}^{\prime}\right)
\end{array}\right.
$$

Assim $w^{\prime}=w-h^{\prime}$ pode ser definida por

$$
w^{\prime}\left(x^{\prime}, \varepsilon^{\prime}, \mathcal{A}^{\prime}\right)=\left\{\begin{array}{lll}
i^{\prime}\left(\varepsilon^{\prime}, \mathcal{A}^{\prime}\right) & \text {, se } & x^{\prime}=0 \\
\exp \left(-\frac{x^{\prime}}{\varepsilon^{\prime}}\right) i^{\prime}\left(\varepsilon^{\prime}, \mathcal{A}^{\prime}\right) & \text {, se } & x^{\prime}>0
\end{array}\right.
$$

Sabemos que a função $g\left(x^{\prime}, \varepsilon^{\prime}\right)=\exp \left(-\frac{x^{\prime}}{\varepsilon^{\prime}}\right)$, é uniformemente limitada na região $\left\{\left(x^{\prime}, \varepsilon^{\prime}\right) \mid x^{\prime} \leq 0, \quad \varepsilon^{\prime} \leq 0\right\}$; similarmente suas derivadas parciais

$$
\frac{\partial^{s_{1}+s_{2}} g}{\partial^{s_{1}} x^{\prime} \partial^{s_{2}} \varepsilon^{\prime}}
$$

são uniformemente limitadas; por exemplo no caso $s_{1}=2, s_{2}=3$, obtemos

$$
\begin{aligned}
\frac{\partial^{s_{1}+s_{2}} g}{\partial^{s_{1}} x^{\prime} \partial^{s_{2}} \varepsilon^{\prime}} & \approx g\left(x^{\prime}, \varepsilon^{\prime}\right)\left(\frac{x^{\prime 3}}{\varepsilon^{\prime 8}}-12 \frac{x^{\prime 2}}{\varepsilon^{\prime 7}}+36 \frac{x^{\prime}}{\varepsilon^{\prime 6}}-24 \frac{1}{\varepsilon^{\prime 5}}\right) \\
& =\exp \left(-\frac{x^{\prime}}{\varepsilon^{\prime}}\right) P\left(\frac{x^{\prime}}{\varepsilon^{\prime}}\right) \frac{1}{\varepsilon^{\prime 5}}
\end{aligned}
$$

onde $\operatorname{deg}(P)=s_{2}$, e como é sabido do cálculo que

$$
\left\{\begin{array}{lll}
\exp \left(-\frac{x^{\prime}}{\varepsilon^{\prime}}\right) P\left(\frac{x^{\prime}}{\varepsilon^{\prime}}\right) \longrightarrow 0 & , \quad x^{\prime} \longrightarrow+\infty ; \\
\exp \left(-\frac{x^{\prime}}{\varepsilon^{\prime}}\right) P\left(\frac{x^{\prime}}{\varepsilon^{\prime}}\right) \longrightarrow 0 & , \quad \varepsilon^{\prime} \longrightarrow 0 ; \\
\exp \left(-\frac{x^{\prime}}{\varepsilon^{\prime}}\right) P\left(\frac{x^{\prime}}{\varepsilon^{\prime}}\right) \longrightarrow \text { Cte } & , \quad \varepsilon^{\prime} \longrightarrow+\infty
\end{array}\right.
$$

para todo polinomio $P\left(\frac{x^{\prime}}{\varepsilon^{\prime}}\right)$, com $\operatorname{deg}(P)=n \geq \max \left\{s_{1}, s_{2}\right\}$; logo poderíamos obter constantes positivas, $C=C\left(s_{1}, s_{2}\right), \alpha=\alpha\left(s_{1}, s_{2}\right), \beta=$ $\beta\left(s_{1}, s_{2}\right)$, tal que

$$
\left|\frac{\partial^{s_{1}+s_{2}} g}{\partial^{s_{1}} x^{\prime} \partial^{s_{2}} \varepsilon^{\prime}}\right| \leq \frac{C}{x^{\prime \alpha} \varepsilon^{\prime \beta}},
$$

Assim, como $i^{\prime}\left(\varepsilon^{\prime}, \mathcal{A}^{\prime}\right) \in C_{\text {flat }}^{\infty}\left(U^{\prime},\left\{\varepsilon^{\prime}=0\right\}\right)$, temos que

$$
i^{\prime}\left(\varepsilon^{\prime}, \mathcal{A}^{\prime}\right) g\left(x^{\prime}, \varepsilon^{\prime}\right) \in C_{\text {flat }}^{\infty}\left(U^{\prime},\left\{\varepsilon^{\prime}=0\right\}\right),
$$

de onde

$$
w-h^{\prime}=w^{\prime} \in C_{\text {flat }}^{\infty}\left(U^{\prime},\left\{\varepsilon^{\prime}=0\right\}\right) .
$$

Isto conclui a prova (veja Figura 3.1). 


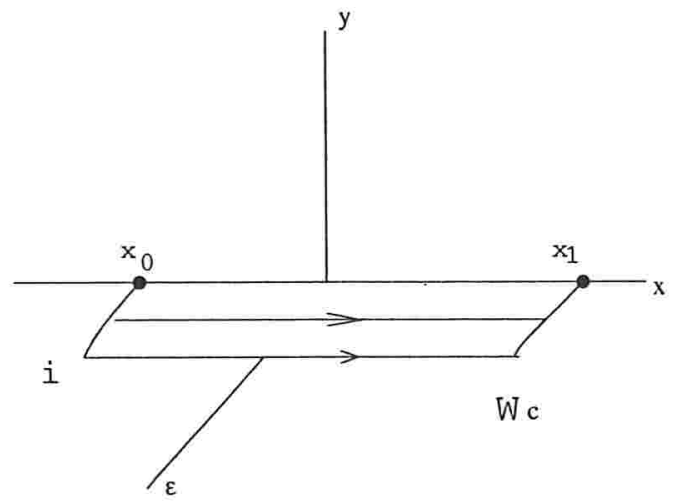

Figura 3.1: Variedade Central Dinâmica Normalmente Hiperbólica.

Observação 3.3.4 1. Para cada $x_{1}<x_{0}$, com $B_{x_{0}}>0$, usando o fluxo com tempo negativo mostraríamos o resultado com as mesmas conclusões.

2. Colocando $\tilde{\Gamma}=\Gamma_{x_{0}, x_{1}}$, o conjunto $\mathcal{O}_{\tilde{\Gamma}} \subset U_{\varepsilon, \mathcal{A}}$ é chamado de região Canard e $W_{\tilde{\Gamma}}$ é chamada de variedade central dinâmica sobre $\tilde{\Gamma}$.

3. Para singularidades semi-hiperbólicas sempre existem $\mathcal{O}_{\bar{\Gamma}}$ e $W_{\tilde{\Gamma}}$. 
44 CAPÍTULO 3. VARIEDADES CENTRAIS SOBRE $\Gamma \backslash D E G(X)$ 


\section{Capítulo 4}

\section{Geometria no espaço fase de $\bar{X}$}

Daremos explicitamente a estrutura analítica para a família $X$ no espaço de fase $U=U_{x} \times U_{y} \times U_{\varepsilon} \times U_{\mathcal{A}}$.

Consideremos ao longo da sub variedade $N=\{x=y=\varepsilon=a=b=0\} \subset U$; o Blowing-up

$$
\Phi=\left\{\begin{array}{l}
x=\tau \bar{x} \\
y=\tau^{p} \bar{y} \\
\varepsilon=\tau^{p+1} \bar{\varepsilon} \\
a_{i}=\tau^{2 p-i} \bar{a}_{i}, \quad i=0, \ldots, 2 p-1 \\
b_{j}=\tau^{p-j} \bar{b}_{j}, \quad j=0, \ldots, p-1 \\
\mathcal{A}_{r}=\mathcal{A}_{r}
\end{array}\right.
$$

onde $p$ é a multiplicidade da função $F_{1}(x, 0,0)$ em $x=0$, dado no capítulo 2 , seção 2.3 ; e definamos o campo $\bar{X}$ sobre o aberto

$$
\bar{U}=\Phi^{-1}(U) \in \mathbb{S}^{3 p+2} \times\left(\mathbb{R}^{n-3 p}, 0\right) \times\left(\mathbb{R}^{+}, 0\right) .
$$

(Aqui, $\left(\mathbb{R}^{k}, 0\right)$, representa a família de vizinhanças da origem).

\section{Observação 4.0.5 Notemos que}

- O campo $\left.X\right|_{N}$ se anula em $N$.

- O campo $\bar{X}$ se anula sobre o conjunto $D=\Phi^{-1}(N)=\{\tau=0\} \cap$ $\bar{U}$. (isto decorre diretamente da relação $\bar{X}(\tau)=\tau H(\bar{\varepsilon})$ ) pois $\tau^{p+1} \vec{\varepsilon} e ́$ integral primeira para $\bar{X}$; isto mostra que o conjunto $D$ é invariante por $\bar{X}$, e em particular qualquer fatia

$$
D_{\mathcal{A}_{r}^{0}}=D \cap\left\{\mathcal{A}_{r}=\mathcal{A}_{r}^{0}\right\}
$$


é também invariante por $\bar{X}$.

A família $\left\{\varepsilon, a_{i}, b_{j}, \mathcal{A}_{r}\right\}$ é uma coleção de integrais primeiras para $X$ as quais definem uma folheação 2-dimensional $\mathcal{F}=\left\{d \varepsilon=d a_{i}=d b_{j}=d \mathcal{A}_{r}=0\right\}$, sobre $U \backslash N$. Sobre $\bar{U} \backslash D$ o pull-back de tal folheação é dado por $\overline{\mathcal{F}}=$ $\left\{d\left(\tau^{p+1} \bar{\varepsilon}\right)=d\left(\tau^{2 p-i} \bar{a}_{i}\right)=d\left(\tau^{p-j} \bar{b}_{j}\right)=d \mathcal{A}_{r}=0\right\}$.

O fato relevante é que a folheação $\overline{\mathcal{F}}$ é estendida ao Divisor Excepcional $D$. Esta folheação é definida pelo sistema de equações de Pfaff.

$$
\begin{aligned}
& 0=d(\varepsilon)=(p+1) \tau^{p} \bar{\varepsilon} d(\tau)+\tau^{p+1} d(\bar{\varepsilon}) \\
& 0=d\left(a_{i}\right)=(2 p-i) \tau^{2 p-i-1} \bar{a}_{i} d(\tau)+\tau^{2 p-i} d\left(\bar{a}_{i}\right) \\
& 0=d\left(b_{j}\right)=(p-j) \tau^{p-j-1} \bar{b}_{j} d(\tau)+\tau^{p-j} d\left(\bar{b}_{j}\right) \\
& 0=d\left(\mathcal{A}_{r}\right)
\end{aligned}
$$

Para descrever totalmente esta folheação e a dinâmica do campo $\bar{X}$ sobre $D$, precisaremos de três cartas projetivas que denotaremos por $K_{\bar{\varepsilon}}, K_{ \pm \bar{x}_{\varepsilon}}$ respectivamente.

\subsection{Folheação regular sobre a carta $K_{\bar{\varepsilon}}$} fismo

Sobre o subconjunto $U_{\bar{\varepsilon}}=\{\bar{\varepsilon}>0\} \subset \bar{U}=\Phi^{-1}(U)$; existe um difeomor-

$$
\begin{aligned}
\varphi_{\bar{\varepsilon}}: U_{\bar{\varepsilon}} & \longrightarrow \mathbb{R}^{3 p+2} \times\left(\mathbb{R}^{+}, 0\right) \times\left(\mathbb{R}^{n-3 p}, 0\right) \\
\left((\bar{x}, \bar{y}, \bar{\varepsilon}, \bar{a}, \bar{b}), \mathcal{A}_{r}, \tau\right) & \longmapsto\left((\tilde{x}, \tilde{y}), \tilde{\varepsilon},(A, B), \mathcal{A}_{r}\right)
\end{aligned}
$$

mapeando $D \cap U_{\bar{\varepsilon}}$, sobre $\widetilde{D}=\{\tilde{\varepsilon}=0\} \approx \mathbb{R}^{3 p+2} \times\left(\mathbb{R}^{n-3 p}, 0\right)$, e tal que, o difeomorfismo $\Phi_{\bar{\varepsilon}}=\Phi \circ \varphi_{\bar{\varepsilon}}^{-1}$, é dado por

$$
\Phi_{\bar{\varepsilon}}=\left\{\begin{array}{l}
x=\tilde{\varepsilon} \tilde{x} \\
y=\tilde{\varepsilon}^{p} \tilde{y} \\
\varepsilon=\tilde{\varepsilon}^{p+1} \\
a_{i}=\tilde{\varepsilon}^{2 p-i} A_{i}, \quad i=0, \ldots, 2 p-1 \\
b_{j}=\tilde{\varepsilon}^{p-j} B_{j}, \quad j=0, \ldots, p-1 \\
\mathcal{A}_{r}=\mathcal{A}_{r}
\end{array}\right.
$$

Definiremos a carta $K_{\tilde{\varepsilon}}$ pelas coordenadas $\left(\tilde{x}, \tilde{y}, \tilde{\varepsilon}, A, B, \mathcal{A}_{r}\right)$, dadas por 


$$
\varphi_{\bar{\varepsilon}}=\left\{\begin{array}{lll}
\tilde{x} & =\bar{x} \bar{\varepsilon}^{-\frac{1}{p+1}} \\
\tilde{y} & =\bar{y} \bar{\varepsilon}^{-\frac{p}{p+1}} \\
\tilde{\varepsilon} & =\tau \bar{\varepsilon}^{\frac{1}{p+1}} \\
A_{i}=\bar{a}_{i} \bar{\varepsilon}^{-\frac{2 p-i}{p+1}}, & i=0, \ldots, 2 p-1 \\
B_{j}=\bar{b}_{j} \bar{\varepsilon}^{-\frac{p-j}{p+1}}, \quad j=0, \ldots, p-1 \\
\mathcal{A}_{r}=\mathcal{A}_{r}
\end{array}\right.
$$

A folheação $\overline{\mathcal{F}}$ é descrita nestas coordenadas pelo sistema de Pfaff (restrição à carta $K_{\bar{\varepsilon}}$ )

$$
\begin{aligned}
& 0=d\left(\tilde{\varepsilon}^{p+1}\right)=(p+1) \tilde{\varepsilon}^{p} d(\tilde{\varepsilon}) \\
& 0=d\left(\tilde{\varepsilon}^{2 p-i} A_{i}\right)=(2 p-i) \tilde{\varepsilon}^{2 p-i-1} A_{i} d(\tilde{\varepsilon})+\tilde{\varepsilon}^{2 p-i} d\left(A_{i}\right) \\
& 0=d\left(\tilde{\varepsilon}^{p-j} B_{j}\right)=(p-j) \tilde{\varepsilon}^{p-j-1} B_{i} d(\tilde{\varepsilon})+\tilde{\varepsilon}^{p-j} d\left(B_{j}\right) \\
& 0=d\left(\mathcal{A}_{r}\right)
\end{aligned}
$$

o que é equivalente a $\left\{d \tilde{\varepsilon}=d A_{i}=d B_{j}=d \mathcal{A}_{r}=0\right\}$. Portanto no aberto $U_{\bar{\varepsilon}}$ a folheação $\mathcal{F}$ é regular(ver Figura 4.1).

Como $\bar{X}$ é sempre tangente á folheação $\overline{\mathcal{F}}$, o campo $\left.\bar{X}\right|_{U_{\bar{\varepsilon}}}$ é de fato uma família analítica regular definida na carta $K_{\bar{\varepsilon}}$

$$
X \circ \Phi_{\bar{\varepsilon}}=\left\{\begin{array}{l}
\dot{\tilde{x}}=\widetilde{\varepsilon}^{p} \\
\tilde{\varepsilon}^{p} \dot{\tilde{y}}=\widetilde{F} \\
\dot{\tilde{\varepsilon}}=0 \\
\dot{A}_{i}=0, \quad i=0, \ldots, 2 p-1 \\
\dot{B}_{j}=0, \quad j=0, \ldots, p-1 \\
\dot{\mathcal{A}}_{r}=0
\end{array}\right.
$$

tendo espaço fase $\{(\tilde{x}, \tilde{y})\}$ e parâmetros $\left(\tilde{\varepsilon}, A, B, \mathcal{A}_{r}\right)$ em $\varphi_{\bar{\varepsilon}}\left(U_{\bar{\varepsilon}}\right)$.

Calculemos a expansão $F=F_{0}+F_{1} y+Q y^{2}$ na carta $K_{\bar{\varepsilon}}$.

$$
\begin{aligned}
F_{0}(x, \varepsilon, \mathcal{A}) & =\sum_{i=0}^{2 p-1} a_{i}(\varepsilon, \mathcal{A}) x^{i}+x^{2 p}\left[a_{2 p}(\varepsilon, \mathcal{A})+\sum_{i=2}^{\infty} a_{2 p+i-1}(\varepsilon, \mathcal{A}) x^{i-1}\right] \\
& =\sum_{i=0}^{2 p-1} \tilde{\varepsilon}^{2 p-i} A_{i} \tilde{\varepsilon}^{i} \tilde{x}^{i}+\tilde{\varepsilon}^{2 p} \tilde{x}^{2 p}\left[a_{2 p}(\varepsilon, \mathcal{A})+\tilde{\varepsilon} \sum_{i=2}^{\infty} a_{2 p+i-1}(\varepsilon, \mathcal{A}) \tilde{\varepsilon}^{i-2} \tilde{x}^{i-1}\right] \\
& =\tilde{\varepsilon}^{2 p}\left[\sum_{i=0}^{2 p-1} A_{i} \tilde{x}^{i}+a\left(\mathcal{A}_{r}\right) \tilde{x}^{2 p}+O(\tilde{\varepsilon})\right] \\
& =\tilde{\varepsilon}^{2 p} \widetilde{F}_{0},
\end{aligned}
$$


onde $a_{2 p}(\varepsilon, \mathcal{A})=a\left(\mathcal{A}_{r}\right)+O(\tilde{\varepsilon})$. Similarmente obtemos

$$
\begin{aligned}
F_{1}(x, \varepsilon, \mathcal{A}) & =\sum_{j=0}^{p-1} b_{j}(\varepsilon, \mathcal{A}) x^{j}+x^{p}\left[b_{p}(\varepsilon, \mathcal{A})+\sum_{j=2}^{\infty} b_{p+j-1}(\varepsilon, \mathcal{A}) x^{j-1}\right] \\
& =\sum_{j=0}^{p-1} \tilde{\varepsilon}^{p-j} B_{j} \tilde{\varepsilon}^{j} \tilde{x}^{j}+\tilde{\varepsilon}^{p} \tilde{x}^{p}\left[b_{p}(\varepsilon, \mathcal{A})+\tilde{\varepsilon} \sum_{j=2}^{\infty} b_{p+j-1}(\varepsilon, \mathcal{A}) \tilde{\varepsilon}^{j-2} \tilde{x}^{j-1}\right] \\
& =\tilde{\varepsilon}^{p}\left[\sum_{j=0}^{p-1} B_{j} \tilde{x}^{j}+\mathcal{B}\left(\mathcal{A}_{r}\right) \tilde{x}^{p}+O(\tilde{\varepsilon})\right] \\
& =\tilde{\varepsilon}^{p} \widetilde{F}_{1},
\end{aligned}
$$

onde $b_{p}(\varepsilon, \mathcal{A})=\mathcal{B}\left(\mathcal{A}_{r}\right)+O(\tilde{\varepsilon})$.

Agora considerando a expansão $Q(x, y, \varepsilon, \mathcal{A})=\mathcal{Q}\left(\mathcal{A}_{r}\right)+O(\tilde{\varepsilon})=\widetilde{Q}$, o sistema de equações diferenciais associado será

$$
X \circ \Phi_{\bar{\varepsilon}}=\left\{\begin{array}{l}
\dot{\tilde{x}}=\tilde{\varepsilon}^{p} \\
\dot{\tilde{y}}=\tilde{\varepsilon}^{p}\left[\widetilde{F}_{0}+\widetilde{F}_{1} \tilde{y}+\widetilde{Q} \tilde{y}^{2}\right] \\
\dot{\tilde{\varepsilon}}=0 \\
\dot{A}_{i}=0, \quad i=0, \ldots, 2 p-1 \\
\dot{B}_{j}=0, \quad j=0, \ldots, p-1 \\
\dot{\mathcal{A}_{r}}=0
\end{array}\right.
$$

Daqui fica claro que o campo $\widetilde{X}=\frac{1}{\bar{\varepsilon}^{p}} X \circ \Phi_{\bar{\varepsilon}}$ é dado por

$$
\widetilde{X}=\frac{\partial}{\partial \tilde{x}}+\left[\widetilde{F}_{0}+\widetilde{F}_{1} \tilde{y}+\widetilde{Q} \tilde{y}^{2}\right] \frac{\partial}{\partial \tilde{y}} .
$$

Lembramos que por hipótese $\mathcal{B}_{0}=\mathcal{B}(0) \neq 0, a(0)=0$, e $\mathcal{Q}_{0}=\mathcal{Q}(0)$. Em particular, na fatia $\widetilde{D}_{0}=\widetilde{D} \cap\left\{\mathcal{A}_{r}=0\right\}$ o campo $\widetilde{X}$ é a família de equações diferenciais ordinárias de primeira ordem

$$
R_{A, B}\left(\mathcal{Q}_{0}\right)=\left\{\begin{aligned}
\frac{d \tilde{y}}{d \tilde{x}} & =\widetilde{F}_{0}(\tilde{x}, A)+\widetilde{F}_{1}(\tilde{x}, B) \tilde{y}+\mathcal{Q}_{0} \tilde{y}^{2} \\
& =\left[\sum_{i=0}^{2 p-1} A_{i} \tilde{x}^{i}\right]+\left[\sum_{j=0}^{p-1} B_{j} \tilde{x}^{j}+\mathcal{B}_{0} \tilde{x}^{p}\right] \tilde{y}+\mathcal{Q}_{0} \tilde{y}^{2}
\end{aligned}\right.
$$

Conhecida como uma família Riccati, note que $R_{A, B}(0)$ é uma família de equações lineares. 


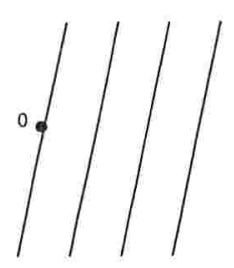

F

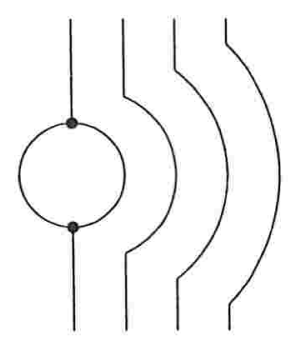

$\tilde{\mathrm{F}}$

Figura 4.1: Folheação Na Carta $K_{\varepsilon}$.

\subsection{Estudo nas cartas $K_{ \pm \bar{x}_{\varepsilon}}$}

Consideremos um subconjunto aberto $U_{ \pm \bar{x}}=\{ \pm \bar{x}>0\} \subset \bar{U}=\Phi^{-1}(U)$ e consideremos o difeomorfismo

$$
\begin{aligned}
\varphi_{ \pm \bar{x}}: \quad U_{ \pm \bar{x}} & \longrightarrow\left(\mathbb{R}^{+}, 0\right) \times \mathbb{R}^{3 p+2} \times\left(\mathbb{R}^{n-3 p}, 0\right) \\
\left((\bar{x}, \bar{y}, \bar{\varepsilon}, \bar{a}, \bar{b}), \mathcal{A}_{r}, \tau\right) & \longmapsto\left(\hat{x},(\hat{y}, \hat{\varepsilon}, \hat{a}, \hat{b}), \mathcal{A}_{r}\right) .
\end{aligned}
$$

Mapeando $D \cap U_{ \pm \bar{x}}$ em $\hat{D}=\{\hat{x}=0\} \approx \mathbb{R}^{3 p+2} \times\left(\mathbb{R}^{n-3 p}, 0\right)$ e tal que o difeomorfismo $\Phi_{ \pm \bar{x}}=\Phi \circ \varphi_{ \pm \bar{x}}^{-1}$, é dado pela correspondência

$$
\Phi_{ \pm \bar{x}}=\left\{\begin{array}{l}
x= \pm \hat{x} \\
y=\hat{x}^{p} \hat{y} \\
\varepsilon=\hat{x}^{p+1} \hat{\varepsilon} \\
a_{i}=\hat{x}^{2 p-i} \hat{a}_{i}, \quad i=0, \ldots, 2 p-1 \\
b_{j}=\hat{x}^{p-j} \hat{b}_{j}, \quad j=0, \ldots, p-1 \\
\mathcal{A}_{r}=\mathcal{A}_{r} .
\end{array}\right.
$$

Aqui as coordenadas $\left(\hat{x},(\hat{y}, \hat{\varepsilon}, \hat{a}, \hat{b}), \mathcal{A}_{r}\right)$, são definidas por

$$
\rho_{ \pm x}=\left\{\begin{array}{l}
\hat{x}= \pm \tau \bar{x} \\
\hat{y}=( \pm)^{p} \bar{x}^{-p} \bar{y} \\
\hat{\varepsilon}=( \pm)^{p+1} \bar{x}^{-(p+1)} \bar{\varepsilon} \\
\hat{a}_{i}=( \pm)^{2 p-i} \bar{x}^{-(2 p-i)} \bar{a}_{i}, \quad i=0, \ldots, 2 p-1 \\
\hat{b}_{j}=( \pm)^{p-j} \bar{x}^{-(p-j)} \bar{b}_{j}, \quad j=0, \ldots, p-1 \\
\mathcal{A}_{r}=\mathcal{A}_{r} .
\end{array}\right.
$$


Nestas coordenadas observemos que a folheação $\overline{\mathcal{F}}$ não é regular, pois é definida pelo seguinte sistema de equações de Pfaff.

$$
\begin{aligned}
& 0=d\left(\hat{x}^{p+1} \hat{\varepsilon}\right)=(p+1) \hat{x}^{p} \hat{\varepsilon} d(\hat{x})+\hat{x}^{p+1} d(\hat{\varepsilon}) \\
& 0=d\left(\hat{x}^{2 p-i} \hat{a}_{i}\right)=(2 p-i) \hat{x}^{2 p-i-1} \hat{a}_{i} d(\hat{x})+\hat{x}^{2 p-i} d\left(\hat{a}_{i}\right) \\
& 0=d\left(\hat{x}^{p-j} \hat{b}_{j}\right)=(p-j) \hat{x}^{p-j-1} \hat{b}_{j} d(\hat{x})+\hat{x}^{p-j} d\left(\hat{b}_{j}\right) \\
& 0=d\left(\mathcal{A}_{r}\right) .
\end{aligned}
$$

Com a finalidade de descrever melhor tal folheação, consideremos o Blowingup

$$
\begin{aligned}
\Psi: \quad \mathbb{R}^{2} \times \mathbb{S}^{3 p} \times\left(\mathbb{R}^{n-3 p}, 0\right) \times \mathbb{R}^{+} & \longrightarrow \mathbb{R}^{2} \times \mathbb{R} \times \mathbb{R}^{3 p} \times \mathbb{R}^{n-3 p} \\
\left((\hat{x}, \hat{y}),\left(\check{e}, \check{\alpha}_{i}, \check{\beta}_{j}\right), \mathcal{A}_{r}, R\right) & \longmapsto\left((\hat{x}, \hat{y}), \hat{\varepsilon}, \hat{a}_{i}, \hat{b}_{j}, \mathcal{A}_{r}\right) .
\end{aligned}
$$

da subvariedade

$$
T=\left\{\hat{\varepsilon}=\hat{a}_{i}=\hat{b}_{j}=0, \quad 0 \leq i \leq 2 p-1, \quad 0 \leq j \leq p-1\right\}
$$

definido pela expressão

$$
\Psi= \begin{cases}\left(\hat{x}, \hat{y}, \mathcal{A}_{r}\right) & =\left(\hat{x}, \hat{y}, \mathcal{A}_{r}\right) \\ \hat{\varepsilon} & =R^{p+1} \check{e} \\ \hat{a}_{i} & =R^{2 p-i} \check{\alpha}_{i}, \quad i=0, \ldots, 2 p-1 \\ \hat{b}_{j} & =R^{p-j} \check{\beta}_{j}, \quad j=0, \ldots, p-1\end{cases}
$$

Sobre o subconjunto aberto $U_{\check{e}}=\{\check{e}>0\} \subset \check{U}=\Psi^{-1}\left(\varphi_{ \pm \bar{x}}\left(U_{ \pm \bar{x}}\right)\right)$, consideremos o difeomorfismo

$$
\begin{aligned}
\varphi_{\check{e}}: \quad U_{\check{e}} & \longrightarrow \mathbb{R}^{2} \times\left(\mathbb{R}^{+}, 0\right) \times\left(\mathbb{R}^{3 p}, 0\right) \times\left(\mathbb{R}^{n-3 p}, 0\right) \\
\left((\hat{x}, \hat{y}),\left(\check{e}, \check{\alpha}_{i}, \check{\beta}_{j}\right), \mathcal{A}_{r}\right) & \longmapsto\left((\hat{x}, \hat{y}), e,\left(A_{i}, B_{j}\right), \mathcal{A}_{r}\right) .
\end{aligned}
$$

Mapeamos $D=\{R=0\}$ em $\check{D}=\{e=0\}$, e tal que o difeomorfismo $\Psi_{\tilde{e}}=\Psi \circ \varphi_{\tilde{e}}^{-1}$ é dado por

$$
\Psi_{\check{e}}= \begin{cases}\left(\hat{x}, \hat{y}, \mathcal{A}_{r}\right) & =\left(\hat{x}, \hat{y}, \mathcal{A}_{r}\right) \\ \hat{\varepsilon} & =e^{p+1} \\ \hat{a}_{i} & =e^{2 p-i} A_{i}, \quad i=0, \ldots, 2 p-1 . \\ \hat{b}_{j} & =e^{p-j} B_{j}, \quad j=0, \ldots, p-1 .\end{cases}
$$

Chamaremos as coordenadas $\left(\hat{x}, \hat{y}, e, A, B, \mathcal{A}_{r}\right)$ de cartas $K_{ \pm \bar{x}_{\varepsilon}}$ do Blowingup, que são dadas por

$$
\varphi_{\dot{e}}= \begin{cases}\left(\hat{x}, \hat{y}, \mathcal{A}_{r}\right) & =\left(\hat{x}, \hat{y}, \mathcal{A}_{r}\right) \\ e & =\operatorname{Re} \frac{1}{p+1} \\ A_{i} & =\check{e}^{-\frac{2 p-i}{p+1}} \check{\alpha}_{i}, \quad i=0, \ldots, 2 p-1 . \\ B_{j} & =\check{e}^{-\frac{p-j}{p+1}} \check{\zeta}_{j}, \quad j=0, \ldots, p-1 .\end{cases}
$$


Assim obtemos o seguinte difeomorfismo

$$
\begin{aligned}
\Phi_{ \pm \bar{x}_{\epsilon}} & =\Phi \circ\left(\varphi_{ \pm \bar{x}}^{-1} \circ \Psi \circ \varphi_{\check{e}}^{-1}\right) \\
& =\Phi_{ \pm \bar{x}} \circ \Psi_{\check{e}}
\end{aligned}
$$

dado pela correspondência

$$
\Phi_{ \pm \bar{x}_{\varepsilon}}=\left\{\begin{array}{l}
x= \pm \hat{x} \\
y=\hat{x}^{p} \hat{y} \\
\varepsilon=\hat{x}^{p+1} e^{p+1} \\
a_{i}=\hat{x}^{2 p-i} e^{2 p-i} A_{i}, \quad i=0, \ldots, 2 p-1 . \\
b_{j}=\hat{x}^{p-j} e^{p-j} B_{j}, \quad j=0, \ldots, p-1 . \\
\mathcal{A}_{r}=\mathcal{A}_{r}
\end{array}\right.
$$

Nesta carta, a folheação $\overline{\mathcal{F}}$ é dada pelo sistema de Pfaff.

$$
\begin{aligned}
& 0=d\left(\hat{x}^{p+1} e^{p+1}\right)=(p+1)(\hat{x} e)^{p} d(\hat{x} e) \\
& 0=d\left(\hat{x}^{2 p-i} e^{2 p-i} A_{i}\right)=(2 p-i)(\hat{x} e)^{2 p-i-1} A_{i} d(\hat{x} e)+\hat{x}^{2 p-i} e^{2 p-i} d\left(A_{i}\right) \\
& 0=d\left(\hat{x}^{p-j} e^{p-j} B_{j}\right)=(p-j)(\hat{x} e)^{p-j-1} B_{j} d(\hat{x} e)+\hat{x}^{p-j} e^{p-j} d\left(B_{j}\right) \\
& 0=d\left(\mathcal{A}_{r}\right)
\end{aligned}
$$

Que é equivalente a $\left\{d(\hat{x} e)=d A_{i}=d B_{j}=d \mathcal{A}_{r}=0\right\}$

\section{Observação 4.2.1 Notemos que}

- Para cada $\mathcal{A}^{0}=\left(A^{0}, B^{0}, \mathcal{A}_{r}^{0}\right) \in U_{\mathcal{A}}$, a folheação $\hat{\mathcal{F}}$ é definida pelos niveis da função $(\hat{x}, e) \longmapsto \hat{x} e$. Assim a folheação

$$
\hat{\mathcal{F}}_{\hat{x} e}=\hat{\mathcal{F}} \cup E_{\hat{x}} \cup E_{e}
$$

constitui a extensão de $\hat{\mathcal{F}}$ ao divisor excepcional $\hat{D} \cup \check{D}$, onde

$$
\begin{aligned}
& E_{\hat{x}}=\hat{D} \cap\left\{\mathcal{A}=\mathcal{A}^{0}\right\} \\
& E_{e}=\check{D} \cap\left\{\mathcal{A}=\mathcal{A}^{0}\right\}
\end{aligned}
$$

são sub variedades 2-dimensionais parametrizadas pelas coordenadas $\left(\hat{y}, e, \mathcal{A}^{0}\right),\left(\hat{x}, \hat{y}, \mathcal{A}^{0}\right)$, respectivamente.

- A folha limite $E_{\hat{x}} \cup E_{e}$ é uma folha singular da função $(\hat{x}, e) \longmapsto \hat{x} e$ 
Nas cartas $K_{ \pm \bar{x}_{\varepsilon}}$, o campo $\left.\bar{X}\right|_{U_{ \pm \bar{x}}}$ é dado por

$$
\begin{aligned}
\bar{X}^{ \pm}:=\left.\bar{X}\right|_{U_{ \pm \bar{x}}} & =\left(\frac{1}{\hat{x}^{p}}\right) X \circ \Phi \circ\left(\varphi_{ \pm \bar{x}}^{-1} \circ \Psi \circ \varphi_{\tilde{e}}^{-1}\right) \\
& =\left(\frac{1}{\hat{x}^{p}}\right) X \circ\left(\Phi \circ \varphi_{ \pm \bar{x}}^{-1}\right) \circ\left(\Psi \circ \varphi_{\breve{e}}^{-1}\right) \\
& =\left(\frac{1}{\hat{x}^{p}}\right) X \circ\left(\Phi_{ \pm \bar{x}} \circ \Psi_{\check{e}}\right) \\
& =\left(\frac{1}{\hat{x}^{p}}\right) X \circ \Phi_{ \pm \bar{x}_{\varepsilon}},
\end{aligned}
$$

onde

$$
X \circ \Phi_{ \pm \bar{x}_{\varepsilon}}=\left\{\begin{array}{l}
\dot{\hat{x}}= \pm \hat{x}^{p+1} e^{p+1} \\
\hat{x}^{p}=\hat{F}^{ \pm} \mp p \hat{x}^{2 p} e^{p+1} \hat{y} \\
\dot{e}=\mp \hat{x}^{p} e^{p+2} \\
\dot{A}_{i}=0, \quad i=0, \ldots, 2 p-1 . \\
\dot{B}_{j}=0, \quad j=0, \ldots, p-1 . \\
\dot{\mathcal{A}}_{r}=0
\end{array}\right.
$$

Agora observemos na carta $K_{ \pm \bar{x}_{\varepsilon}}$, a expansão $F=F_{0}+F_{1} y+Q y^{2}$.

$$
\begin{aligned}
F_{0}(x, \varepsilon, \mathcal{A})= & \sum_{i=0}^{2 p-1} a_{i}(\varepsilon, \mathcal{A}) x^{i}+x^{2 p}\left[a_{2 p}(\varepsilon, \mathcal{A})+\sum_{i=2}^{\infty} a_{2 p+i-1}(\varepsilon, \mathcal{A}) x^{i-1}\right] \\
= & \sum_{i=0}^{2 p-1} \hat{x}^{2 p-i} e^{2 p-i} A_{i}( \pm \hat{x})^{i}+ \\
& +( \pm \hat{x})^{2 p}\left[a_{2 p}(\varepsilon, \mathcal{A})+\hat{x} \sum_{i=2}^{\infty} a_{2 p+i-1}(\varepsilon, \mathcal{A}) \hat{x}^{i-2}( \pm 1)^{i-1}\right] \\
= & \hat{x}^{2 p}\left[\sum_{i=0}^{2 p-1}( \pm 1)^{i} A_{i} e^{2 p-i}+a\left(\mathcal{A}_{r}\right)+O(\hat{x})\right] \\
= & \hat{x}^{2 p} \hat{F}_{0}^{ \pm}
\end{aligned}
$$


onde $a_{2 p}(\varepsilon, \mathcal{A})=a\left(\mathcal{A}_{r}\right)+O(\hat{x})$. Similarmente obtemos

$$
\begin{aligned}
F_{1}(x, \varepsilon, \mathcal{A})= & \sum_{j=0}^{p-1} b_{j}(\varepsilon, \mathcal{A}) x^{j}+x^{p}\left[b_{p}(\varepsilon, \mathcal{A})+\sum_{j=2}^{\infty} b_{p+j-1}(\varepsilon, \mathcal{A}) x^{j-1}\right] \\
= & \sum_{j=0}^{p-1} \hat{x}^{p-j} e^{p-j} B_{j}( \pm \hat{x})^{j}+ \\
& +( \pm \hat{x})^{p}\left[b_{p}(\varepsilon, \mathcal{A})+\hat{x} \sum_{j=2}^{\infty} b_{p+j-1}(\varepsilon, \mathcal{A})( \pm 1)^{j-1} \hat{x}^{j-2}\right] \\
= & \hat{x}^{p}\left[\sum_{j=0}^{p-1}( \pm 1)^{j} B_{j} e^{p-j}+( \pm 1)^{p} \mathcal{B}\left(\mathcal{A}_{r}\right)+O(\hat{x})\right] \\
= & \hat{x}^{p} \hat{F}_{1}^{ \pm}
\end{aligned}
$$

onde $b_{p}(\varepsilon, \mathcal{A})=\mathcal{B}\left(\mathcal{A}_{r}\right)+O(\hat{x})$.

Da mesma forma, $Q(x, y, \varepsilon, \mathcal{A})=\mathcal{Q}\left(\mathcal{A}_{r}\right)+O(\hat{x})=\hat{Q}^{ \pm}$. Assim, obtemos a expressão

$$
\hat{F}^{ \pm}=\hat{x}^{2 p}\left[\hat{F}_{0}^{ \pm}+\hat{F}_{1}^{ \pm} \hat{y}+\hat{Q}^{ \pm} \hat{y}^{2}\right]
$$

Logo, o sistema de equações diferenciais associado a $X \circ \Phi_{ \pm \bar{x}_{\varepsilon}}$ será

$$
X \circ \Phi_{ \pm \bar{x}_{\varepsilon}}=\left\{\begin{array}{l}
\dot{\hat{x}}= \pm \hat{x}^{p+1} e^{p+1} \\
\dot{\hat{y}}=\hat{x}^{p}\left[\hat{F}_{0}^{ \pm}+\left(\mp p e^{p+1}+\hat{F}_{1}^{ \pm}\right) \hat{y}+\hat{Q}^{ \pm} \hat{y}^{2}\right] \\
\dot{e}=\mp \hat{x}^{p} e^{p+2} \\
\dot{A}_{i}=0, \quad i=0, \ldots, 2 p-1 . \\
\dot{B}_{j}=0, \quad j=0, \ldots, p-1 . \\
\dot{\mathcal{A}}_{r}=0
\end{array}\right.
$$

Portanto, nas cartas $K_{ \pm \bar{x}_{\varepsilon}}$, obtemos a família de campos vetoriais $\bar{X}^{ \pm}$com espaço fase $(\hat{x}, \hat{y}, e)$ e parâmetros $\left(A, B, \mathcal{A}_{r}\right)$ dado por:

$$
\begin{aligned}
\bar{X}^{ \pm} & :=\left( \pm \hat{x} e^{p+1}\right) \frac{\partial}{\partial \hat{x}}+\bar{F}^{ \pm}\left(\hat{x}, \hat{y}, e, A, B, \mathcal{A}_{r}\right) \frac{\partial}{\partial \hat{y}}+\left(\mp e^{p+2}\right) \frac{\partial}{\partial e} \\
& = \pm e^{p+1}\left(\hat{x} \frac{\partial}{\partial \hat{x}}-e \frac{\partial}{\partial e}\right)+\bar{F}^{ \pm}\left(\hat{x}, \hat{y}, e, A, B, \mathcal{A}_{r}\right) \frac{\partial}{\partial \hat{y}},
\end{aligned}
$$

onde $\quad \bar{F}^{ \pm}=\hat{F}_{0}^{ \pm}+\left(\mp p e^{p+1}+\hat{F}_{1}^{ \pm}\right) \hat{y}+\hat{Q}^{ \pm} \hat{y}^{2}$

Observação 4.2.2 Olhando para o campo $\bar{X}^{ \pm}$, note que 
A função $f:(\hat{x}, e) \longmapsto \hat{x}$ e, é claramente uma integral primeira para $\bar{X}^{ \pm}$ pois verifica $\nabla f \cdot \bar{X}^{ \pm}=\bar{X}^{ \pm}(f) \equiv 0$; ou seja a função $f$ é constante ao longo das curvas integrais de $\bar{X}^{ \pm}$. Assim $\bar{X}^{ \pm}$é sempre tangente á folheação $\hat{\mathcal{F}}_{\hat{x} e}=$ $\hat{\mathcal{F}} \cup E_{\hat{x}} \cup E_{e}$ e portanto tangente ao divisor excepcional $\hat{D}=\{\hat{x}=0\}$ (ver Figura 4.2).

Em particular, tomando $Q(0)=Q_{0}, a(0)=0, \mathcal{B}(0)=\mathcal{B}_{0} ;$ sobre o conjunto $\hat{D}_{0}=\hat{D} \cap\left\{\mathcal{A}_{r}=0\right\}$ temos a familia restrita

$$
\left.\bar{X}^{ \pm}\right|_{\hat{D}_{0}}:=\mp e^{p+2} \frac{\partial}{\partial e}+\bar{F}^{ \pm}(0, \hat{y}, e, A, B, 0) \frac{\partial}{\partial \hat{y}},
$$

onde

$$
\begin{aligned}
\bar{F}^{ \pm}(0, \hat{y}, e, A, B, 0) & =\hat{F}_{0}^{ \pm}(e, A)+\left(\mp p e^{p+1}+\hat{F}_{1}^{ \pm}(e, B)\right) \hat{y}+Q_{0} \hat{y}^{2} \\
\hat{F}_{0}^{ \pm}(e, A) & =\sum_{i=0}^{2 p-1}( \pm 1)^{i} A_{i} e^{2 p-i} \\
\hat{F}_{1}^{ \pm}(e, B) & =\sum_{j=0}^{p-1}( \pm 1)^{j} B_{j} e^{p-j}+( \pm 1)^{p} \mathcal{B}_{0},
\end{aligned}
$$

Aqui o espaço fase $(\hat{y}, e)$ corresponderia à família de equações diferenciais ordinárias de primeira ordem

$$
C_{A, B}^{ \pm}\left(\mathcal{Q}_{0}\right)=\left\{\begin{aligned}
\frac{d \hat{y}}{d e}=\frac{1}{\mp e^{p+2}} & \left(\left[\sum_{i=0}^{2 p-1}( \pm 1)^{i} A_{i} e^{2 p-i}\right]+\left[\mp p e^{p+1}+\right.\right. \\
& \left.\left.+\sum_{j=0}^{p-1}( \pm 1)^{j} B_{j} e^{p-j}+( \pm 1)^{p} \mathcal{B}_{0}\right] \hat{y}+\mathcal{Q}_{0} \hat{y}^{2}\right)
\end{aligned}\right.
$$

onde $(A, B)=\left(A_{0}, \ldots, A_{2 p-1}, B_{0}, \ldots, B_{p-1}\right)$

\subsection{Relação entre as cartas $K_{\bar{\varepsilon}}, K_{ \pm} \bar{x}_{\varepsilon}$}

Lembremos que as cartas $K_{ \pm \bar{x}_{\varepsilon}}$ e $K_{\bar{\varepsilon}}$ estão definidas respectivamente pelas seguintes coordenadas:

$$
\Phi_{ \pm \bar{x}_{\varepsilon}}=\left\{\begin{array}{l}
x= \pm \hat{x} \\
y=\hat{x}^{p} \hat{y} \\
\varepsilon=\hat{x}^{p+1} e^{p+1} \\
a_{i}=\hat{x}^{2 p-i} e^{2 p-i} A_{i} \\
b_{j}=\hat{x}^{p-j} e^{p-j} B_{j} \\
\mathcal{A}_{r}=\mathcal{A}_{r}
\end{array} \quad \Phi_{\bar{\varepsilon}}=\left\{\begin{array}{l}
x=\tilde{\varepsilon} \tilde{x} \\
y=\tilde{\varepsilon}^{p} \tilde{y} \\
\varepsilon=\tilde{\varepsilon}^{p+1} \\
a_{i}=\tilde{\varepsilon}^{2 p-i} A_{i} \\
b_{j}=\tilde{\varepsilon}^{p-j} B_{j} \\
\mathcal{A}_{r}=\mathcal{A}_{r},
\end{array}\right.\right.
$$


4.3. RELAÇÃO ENTRE AS CARTAS $K_{\bar{\varepsilon}}, K_{ \pm} \bar{X}_{\bar{\varepsilon}}$

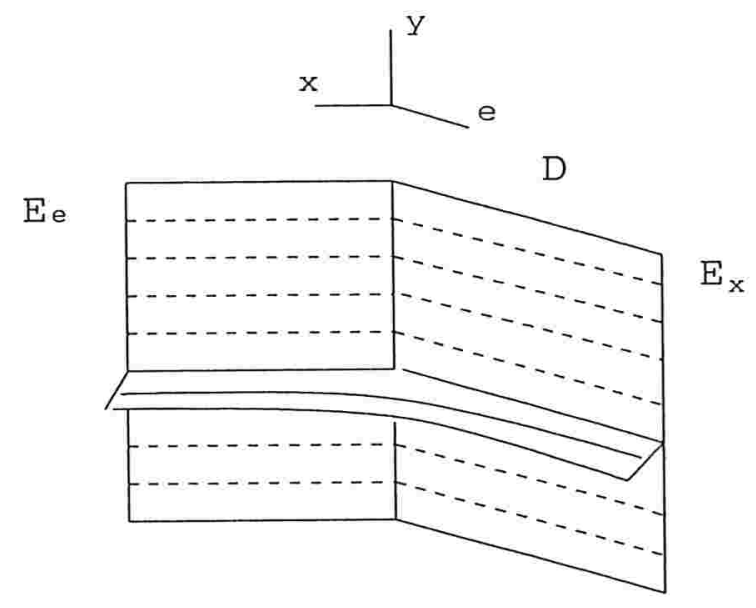

Figura 4.2: Folheação Na Carta $K_{-x_{\varepsilon}}$.

onde $\quad 0 \leq i \leq 2 p-1,0 \leq j \leq p-1$.

Consideremos o seguinte difeomorfismo

$$
\begin{aligned}
D_{\varepsilon}^{ \pm}:=D\left(K_{\bar{\varepsilon}}, K_{ \pm \bar{x}_{\varepsilon}}\right) & =\varphi_{\bar{\varepsilon}} \circ \varphi_{ \pm \bar{x}}^{-1} \circ \Psi \circ \varphi_{\check{e}}^{-1} \\
& =\left(\Phi \circ \varphi_{\bar{\varepsilon}}^{-1}\right)^{-1} \circ\left(\Phi \circ \varphi_{ \pm \bar{x}}^{-1}\right) \circ\left(\Psi \circ \varphi_{\check{e}}^{-1}\right) \\
& =\left(\Phi_{\bar{\varepsilon}}\right)^{-1} \circ\left(\Phi_{ \pm \bar{x}} \circ \Psi_{\check{e}}\right) \\
& =\left(\Phi_{\bar{\varepsilon}}\right)^{-1} \circ \Phi_{ \pm \bar{x}_{\bar{\varepsilon}}}
\end{aligned}
$$

Onde

$$
\Phi_{\bar{\varepsilon}}^{-1}=\left\{\begin{array}{l}
\tilde{x}=\frac{x}{\tilde{\varepsilon}} \\
\tilde{y}=\frac{y}{\tilde{\varepsilon}^{p}} \\
\tilde{\varepsilon}=\frac{1}{\bar{\varepsilon}^{p+1}} \\
A_{i}=\frac{a_{i}}{\bar{\varepsilon}^{2 p-i}} \\
B_{j}=\frac{b_{j}}{\bar{\varepsilon}^{p-j}} \\
\mathcal{A}_{r}=\mathcal{A}_{r},
\end{array}\right.
$$

Logo os difeomorfismos $D_{\varepsilon}^{ \pm}$, e $\left(D_{\varepsilon}^{ \pm}\right)^{-1}$ são dados pelas seguintes correspondências

$$
D_{\varepsilon}^{ \pm}=\left\{\begin{array}{lll}
\tilde{x} & =\frac{ \pm 1}{e} \\
\tilde{y} & =\frac{\tilde{y}}{e^{p}} \\
\tilde{\varepsilon} & =\hat{x} e \\
\left(A_{i}, B_{j}, \mathcal{A}_{r}\right) & =\left(A_{i}, B_{j}, \mathcal{A}_{r}\right)
\end{array} \quad\left(D_{\varepsilon}^{ \pm}\right)^{-1}= \begin{cases}\hat{x} & = \pm \tilde{\varepsilon} \tilde{x} \\
\hat{y} & =( \pm 1)^{p} \frac{\bar{y}}{\bar{x}^{p}} \\
e & =\frac{ \pm 1}{\bar{x}} \\
\left(A_{i}, B_{j}, \mathcal{A}_{r}\right) & =\left(A_{i}, B_{j}, \mathcal{A}_{r}\right)\end{cases}\right.
$$


Observação 4.3.1 Destas relações temos que

- Claramente os difeomorfismos $D_{\varepsilon}^{ \pm} e\left(D_{\varepsilon}^{ \pm}\right)^{-1}$ relacionam as coordena$\operatorname{das}\left(\hat{x}, \hat{y}, e, A, B, \mathcal{A}_{r}\right),\left(\tilde{x}, \tilde{y}, \tilde{\varepsilon}, A, B, \mathcal{A}_{r}\right)$ que definem as cartas $K_{ \pm \bar{x}_{\varepsilon}}, K_{\bar{\varepsilon}}$.

- Os mesmos parâmetros $\left(A, B, \mathcal{A}_{r}\right)$ correspondem para ambas familias $\widetilde{X}$ e $\bar{X}^{ \pm}$; portanto também para ambas famılias $R_{A, B}\left(\mathcal{Q}_{0}\right)$ e $C_{A, B}^{ \pm}\left(\mathcal{Q}_{0}\right)$.

Isso mostra que a família $\left.C_{A, B}^{ \pm}\left(\mathcal{Q}_{0}\right) \equiv \bar{X}^{ \pm}\right|_{\hat{D}_{0}}$, pode ser vista como uma compactificação da família Riccati $\left.R_{A, B}\left(\mathcal{Q}_{0}\right) \equiv \tilde{X}\right|_{\tilde{D}_{0}}$ em $\tilde{x}= \pm \infty$. 


\section{Capítulo 5}

\section{Dinâmica Assintótica sobre a carta $K_{\bar{\varepsilon}}$}

Após a desingularização, os campos vetoriais $\tilde{X}, \bar{X}^{ \pm}$, se correspondem pelas funções $D_{\varepsilon}^{ \pm}$(definidas no capítulo anterior). Sobre divisor $\tilde{D}_{0}=\{\tilde{\varepsilon}=$ $0\} \cap\left\{\mathcal{A}_{r}=0\right\}$, vamos caracterizar o comportamento assintótico das soluções $\tilde{y}(\tilde{x})$ da família $R_{A, B}\left(\mathcal{Q}_{0}\right)$ numa vizinhança de $\tilde{x}= \pm \infty$. Este comportamento assintótico é equivalente (devido ao difeomorfismo $\left(D_{\varepsilon}^{ \pm}\right)^{-1}$ ) a estudar as soluções de $C_{A, B}^{ \pm}\left(\mathcal{Q}_{0}\right)$ numa vizinhança de $X=0$ com espaço fase $\left(X=\frac{ \pm 1}{\tilde{x}}, Y=( \pm 1)^{p} \frac{\tilde{y}}{\tilde{x}^{p}}\right)$ e parâmetros $(A, B)$.

A caracterização consistirá em definir adequadamente a condição inicial $\tilde{y}_{0}$, tal que o problema de Cauchy $\left[R_{A, B}\left(\mathcal{Q}_{0}\right), \tilde{y}(0)=\tilde{y}_{0}\right]$ tenha solução $\tilde{y}(\tilde{x})$, satisfazendo $\tilde{y}(\tilde{x})=o\left(\tilde{x}^{p}\right)$ ou equivalentemente $Y(X)=o(1)$, sobre vizinhanças de $\tilde{x}= \pm \infty$ e $X=0$ respectivamente.

\subsection{O caso $R_{A, 0}(0)$}

Vamos começar com o caso especial da família $R_{A, B}\left(\mathcal{Q}_{0}\right)$ com $B=0=$ $\mathcal{Q}_{0}$, neste caso consideremos a família linear de equações diferenciais

$$
\frac{d \tilde{y}}{d \tilde{x}}=\left(\sum_{k=0}^{2 p-1} A_{k} \tilde{x}^{k}\right)+\left(\mathcal{B}_{0} \tilde{x}^{p}\right) \tilde{y}
$$

onde $A=\left(A_{0}, \ldots, A_{2 p-1}\right), \quad \mathcal{B}_{0} \neq 0$.

Pela fórmula de variação das constantes, o problema de Cauchy associado 
com condição inicial $\tilde{y}_{0}$ tem uma única solução sobre $\mathbb{R}$ dada por

$$
\tilde{y}(\tilde{x})=e^{\mathcal{B}_{0} \frac{\tilde{x}^{p+1}}{p+1}}\left[\tilde{y}_{0}+\sum_{k=0}^{2 p-1} A_{k} J_{k}(\tilde{x})\right]
$$

Onde, $\quad J_{k}(\tilde{x})=\int_{0}^{\bar{x}} \tau^{k} e^{-\mathcal{B}_{0} \frac{\tau^{p+1}}{p+1}} d \tau, \quad k=0,1, \ldots, 2 p-1$.

Notemos que o comportamento assintótico destas soluções numa vizinhança de $\tilde{x}= \pm \infty$ depende da expansão assintótica das integrais $J_{k}(\tilde{x})$ quando $\tilde{x} \rightarrow \pm \infty$, conseqüentemente dependerá das constantes $\mathcal{B}_{0}$ e $p+1$.

\subsubsection{Caso $\mathcal{B}_{0}>0$}

Como as funções $J_{k}(\tilde{x})=\int_{0}^{\tilde{x}} \tau^{k} e^{-\mathcal{B}_{0} \frac{\tau^{p+1}}{p+1}} d \tau$ são crescentes e limitadas para todo $k=0,1, \ldots, 2 p-1$, então vale a igualdade

$$
J_{k}(\tilde{x})=\int_{0}^{\infty} \tau^{k} e^{-\mathcal{B}_{0} \frac{\tau^{p+1}}{p+1}} d \tau-\int_{\tilde{x}}^{\infty} \tau^{k} e^{-\mathcal{B}_{0} \frac{\tau^{p+1}}{p+1}} d \tau .
$$

Da proposição 7.4.9 e o exemplo 7.4.6(ver apêndice 1), valem as seguintes expressões

$$
\begin{aligned}
c_{k}\left(\mathcal{B}_{0}\right):= & \int_{0}^{\infty} \tau^{k} e^{-\mathcal{B}_{0} \frac{\tau^{p+1}}{p+1}} d \tau=\mathcal{B}_{0}^{-\frac{k+1}{p+1}}(p+1)^{\frac{k+1}{p+1}-1} \Gamma\left(\frac{k+1}{p+1}\right) \\
\int_{\tilde{x}}^{\infty} \tau^{k} e^{-\mathcal{B}_{0} \frac{\tau^{p+1}}{p+1}} d \tau & =\frac{1}{\mathcal{B}_{0}} \tilde{x}^{k-p} e^{-\mathcal{B}_{0} \frac{\dot{x}^{p+1}}{p+1}}+o\left(\tilde{x}^{k-p} e^{-\mathcal{B}_{0} \frac{\bar{x}^{p+1}}{p+1}}\right)
\end{aligned}
$$

onde, $\Gamma(x)=\int_{0}^{\infty} \tau^{x-1} e^{-\tau} d \tau$ é convergente para $x>0$.

Assim quando $\tilde{x} \rightarrow+\infty, J_{k}(\tilde{x})$ tem a seguinte expansão

$$
J_{k}(\tilde{x})=c_{k}\left(\mathcal{B}_{0}\right)+\left(\frac{-1}{\mathcal{B}_{0}}\right) \tilde{x}^{k-p} e^{-\mathcal{B}_{0} \frac{\tilde{x}^{p+1}}{p+1}}+o\left(\tilde{x}^{k-p} e^{-\mathcal{B}_{0} \frac{\tilde{x}^{p+1}}{p+1}}\right)
$$

Logo, temos o resultado

Lema 5.1.1 As soluções $\tilde{y}_{ \pm}(\tilde{x})$ numa vizinhança de $\tilde{x}= \pm \infty$ do problema de Cauchy $\left[R_{A, 0}(0), \tilde{y}_{0}\right]$, satisfazem o seguinte

(i) $\tilde{y}_{+}(\tilde{x})=o\left(\tilde{x}^{p}\right)$, numa vizinhança de $\tilde{x}=+\infty$ se e só se

$$
\tilde{y}_{0}+\sum_{k=0}^{2 p-1} c_{k}\left(\mathcal{B}_{0}\right) A_{k}=0
$$

onde $\tilde{y}_{0}:=\tilde{y}_{+}(0)$. 
(ii) Se $p+1$ é par, $\tilde{y}_{-}(\tilde{x})=o\left(\tilde{x}^{p}\right)$ numa vizinhança de $\tilde{x}=-\infty$, se e só mente se

$$
\tilde{y}_{0}+\sum_{k=0}^{2 p-1}(-1)^{k+1} c_{k}\left(\mathcal{B}_{0}\right) A_{k}=0
$$

onde $\tilde{y}_{0}:=\tilde{y}_{-}(0)$.

(iii) Se $p+1$ é ímpar, $\tilde{y}_{-}(\tilde{x})=o\left(\tilde{x}^{p}\right)$ numa vizinhança de $\tilde{x}=-\infty$, para qualquer vetor $\left(\tilde{y}_{0}, A\right):=\left(\tilde{y}_{-}(0), A_{0}, \ldots, A_{2 p-1}\right)$.

\section{DEMONSTRAÇÃO}

(i) Substituindo a expressão (5.2) na solução $\tilde{y}_{+}(\tilde{x})$ dada em (5.1), resulta a igualdade

$$
\begin{aligned}
\tilde{y}_{+}(\tilde{x})= & e^{\mathcal{B}_{0} \frac{\tilde{x}^{p+1}}{p+1}}\left[\tilde{y}_{0}+\sum_{k=0}^{2 p-1} c_{k}\left(\mathcal{B}_{0}\right) A_{k}\right]+\left(\frac{-1}{\mathcal{B}_{0}}\right) \sum_{k=0}^{2 p-1} A_{k} \tilde{x}^{k-p}+ \\
& +\sum_{k=0}^{2 p-1} o\left(\tilde{x}^{k-p}\right)
\end{aligned}
$$

de onde obtemos

$$
\begin{aligned}
\frac{\tilde{y}_{+}(\tilde{x})}{\tilde{x}^{p}}= & \frac{1}{\tilde{x}^{p}} e^{\mathcal{B}_{0} \frac{\tilde{x}^{p+1}}{p+1}}\left[\tilde{y}_{0}+\sum_{k=0}^{2 p-1} c_{k}\left(\mathcal{B}_{0}\right) A_{k}\right]+\left(\frac{-1}{\mathcal{B}_{0}}\right) \sum_{k=0}^{2 p-1} A_{k} \tilde{x}^{k-2 p}+ \\
& +\sum_{k=0}^{2 p-1} x^{k-2 p} \frac{o\left(\tilde{x}^{k-p}\right)}{x^{k-p}} .
\end{aligned}
$$

Daqui decorre a condição necessária e suficiente, mostrando (i).

(ii) Se $p+1$ é par, vale a relação

$$
\begin{aligned}
J_{k}(-\tilde{x})= & (-1)^{k+1} J_{k}(\tilde{x}) \\
= & (-1)^{k+1}\left[c_{k}\left(\mathcal{B}_{0}\right)+\left(\frac{-1}{\mathcal{B}_{0}}\right) \tilde{x}^{k-p} e^{-\mathcal{B}_{0} \frac{\tilde{x}^{p+1}}{p+1}}+\right. \\
& \left.+o\left(\tilde{x}^{k-p} e^{-\mathcal{B}_{0} \frac{\dot{x}^{p+1}}{p+1}}\right)\right]
\end{aligned}
$$


Logo daqui, definindo $\tilde{y}_{-}(\tilde{x}):=\tilde{y}(-\tilde{x})$, e usando (5.1) obtemos em forma análoga a seguinte expressão:

$$
\begin{aligned}
\frac{\tilde{y}_{-}(\tilde{x})}{\tilde{x}^{p}}= & \frac{1}{\tilde{x}^{p}} e^{\mathcal{B}_{0} \frac{\tilde{x}^{p+1}}{p+1}}\left[\tilde{y}_{0}+\sum_{k=0}^{2 p-1}(-1)^{k+1} c_{k}\left(\mathcal{B}_{0}\right) A_{k}\right]+ \\
& +\left(\frac{-1}{\mathcal{B}_{0}}\right) \sum_{k=0}^{2 p-1}(-1)^{k+1} A_{k} \tilde{x}^{k-2 p}+\sum_{k=0}^{2 p-1} x^{k-2 p} \frac{o\left(\tilde{x}^{k-p}\right)}{x^{k-p}}
\end{aligned}
$$

onde claramente $\tilde{y}_{0}:=\tilde{y}_{-}(0)$. Assim, obtemos a condição necessária e suficiente (ii).

(iii) Se $p+1$ fosse ímpar, obteríamos

$$
\begin{aligned}
J_{k}(-\tilde{x}) & =(-1)^{k+1} \int_{0}^{\bar{x}} \tau^{k} e^{\mathcal{B}_{0} \frac{\tau^{p+1}}{p+1}} d \tau \\
& =(-1)^{k+1}\left[\left(\frac{1}{\mathcal{B}_{0}}\right) \tilde{x}^{k-p} e^{\mathcal{B}_{0} \frac{\bar{x}^{p+1}}{p+1}}+o\left(\tilde{x}^{k-p} e^{\mathcal{B}_{0} \frac{\hat{x}^{p+1}}{p+1}}\right)\right] .
\end{aligned}
$$

Definindo $\tilde{y}_{-}(\tilde{x}):=\tilde{y}(-\tilde{x})$, e usando (5.1), obtemos

$$
\begin{aligned}
\frac{\tilde{y}_{-}(\tilde{x})}{\tilde{x}^{p}}= & \frac{1}{\tilde{x}^{p}} e^{-\mathcal{B}_{0} \frac{\tilde{x}^{p+1}}{p+1}} \tilde{y}_{0}+\left(\frac{1}{\mathcal{B}_{0}}\right) \sum_{k=0}^{2 p-1}(-1)^{k+1} A_{k} \tilde{x}^{k-2 p} \\
& +\sum_{k=0}^{2 p-1} x^{k-2 p} \frac{o\left(\tilde{x}^{k-p}\right)}{x^{k-p}}
\end{aligned}
$$

De onde decorre (iii), para qualquer escolha de vetores $\left(\tilde{y}_{0}, A\right):=\left(\tilde{y}_{-}(0), A_{0}, \ldots, A_{2 p-1}\right)$.

Observação 5.1.2 Neste lema

- As expressões $\frac{o\left(\bar{x}^{k-p}\right)}{x^{k-p}}$ dadas acima denotam funções limitadas numa vizinhança do $\tilde{x}= \pm \infty$.

- Na compatificação $X= \pm \frac{1}{\tilde{x}}, Y(X)=X^{p} \tilde{y}_{ \pm}\left( \pm \frac{1}{X}\right)$, o resultado acima diz que $Y(X)=o(1)$, numa vizinhança do $X=0$. 


\subsubsection{Caso $\mathcal{B}_{0}<0$}

Neste caso as funções $J_{k}(\tilde{x})=\int_{0}^{\tilde{x}} \tau^{k} e^{-\mathcal{B}_{0} \frac{\tau^{p+1}}{p+1}} d \tau$ quando $\tilde{x} \rightarrow \infty$ tem a seguinte expansão assintótica

$$
J_{k}(\tilde{x})=\left(\frac{-1}{\mathcal{B}_{0}}\right) \tilde{x}^{k-p} e^{-\mathcal{B}_{0} \frac{\tilde{x}^{p+1}}{p+1}}+o\left(\tilde{x}^{k-p} e^{-\mathcal{B}_{0} \frac{\hat{x}^{p+1}}{p+1}}\right) .
$$

Por outro lado, se $p+1$ é par obtemos

$$
\begin{aligned}
J_{k}(-\tilde{x}) & =(-1)^{k+1} J_{k}(\tilde{x}) \\
& =(-1)^{k+1}\left[\left(\frac{-1}{\mathcal{B}_{0}}\right) \tilde{x}^{k-p} e^{-\mathcal{B}_{0} \frac{\tilde{x}^{p+1}}{p+1}}+o\left(\tilde{x}^{k-p} e^{-\mathcal{B}_{0} \frac{\bar{x}^{p+1}}{p+1}}\right)\right] .
\end{aligned}
$$

E se $p+1$ fosse ímpar

$$
\begin{aligned}
J_{k}(-\tilde{x})= & (-1)^{k+1} \int_{0}^{\tilde{x}} \tau^{k} e^{\mathcal{B}_{0} \frac{\tau^{p+1}}{p+1}} \\
= & (-1)^{k+1}\left[c_{k}\left(-\mathcal{B}_{0}\right)+\left(\frac{-1}{\left|\mathcal{B}_{0}\right|}\right) \tilde{x}^{k-p} e^{\mathcal{B}_{0} \frac{\tilde{x}^{p+1}}{p+1}}+\right. \\
& \left.+o\left(\tilde{x}^{k-p} e^{\mathcal{B}_{0} \frac{\tilde{x}^{p+1}}{p+1}}\right)\right] .
\end{aligned}
$$

Logo daqui, temos o seguinte resultado

Lema 5.1.3 As soluções $\tilde{y}_{ \pm}(\tilde{x})$ numa vizinhança de $\tilde{x}= \pm \infty$ do problema de Cauchy $\left[R_{A, 0}(0), \tilde{y}_{0}\right]$, satisfazem o seguinte

(i) $\tilde{y}_{+}(\tilde{x})=o\left(\tilde{x}^{p}\right)$, numa vizinhança de $\tilde{x}=+\infty$, para qualquer vetor $\left(\tilde{y}_{0}, A\right):=\left(\tilde{y}_{+}(0), A_{0}, \ldots, A_{2 p-1}\right)$,

(ii) Se $p+1$ é par, $\tilde{y}_{-}(\tilde{x})=o\left(\tilde{x}^{p}\right)$ numa vizinhança de $\tilde{x}=-\infty$, para qualquer vetor $\left(\tilde{y}_{0}, A\right):=\left(\tilde{y}_{-}(0), A_{0}, \ldots, A_{2 p-1}\right)$,

(iii) Se $p+1$ é impar, $\tilde{y}_{-}(\tilde{x})=o\left(\tilde{x}^{p}\right)$ numa vizinhança de $\tilde{x}=-\infty$, se e só mente se

$$
\tilde{y}_{0}+\sum_{k=0}^{2 p-1}(-1)^{k+1} c_{k}\left(-\mathcal{B}_{0}\right) A_{k}=0
$$

onde $\tilde{y}_{0}:=\tilde{y}_{-}(0)$.

Demonstração 
(i) Substituindo (5.3) em (5.1), obtemos a igualdade

$$
\frac{\tilde{y}_{+}(\tilde{x})}{\tilde{x}^{p}}=\frac{1}{\tilde{x}^{p}} e^{\mathcal{B}_{0} \frac{\tilde{x}^{p+1}}{p+1}} \tilde{y}_{0}+\left(\frac{-1}{\mathcal{B}_{0}}\right) \sum_{k=0}^{2 p-1} A_{k} \tilde{x}^{k-2 p}+\sum_{k=0}^{2 p-1} x^{k-2 p} \frac{o\left(\tilde{x}^{k-p}\right)}{x^{k-p}} .
$$

Daqui, para escolha arbitrária dos elementos $\left(\tilde{y}_{0}, A\right):=\left(\tilde{y}_{+}(0), A_{0}, \ldots, A_{2 p-1}\right)$, (i) é satisfeito.

(ii) Se $p+1$ é par, definamos $\tilde{y}_{-}(\tilde{x}):=\tilde{y}(-\tilde{x})$ e substituindo (5.4) em (5.1), obtemos analogamente

$$
\begin{aligned}
\frac{\tilde{y}_{-}(\tilde{x})}{\tilde{x}^{p}}= & \frac{1}{\tilde{x}^{p}} e^{\mathcal{B}_{0} \frac{\tilde{x}^{p+1}}{p+1}} \tilde{y}_{0}+\left(\frac{-1}{\mathcal{B}_{0}}\right) \sum_{k=0}^{2 p-1}(-1)^{k+1} A_{k} \tilde{x}^{k-2 p}+ \\
& +\sum_{k=0}^{2 p-1} x^{k-2 p} \frac{o\left(\tilde{x}^{k-p}\right)}{x^{k-p}}
\end{aligned}
$$

satisfazendo (ii), para escolhas arbitrarias de pares $\left(\tilde{y}_{0}, A\right):=\left(\tilde{y}_{+}(0), A_{0}, \ldots, A_{2 p-1}\right)$.

(iii) Se $p+1$ fosse ímpar, definimos $\tilde{y}_{-}(\tilde{x}):=\tilde{y}(-\tilde{x})$ e substituindo (5.5) em (5.1), obtemos a relação

$$
\begin{aligned}
\frac{\tilde{y}_{-}(\tilde{x})}{\tilde{x}^{p}}= & \frac{1}{\tilde{x}^{p}} e^{-\mathcal{B}_{0} \frac{\tilde{x}^{p+1}}{p+1}}\left[\tilde{y}_{0}+\sum_{k=0}^{2 p-1}(-1)^{k+1} c_{k}\left(-\mathcal{B}_{0}\right) A_{k}\right]+ \\
& +\left(\frac{1}{\mathcal{B}_{0}}\right) \sum_{k=0}^{2 p-1}(-1)^{k+1} A_{k} \tilde{x}^{k-2 p}+\sum_{k=0}^{2 p-1} x^{k-2 p} \frac{o\left(\tilde{x}^{k-p}\right)}{x^{k-p}}
\end{aligned}
$$

Assim, (iii) é satisfeito; onde $\tilde{y}_{0}:=\tilde{y}_{-}(0)$.

Observação 5.1.4 Similarmente neste lema

- As expressões $\frac{o\left(\bar{x}^{k-p}\right)}{x^{k-p}}$, dadas acima denotam funções limitadas numa vizinhança do $\tilde{x}= \pm \infty$,

- Nas coordenadas $X= \pm \frac{1}{\tilde{x}}, Y(X)=X^{p} \tilde{y}_{ \pm}\left( \pm \frac{1}{X}\right)$, o resultado acima diz que $Y(X)=o(1)$, numa vizinhança do $X=0$. 


\subsection{O caso $R_{A, B}(0)$}

Neste caso consideremos a família linear de equações diferenciais de primeira ordem

$$
\frac{d \tilde{y}}{d \tilde{x}}=\left(\sum_{k=0}^{2 p-1} A_{k} \tilde{x}^{k}\right)+\left(\sum_{j=0}^{p-1} B_{j} \tilde{x}^{j}+\mathcal{B}_{0} \tilde{x}^{p}\right) \tilde{y}
$$

onde $(A, B)=\left(A_{0}, \ldots, A_{2 p-1}, B_{0}, \ldots, B_{p-1}\right), \quad \mathcal{B}_{0} \neq 0$.

Pela fórmula de variação das constantes, o problema de cauchy associado com condição inicial $\tilde{y}_{0}$, tem soluções únicas dadas por

$$
\tilde{y}(\tilde{x})=e^{P(\tilde{x})}\left[\tilde{y}_{0}+\sum_{k=0}^{2 p-1} A_{k} J_{k}^{P}(\tilde{x})\right],
$$

onde

$$
\begin{aligned}
J_{k}^{P}(\tilde{x}) & =\int_{0}^{\tilde{x}} \tau^{k} e^{-P(\tau)} d \tau, \quad k=0,1, \ldots, 2 p-1 \\
P(\tilde{x}) & =\int_{0}^{\tilde{x}}\left(\sum_{j=0}^{p-1} B_{j} \tau^{j}+\mathcal{B}_{0} \tau^{p}\right) d \tau \\
& =B_{0} \tilde{x}+B_{1} \frac{\tilde{x}^{2}}{2}+\ldots+B_{p-1} \frac{\tilde{x}^{p}}{p}+\mathcal{B}_{0} \frac{\tilde{x}^{p+1}}{p+1}
\end{aligned}
$$

Observemos que

$$
\operatorname{deg}\left(\mathcal{B}_{0} \frac{\tilde{x}^{p+1}}{p+1}\right)=\operatorname{deg}(P(\tilde{x})), \quad P(\tilde{x})=\mathcal{B}_{0} \frac{\tilde{x}^{p+1}}{p+1}(o(1)+1)
$$

Como no caso anterior, o comportamento assintótico destas soluções numa vizinhança de $\tilde{x}= \pm \infty$, dependerá das expansões assintóticas das integrais $J_{k}^{P}(\tilde{x})$ quando $\tilde{x} \rightarrow \pm \infty$; por tanto das constantes $\mathcal{B}_{0}$, e $p+1$.

$\mathrm{O}$ estudo é feito em dois casos.

\subsubsection{Caso $\mathcal{B}_{0}>0$}

Neste caso as funções $J_{k}^{P}(\tilde{x})=\int_{0}^{\bar{x}} \tau^{k} e^{-P(\tau)} d \tau$, são convergentes numa vizinhança de $\tilde{x}=\infty$; logo vale a igualdade

$$
J_{k}^{P}(\tilde{x})=\int_{0}^{\infty} \tau^{k} e^{-P(\tau)} d \tau-\int_{\bar{x}}^{\infty} \tau^{k} e^{-P(\tau)} d \tau .
$$


Como o segundo somando satisfaz

$$
\int_{\bar{x}}^{\infty} \tau^{k} e^{-P(\tau)} d \tau \sim \frac{\tilde{x}^{k} e^{-P(\tilde{x})}}{\left|-P^{\prime}(\tilde{x})\right|}=\frac{\tilde{x}^{k} e^{-P(\tilde{x})}}{\mathcal{B}_{0} \tilde{x}^{p}(o(1)+1)} \sim \frac{\tilde{x}^{k-p} e^{-P(\tilde{x})}}{\mathcal{B}_{0}}
$$

então temos a seguinte igualdade

$$
J_{k}^{P}(\tilde{x})=C_{k}(B)+\left(\frac{-1}{\mathcal{B}_{0}}\right) \tilde{x}^{k-p} e^{-P(\tilde{x})}+o\left(\tilde{x}^{k-p} e^{-P(\tilde{x})}\right),
$$

onde, $C_{k}(B):=\int_{0}^{\infty} \tau^{k} e^{-P(\tau)} d \tau$ é uma função de classe $C^{\infty}$ no parâmetro $B=\left(B_{0}, \ldots, B_{p-1}\right)$ e é tal que $C_{k}(0)=c_{k}\left(\mathcal{B}_{0}\right)$.

Lema 5.2.1 As soluções $\tilde{y}_{ \pm}(\tilde{x})$ numa vizinhança de $\tilde{x}= \pm \infty$ do problema de Cauchy $\left[R_{A, B}(0), \tilde{y}_{0}\right]$, satisfazem o seguinte.

(i) $\tilde{y}_{+}(\tilde{x})=o\left(\tilde{x}^{p}\right)$, numa vizinhança de $\tilde{x}=+\infty$ se e só se

$$
\tilde{y}_{0}+\sum_{k=0}^{2 p-1} C_{k}(B) A_{k}=0
$$

onde $\tilde{y}_{0}:=\tilde{y}_{+}(0), B=\left(B_{0}, \ldots, B_{p-1}\right)$.

(ii) Se $p+1$ é par, $\tilde{y}_{-}(\tilde{x})=o\left(\tilde{x}^{p}\right)$ numa vizinhança de $\tilde{x}=-\infty$, se e só se

$$
\tilde{y}_{0}+\sum_{k=0}^{2 p-1}(-1)^{k+1} C_{k}(\bar{B}) A_{k}=0,
$$

onde $\tilde{y}_{0}:=\tilde{y}_{-}(0), \bar{B}=\left(-B_{0}, \ldots,(-1)^{k+1} B_{k}, \ldots,-B_{p-1}\right)$.

(iii) Se $p+1$ é ímpar, $\tilde{y}_{-}(\tilde{x})=o\left(\tilde{x}^{p}\right)$ numa vizinhança de $\tilde{x}=-\infty$, para qualquer vetor $\left(\tilde{y}_{0}, A, B\right):=\left(\tilde{y}_{-}(0), A_{0}, \ldots, A_{2 p-1}, B_{0}, \ldots, B_{p-1}\right)$.

DEMONSTRAÇÃO

(i) Substituindo (5.7) em (5.6), obtemos a solução $\tilde{y}_{+}(\tilde{x})$ dada por

$$
\tilde{y}_{+}(\tilde{x})=e^{P(\bar{x})}\left[\tilde{y}_{0}+\sum_{k=0}^{2 p-1} C_{k}(B) A_{k}\right]+\left(\frac{-1}{\mathcal{B}_{0}}\right) \sum_{k=0}^{2 p-1} A_{k} \tilde{x}^{k-p}+\sum_{k=0}^{2 p-1} o\left(\tilde{x}^{k-p}\right) .
$$

Daqui, resulta que

$$
\begin{aligned}
\frac{\tilde{y}_{+}(\tilde{x})}{\tilde{x}^{p}}= & \frac{1}{\tilde{x}^{p}} e^{P(\tilde{x})}\left[\tilde{y}_{0}+\sum_{k=0}^{2 p-1} C_{k}(B) A_{k}\right]+\left(\frac{-1}{\mathcal{B}_{0}}\right) \sum_{k=0}^{2 p-1} A_{k} \tilde{x}^{k-2 p}+ \\
& +\sum_{k=0}^{2 p-1} x^{k-2 p} \frac{O\left(\tilde{x}^{k-p}\right)}{x^{k-p}},
\end{aligned}
$$

onde, $\tilde{y}_{0}:=\tilde{y}_{+}(0)$; $\log$ (i) é satisfeito, para arbitrários $(A, B) \in \mathbb{R}^{3 p}$. 
(ii) Se $p+1$ é par, vale

$$
-P(-\tilde{x})=-\sum_{j=0}^{p-1}(-1)^{j+1} B_{j} \frac{\tilde{x}^{j+1}}{j+1}-\mathcal{B}_{0} \frac{\tilde{x}^{p+1}}{p+1} .
$$

Logo obtemos

$$
\begin{aligned}
J_{k}^{P}(-\tilde{x})= & (-1)^{k+1}\left[C_{k}(\bar{B})+\left(\frac{-1}{\mathcal{B}_{0}}\right) \tilde{x}^{k-p} e^{-P(-\bar{x})}+\right. \\
& \left.+o\left(\tilde{x}^{k-p} e^{-P(-\bar{x})}\right)\right] .
\end{aligned}
$$

Onde $\bar{B}=\left(-B_{0}, \ldots,(-1)^{k+1} B_{k}, \ldots,-B_{p-1}\right)$.

Assim definindo $\tilde{y}_{-}(\tilde{x}):=\tilde{y}(-\tilde{x})$ e usando (5.6) e (5.8) resulta que

$$
\begin{aligned}
\frac{\tilde{y}_{-}(\tilde{x})}{\tilde{x}^{p}}= & \frac{1}{\tilde{x}^{p}} e^{P(-\tilde{x})}\left[\tilde{y}_{0}+\sum_{k=0}^{2 p-1}(-1)^{k+1} C_{k}(\bar{B}) A_{k}\right]+ \\
& +\left(\frac{-1}{\mathcal{B}_{0}}\right) \sum_{k=0}^{2 p-1}(-1)^{k+1} A_{k} \tilde{x}^{k-2 p}+\sum_{k=0}^{2 p-1} x^{k-2 p} \frac{o\left(\tilde{x}^{k-p}\right)}{x^{k-p}} \\
= & \frac{1}{\tilde{x}^{p}} e^{P(-\tilde{x})}\left[\tilde{y}_{0}+\sum_{k=0}^{2 p-1}(-1)^{k+1} C_{k}(\bar{B}) A_{k}\right]+ \\
& +\sum_{k=0}^{2 p-1}\left[\frac{1}{\mathcal{B}_{0}}(-1)^{k} A_{k}+O(1)\right] \tilde{x}^{k-2 p}
\end{aligned}
$$

onde $\tilde{y}_{0}:=\tilde{y}_{-}(0)$; logo (ii) é satisfeito para parâmetros arbitrários $(A, B) \in \mathbb{R}^{3 p}$.

(iii) Se $p+1$ é ímpar vale

$$
-P(-\tilde{x})=-\sum_{j=0}^{p-1}(-1)^{j+1} B_{j} \frac{\tilde{x}^{j+1}}{j+1}+\mathcal{B}_{0} \frac{\tilde{x}^{p+1}}{p+1} .
$$

Logo obtemos

$$
\begin{aligned}
J_{k}^{P}(-\tilde{x})= & (-1)^{k+1}\left[\left(\frac{1}{\mathcal{B}_{0}}\right) \tilde{x}^{k-p} e^{-P(-\bar{x})}+\right. \\
& \left.+o\left(\bar{x}^{k-p} e^{-P(-\bar{x})}\right)\right] .
\end{aligned}
$$


Definindo $\tilde{y}_{-}(\tilde{x}):=\tilde{y}(-\tilde{x})$ e usando $(5.6)$ e (5.9) resulta que

$$
\begin{aligned}
\frac{\tilde{y}_{-}(\tilde{x})}{\tilde{x}^{p}}= & \frac{1}{\tilde{x}^{p}} e^{P(-\tilde{x})} \tilde{y}_{0}+\left(\frac{1}{\mathcal{B}_{0}}\right) \sum_{k=0}^{2 p-1}(-1)^{k+1} A_{k} \tilde{x}^{k-2 p}+ \\
& +\sum_{k=0}^{2 p-1} x^{k-2 p} \frac{o\left(\tilde{x}^{k-p}\right)}{x^{k-p}} \\
= & \frac{1}{\tilde{x}^{p}} e^{P(-\tilde{x})} \tilde{y}_{0}+\sum_{k=0}^{2 p-1}\left[\frac{1}{\mathcal{B}_{0}}(-1)^{k+1} A_{k}+O(1)\right] \tilde{x}^{k-2 p}
\end{aligned}
$$

onde $\tilde{y}_{0}:=\tilde{y}_{-}(0)$; logo (iii) é satisfeito para arbitrária escolha de vetores $\left(\tilde{y}_{0}, A, B\right) \in \mathbb{R}^{3 p+1}$.

\subsubsection{Caso $\mathcal{B}_{0}<0$}

Neste caso as integrais $J_{k}^{P}(\tilde{x})=\int_{0}^{\tilde{x}} \tau^{k} e^{-P(\tau)} d \tau$, numa vizinhança de $\tilde{x}=+\infty$ possuem a seguinte expansão assintótica

$$
J_{k}^{P}(\tilde{x})=\left(\frac{-1}{\mathcal{B}_{0}}\right) \tilde{x}^{k-p} e^{-P(\tilde{x})}+o\left(\tilde{x}^{k-p} e^{-P(\bar{x})}\right) .
$$

Lema 5.2.2 As soluções $\tilde{y}_{ \pm}(\tilde{x})$ numa vizinhança de $\tilde{x}= \pm \infty$ do problema de Cauchy $\left[R_{A, B}(0), \tilde{y}_{0}\right]$, satisfazem o seguinte

(i) $\tilde{y}_{+}(\tilde{x})=o\left(\tilde{x}^{p}\right)$, numa vizinhança de $\tilde{x}=+\infty$, para qualquer vetor $\left(\tilde{y}_{0}, A, B\right):=\left(\tilde{y}_{+}(0), A_{0}, \ldots, A_{2 p-1}, B_{0}, \ldots, B_{p-1}\right)$.

(ii) Se $p+1$ é par, $\tilde{y}_{-}(\tilde{x})=o\left(\tilde{x}^{p}\right)$ numa vizinhança de $\tilde{x}=-\infty$, para qualquer vetor $\left(\tilde{y}_{0}, A, B\right):=\left(\tilde{y}_{-}(0), A_{0}, \ldots, A_{2 p-1}, B_{0}, \ldots, B_{p-1}\right)$.

(iii) Se $p+1$ é impar, $\tilde{y}_{-}(\tilde{x})=o\left(\tilde{x}^{p}\right)$ numa vizinhança de $\tilde{x}=-\infty$, se e só se

$$
\tilde{y}_{0}+\sum_{k=0}^{2 p-1}(-1)^{k+1} C_{k}(\tilde{B}) A_{k}=0,
$$

onde $\tilde{y}_{0}:=\tilde{y}_{-}(0), \tilde{B}=\left(-B_{0}, \ldots(-1)^{k+1} B_{k}, \ldots, B_{p-1}\right)$. 


\section{DEMONSTRAÇÃo}

(i) Usando (5.6) e (5.10) resulta que

$$
\begin{aligned}
\frac{\tilde{y}_{+}(\tilde{x})}{\tilde{x}^{p}} & =\frac{1}{\tilde{x}^{p}} e^{P(\tilde{x})} \tilde{y}_{0}+\left(\frac{-1}{\mathcal{B}_{0}}\right) \sum_{k=0}^{2 p-1} A_{k} \tilde{x}^{k-2 p}+\sum_{k=0}^{2 p-1} x^{k-2 p} \frac{o\left(\tilde{x}^{k-p}\right)}{x^{k-p}} \\
& =\frac{1}{\tilde{x}^{p}} e^{P(\tilde{x})} \tilde{y}_{0}+\sum_{k=0}^{2 p-1}\left[\left(\frac{-1}{\mathcal{B}_{0}}\right) A_{k}+O(1)\right] \tilde{x}^{k-2 p},
\end{aligned}
$$

onde $\tilde{y}_{0}:=\tilde{y}_{+}(0)$, e assim (i) é satisfeito para qualquer escolha de vetores $\left(\tilde{y}_{0}, A, B\right) \in \mathbb{R}^{3 p+1}$.

(ii) Se $p+1$ é par, vale

$$
-P(-\tilde{x})=-\sum_{j=0}^{p-1}(-1)^{j+1} B_{j} \frac{\tilde{x}^{j+1}}{j+1}-\mathcal{B}_{0} \frac{\tilde{x}^{p+1}}{p+1} .
$$

Logo obtemos

$$
\begin{aligned}
J_{k}^{P}(-\tilde{x})= & (-1)^{k+1}\left[\left(\frac{-1}{\mathcal{B}_{0}}\right) \tilde{x}^{k-p} e^{-P(-\tilde{x})}+\right. \\
& \left.+o\left(\tilde{x}^{k-p} e^{-P(-\tilde{x})}\right)\right] .
\end{aligned}
$$

Daqui definindo $\tilde{y}_{-}(\tilde{x}):=\tilde{y}(-\tilde{x})$ e usando (5.6) e (5.11) resulta que

$$
\begin{aligned}
\frac{\tilde{y}_{-}(\tilde{x})}{\tilde{x}^{p}}= & \frac{1}{\tilde{x}^{p}} e^{P(-\tilde{x})} \tilde{y}_{0}+\left(\frac{-1}{\mathcal{B}_{0}}\right) \sum_{k=0}^{2 p-1}(-1)^{k+1} A_{k} \tilde{x}^{k-2 p}+ \\
& +\sum_{k=0}^{2 p-1} x^{k-2 p} \frac{o\left(\tilde{x}^{k-p}\right)}{x^{k-p}} \\
= & \frac{1}{\tilde{x}^{p}} e^{P(-\bar{x})} \tilde{y}_{0}+\sum_{k=0}^{2 p-1}\left[\frac{1}{\mathcal{B}_{0}}(-1)^{k} A_{k}+O(1)\right] \tilde{x}^{k-2 p} .
\end{aligned}
$$

onde $\tilde{y}_{0}:=\tilde{y}_{-}(0)$; logo (ii) é satisfeito para arbitrária escolha de vetores $\left(\tilde{y}_{0}, A, B\right) \in \mathbb{R}^{3 p+1}$.

(iii) Se $p+1$ fosse ímpar, então vale

$$
-P(-\tilde{x})=-\sum_{j=0}^{p-1}(-1)^{j+1} B_{j} \frac{\tilde{x}^{j+1}}{j+1}+\mathcal{B}_{0} \frac{\tilde{x}^{p+1}}{p+1} .
$$


Logo as integrais $J_{k}^{P}(-\tilde{x})=\int_{0}^{-\bar{x}} \tau^{k} e^{-P(\tau)} d \tau$ são convergentes, e quando $\tilde{x} \rightarrow+\infty$, podemos escrever a seguinte expansão assintótica

$$
\begin{aligned}
J_{k}^{P}(-\tilde{x})= & (-1)^{k+1}\left[C_{k}(\tilde{B})+\left(\frac{-1}{\left|\mathcal{B}_{0}\right|}\right) \tilde{x}^{k-p} e^{-P(-\tilde{x})}+\right. \\
& \left.+o\left(\tilde{x}^{k-p} e^{-P(-\tilde{x})}\right)\right],
\end{aligned}
$$

onde $\tilde{B}=\left(-B_{0}, \ldots,(-1)^{k+1} B_{k}, \ldots, B_{p-1}\right)$.

Daqui definindo $\tilde{y}_{-}(\tilde{x}):=\tilde{y}(-\tilde{x})$ e usando (5.6) e (5.12) resulta que

$$
\begin{aligned}
\frac{\tilde{y}-(\tilde{x})}{\tilde{x}^{p}}= & \frac{1}{\tilde{x}^{p}} e^{P(-\tilde{x})}\left[\tilde{y}_{0}+\sum_{k=0}^{2 p-1}(-1)^{k+1} C_{k}(\tilde{B}) A_{k}\right]+ \\
& +\left(\frac{1}{\mathcal{B}_{0}}\right) \sum_{k=0}^{2 p-1}(-1)^{k+1} A_{k} \tilde{x}^{k-2 p}+\sum_{k=0}^{2 p-1} x^{k-2 p} \frac{o\left(\tilde{x}^{k-p}\right)}{x^{k-p}} \\
= & \frac{1}{\tilde{x}^{p}} e^{P(-\bar{x})}\left[\tilde{y}_{0}+\sum_{k=0}^{2 p-1}(-1)^{k+1} C_{k}(\tilde{B}) A_{k}\right]+ \\
& +\sum_{k=0}^{2 p-1}\left[\frac{1}{\mathcal{B}_{0}}(-1)^{k+1} A_{k}+O(1)\right] \tilde{x}^{k-2 p}
\end{aligned}
$$

onde $\tilde{y}_{0}:=\tilde{y}_{-}(0)$; logo (iii) é satisfeito para arbitraria escolha de parâmetros $(A, B) \in \mathbb{R}^{3 p}$.

Observação 5.2.3 Das seções anteriores, notemos que

(1) $S e \mathcal{B}_{0}>0$, e p +1 par; $C_{k}(B=0)=C_{k}(\bar{B}=0)=c_{k}\left(\mathcal{B}_{0}\right)$;

(2) Se $\mathcal{B}_{0}<0$, e $p+1$ impar; $C_{k}(\tilde{B}=0)=c_{k}\left(-\mathcal{B}_{0}\right)$;

(3) Para os respectivos casos onde aparecem as funções

$$
C_{k}(B), C_{k}(\bar{B}), c_{k}\left(\mathcal{B}_{0}\right), \quad \text { e } \quad C_{k}(\tilde{B}), c_{k}\left(-\mathcal{B}_{0}\right) ;
$$

as condições iniciais $\tilde{y}_{0}:=\tilde{y}_{ \pm}(0)$ são funções lineares dos parâmetros $(A, B)$. 
Por exemplo no caso $\left[\mathcal{B}_{0}>0, p+1\right.$ par $]$ do lema 5.2.1 temos que a função $\tilde{y}_{+}(0)-\tilde{y}_{-}(0)$ é dada por

$$
\tilde{y}_{+}(0)-\tilde{y}_{-}(0)=-\sum_{k=0}^{2 p-1}\left[C_{k}(B)+(-1)^{k} C_{k}(\bar{B})\right] A_{k},
$$

para cada $(A, B)$ no domínio comum de definição contido em $U \in$ $\left(\mathbb{R}^{3 p}, 0\right)$.

\subsection{O caso $R_{0,0}\left(\mathcal{Q}_{0}\right)$}

Neste caso vamos considerar a equação diferencial não linear dada por

$$
\frac{d \tilde{y}}{d \tilde{x}}=\mathcal{B}_{0} \tilde{x}^{p} \tilde{y}+\mathcal{Q}_{0} \tilde{y}^{2}
$$

a qual corresponde à família Riccati $R_{A, B}\left(\mathcal{Q}_{0}\right)$, situada na origem $(A, B)=$ $(0,0)$ do espaço de parâmetros $\mathbb{R}^{3 p}$.

É claro que uma solução é dada pela função nula $\tilde{y}(\tilde{x})=0$, outra solução não trivial para o problema de Cauchy $\left[R_{0,0}\left(\mathcal{Q}_{0}\right), \tilde{y}_{0}\right]$, com $\tilde{y}_{0}=\tilde{y}\left(\tilde{x}_{0}\right) \neq 0$, é espresa explicitamente por

$$
\tilde{y}(\tilde{x})=\frac{e^{\mathcal{B}_{0} \frac{\bar{x}^{p+1}}{p+1}}}{\frac{e^{\mathcal{B}_{0}} \frac{\tilde{x}_{0}^{p+1}}{p+1}}{\tilde{y}_{0}}-\mathcal{Q}_{0} \int_{\tilde{x}_{0}}^{\tilde{x}} e^{\mathcal{B}_{0} \frac{t^{p+1}}{p+1}} d t},
$$

onde $\tilde{x}_{0} \in(-\infty,+\infty)$, (ver Apêndice 1 ).

Esta solução, está bem definida se o denominador é diferente de zero. Nas seções a seguir, vamos fazer um estudo cuidadoso da função

$$
L\left(\tilde{x}, \tilde{x}_{0}\right)=\mathcal{Q}_{0} \int_{\tilde{x}_{0}}^{\tilde{x}} e^{\mathcal{B}_{0} \frac{t^{p+1}}{p+1}} d t .
$$

A seguir, vamos usar este estudo para estimar o intervalo maximal de existência de uma solução de $R_{A, B}\left(\mathcal{Q}_{0}\right)$ para $(A, B)$ suficientemente pequenos.

\subsubsection{Caso $\mathrm{p}+1$ par}

Tomemos as integrais $c_{0}\left(\mathcal{B}_{0}\right)$ e $c_{0}\left(-\mathcal{B}_{0}\right)$ definidas nas seções anteriores, que podem ser escritas como

$$
c_{0}\left(\left|\mathcal{B}_{0}\right|\right)=\int_{0}^{\infty} e^{-\left|\mathcal{B}_{0}\right| \frac{\tau^{p+1}}{p+1}} d \tau=\left|\mathcal{B}_{0}\right|^{\frac{-1}{p+1}}(p+1)^{\frac{1}{p+1}-1} \Gamma\left(\frac{1}{p+1}\right) .
$$


Temos as seguintes estimativas para $L\left(\tilde{x}, \tilde{x}_{0}\right)$.

Lema 5.3.1 Suponha que

(i) $\operatorname{Se}\left[\mathcal{Q}_{0}>0, \mathcal{B}_{0}<0, \tilde{x}_{0} \leq 0\right]$, ou $\left[\mathcal{Q}_{0}<0, \mathcal{B}_{0}<0, \tilde{x}_{0} \geq 0\right]$, então

$$
-\lambda e^{\mathcal{B}_{0} \frac{\left|\tilde{x}_{0}\right|^{p+1}}{p+1}}<L\left(\tilde{x}, \tilde{x}_{0}\right)<2 \lambda
$$

(ii) Se $\left[\mathcal{Q}_{0}<0, \mathcal{B}_{0}<0, \tilde{x}_{0} \leq 0\right]$, ou $\left[\mathcal{Q}_{0}>0, \mathcal{B}_{0}<0, \tilde{x}_{0} \geq 0\right]$, então

$$
-2 \lambda<L\left(\tilde{x}, \tilde{x}_{0}\right)<\lambda e^{\mathcal{B}_{0} \frac{\left|\tilde{x}_{0}\right|^{p+1}}{p+1}}
$$

onde $\lambda:=\left|\mathcal{Q}_{0}\right| c_{0}\left(\left|\mathcal{B}_{0}\right|\right)$.

\section{Demonstração}

(i) - Para $\left[\mathcal{Q}_{0}>0, \mathcal{B}_{0}<0, \tilde{x}_{0} \leq 0\right]$, temos as seguintes desigualdades: Se $-\infty<x<x_{0}$, com a mudança de variável $x_{0}-t=u$, obtemos

$$
\begin{aligned}
L\left(\tilde{x}, \tilde{x}_{0}\right) & =-\mathcal{Q}_{0} \int_{0}^{\tilde{x}_{0}-\bar{x}} e^{\mathcal{B}_{0} \frac{\left(u-\tilde{x}_{0} p^{p+1}\right.}{p+1}} d u \\
& >-\mathcal{Q}_{0}\left(\int_{0}^{\tilde{x}_{0}-\bar{x}} e^{\mathcal{B}_{0} \frac{u^{p+1}}{p+1}} d u\right) e^{\mathcal{B}_{0} \frac{\mid \tilde{x}_{0} p^{p+1}}{p+1}} \\
& \geq-\left|\mathcal{Q}_{0}\right|\left(\int_{0}^{\infty} e^{\mathcal{B}_{0} \frac{u^{p+1}}{p+1}} d u\right) e^{\mathcal{B}_{0} \frac{\left|\underline{x}_{0}\right|^{p+1}}{p+1}} \\
& =-\left|\mathcal{Q}_{0}\right| c_{0}\left(\left|\mathcal{B}_{0}\right|\right) e^{\mathcal{B}_{0} \frac{\left|\tilde{x}_{0}\right|^{p+1}}{p+1}}
\end{aligned}
$$

Por outro lado, se $\tilde{x}_{0}<\tilde{x}$ obtemos

$$
\begin{aligned}
L\left(\tilde{x}, \tilde{x}_{0}\right) & =\mathcal{Q}_{0} \int_{\tilde{x}_{0}}^{\tilde{x}} e^{\mathcal{B}_{0} \frac{t^{p+1}}{p+1}} d t<2 \mathcal{Q}_{0} \int_{0}^{\infty} e^{\mathcal{B}_{0} \frac{t^{p+1}}{p+1}} d t \\
& =2\left|\mathcal{Q}_{0}\right| c_{0}\left(\left|\mathcal{B}_{0}\right|\right) .
\end{aligned}
$$

Aqui usamos a desigualdade

$$
\left(u-\tilde{x}_{0}\right)^{p+1}=\left(u+\left|\tilde{x}_{0}\right|\right)^{p+1} \geq u^{p+1}+\left|\tilde{x}_{0}\right|^{p+1} .
$$

De forma análoga 
- Para $\left[\mathcal{Q}_{0}<0, \mathcal{B}_{0}<0, \tilde{x}_{0} \geq 0\right]$, temos as seguintes desigualdades: Se $-\infty<\tilde{x}<\tilde{x}_{0}$

$$
\begin{aligned}
L\left(\tilde{x}, \tilde{x}_{0}\right) & =-\mathcal{Q}_{0} \int_{\tilde{x}}^{\tilde{x}_{0}} e^{\mathcal{B}_{0} \frac{t^{p+1}}{p+1}} d t<-2 \mathcal{Q}_{0} \int_{0}^{\infty} e^{\mathcal{B}_{0} \frac{t^{p+1}}{p+1}} d t \\
& =2\left|\mathcal{Q}_{0}\right| c_{0}\left(\left|\mathcal{B}_{0}\right|\right) .
\end{aligned}
$$

Por outro lado, se $\tilde{x}_{0}<\tilde{x}$, com a mudança de variável $t-\tilde{x}_{0}=u$ obtemos

$$
\begin{aligned}
L\left(\tilde{x}, \tilde{x}_{0}\right) & =\mathcal{Q}_{0} \int_{0}^{\tilde{x}-\tilde{x}_{0}} e^{\mathcal{B}_{0} \frac{\left(u+\tilde{x}_{0}\right)^{p+1}}{p+1}} d u \\
& >\mathcal{Q}_{0}\left(\int_{0}^{\tilde{x}-\tilde{x}_{0}} e^{\mathcal{B}_{0} \frac{u^{p+1}}{p+1}} d u\right) e^{\mathcal{B}_{0} \frac{\left|\tilde{x}_{0}\right|^{p+1}}{p+1}} \\
& \geq \mathcal{Q}_{0}\left(\int_{0}^{\infty} e^{\mathcal{B}_{0} \frac{u^{p+1}}{p+1}} d u\right) e^{\mathcal{B}_{0} \frac{\left|\tilde{x}_{0}\right|^{p+1}}{p+1}} \\
& =-\left|\mathcal{Q}_{0}\right| c_{0}\left(\left|\mathcal{B}_{0}\right|\right) e^{\mathcal{B}_{0} \frac{\left|\tilde{x}_{0}\right|^{p+1}}{p+1}} .
\end{aligned}
$$

Aqui usamos a desigualdade

$$
\left(u+\tilde{x}_{0}\right)^{p+1} \geq u^{p+1}+\left|\tilde{x}_{0}\right|^{p+1} .
$$

(ii) - Para $\left[\mathcal{Q}_{0}<0, \mathcal{B}_{0}<0, \tilde{x}_{0} \leq 0\right]$, temos as seguintes desigualdades: Se $-\infty<\tilde{x}<\tilde{x}_{0}$, com a mudança $\tilde{x}_{0}-t=u$, obtemos

$$
\begin{aligned}
L\left(\tilde{x}, \tilde{x}_{0}\right) & =-\mathcal{Q}_{0} \int_{0}^{\tilde{x}_{0}-\tilde{x}} e^{\mathcal{B}_{0} \frac{\left(u-\tilde{x}_{0}\right)^{p+1}}{p+1}} d u \\
& <-\mathcal{Q}_{0}\left(\int_{0}^{\tilde{x}_{0}-\tilde{x}} e^{\mathcal{B}_{0} \frac{u^{p+1}}{p+1}} d u\right) e^{\mathcal{B}_{0} \frac{\left|\tilde{x}_{0}\right|^{p+1}}{p+1}} \\
& \leq-\mathcal{Q}_{0}\left(\int_{0}^{\infty} e^{\mathcal{B}_{0} \frac{u^{p+1}}{p+1}} d u\right) e^{\mathcal{B}_{0} \frac{\left|\tilde{x}_{0}\right|^{p+1}}{p+1}} \\
& =\left|\mathcal{Q}_{0}\right| c_{0}\left(\left|\mathcal{B}_{0}\right|\right) e^{\mathcal{B}_{0} \frac{\left|\tilde{x}_{0}\right|^{p+1}}{p+1}} .
\end{aligned}
$$

Por outro lado se, $\tilde{x}_{0}<\tilde{x}$

$$
\begin{aligned}
L\left(\tilde{x}, \tilde{x}_{0}\right) & =\mathcal{Q}_{0} \int_{\bar{x}_{0}}^{\bar{x}} e^{\mathcal{B}_{0} \frac{t^{p+1}}{p+1}} d t>2 \mathcal{Q}_{0} \int_{0}^{\infty} e^{\mathcal{B}_{0} \frac{t^{p+1}}{p+1}} d t \\
& =-2\left|\mathcal{Q}_{0}\right| c_{0}\left(\left|\mathcal{B}_{0}\right|\right) .
\end{aligned}
$$

De forma análoga 
- Para $\left[\mathcal{Q}_{0}>0, \mathcal{B}_{0}<0, \tilde{x}_{0} \geq 0\right]$, temos as seguintes desigualdades: Se $-\infty<\tilde{x}<\tilde{x}_{0}$, obtemos

$$
\begin{aligned}
L\left(\tilde{x}, \tilde{x}_{0}\right) & =\mathcal{Q}_{0} \int_{\bar{x}_{0}}^{\tilde{x}} e^{\mathcal{B}_{0} \frac{t^{p+1}}{p+1}} d t>-2 \mathcal{Q}_{0} \int_{0}^{\infty} e^{\mathcal{B}_{0} \frac{t^{p+1}}{p+1}} d t \\
& =-2\left|\mathcal{Q}_{0}\right| c_{0}\left(\left|\mathcal{B}_{0}\right|\right) .
\end{aligned}
$$

Por outro lado se, $\tilde{x}_{0}<\tilde{x}$, com a mudança $t-\tilde{x}_{0}=u$, obtemos

$$
\begin{aligned}
L\left(\tilde{x}, \tilde{x}_{0}\right) & =\mathcal{Q}_{0} \int_{0}^{\tilde{x}-\tilde{x}_{0}} e^{\mathcal{B}_{0} \frac{\left(u+\tilde{x}_{0} p^{p+1}\right.}{p+1}} d u \\
& <\mathcal{Q}_{0}\left(\int_{0}^{\tilde{x}-\tilde{x}_{0}} e^{\mathcal{B}_{0} \frac{u^{p+1}}{p+1}} d u\right) e^{\mathcal{B}_{0} \frac{\left|\tilde{x}_{0}\right|^{p+1}}{p+1}} \\
& \leq \mathcal{Q}_{0}\left(\int_{0}^{\infty} e^{\mathcal{B}_{0} \frac{u^{p+1}}{p+1}} d u\right) e^{\mathcal{B}_{0} \frac{\left|\tilde{x}_{0}\right|^{p+1}}{p+1}} \\
& =\left|\mathcal{Q}_{0}\right| c_{0}\left(\left|\mathcal{B}_{0}\right|\right) e^{\mathcal{B}_{0} \frac{\left|\tilde{x}_{0}\right|^{p+1}}{p+1}} .
\end{aligned}
$$

\subsubsection{Caso $\mathrm{p}+1$ ímpar}

Neste caso, temos as seguintes estimativas.

Lema 5.3.2 Suponha que

(i) $\operatorname{Se}\left[\mathcal{Q}_{0}>0, \mathcal{B}_{0}>0, \tilde{x}_{0} \leq 0\right]$, ou $\left[\mathcal{Q}_{0}<0, \mathcal{B}_{0}<0, \tilde{x}_{0} \geq 0\right]$, então

$$
L\left(\tilde{x}, \tilde{x}_{0}\right) \geq-\lambda e^{\mathcal{B}_{0} \frac{\bar{x}_{0}^{p+1}}{p+1}} .
$$

(ii) $\operatorname{Se}\left[\mathcal{Q}_{0}<0, \mathcal{B}_{0}>0, \tilde{x}_{0} \leq 0\right]$, ou $\left[\mathcal{Q}_{0}>0, \mathcal{B}_{0}<0, \tilde{x}_{0} \geq 0\right]$, então

$$
L\left(\tilde{x}, \tilde{x}_{0}\right) \leq \lambda e^{\mathcal{B}_{0} \frac{\dot{x}_{0}^{p+1}}{p+1}} .
$$

onde $\lambda:=\left|\mathcal{Q}_{0}\right| c_{0}\left(\left|\mathcal{B}_{0}\right|\right)$.

DEMONSTRAÇÃO 
(i) - Para $\left[\mathcal{Q}_{0}>0, \mathcal{B}_{0}>0, \tilde{x}_{0} \leq 0\right]$, temos as seguintes desigualdades: Se $-\infty<\tilde{x}<\tilde{x}_{0}$, com a mudança $\tilde{x}_{0}-t=u$ obtemos

$$
\begin{aligned}
L\left(\tilde{x}, \tilde{x}_{0}\right) & =-\mathcal{Q}_{0} \int_{0}^{\tilde{x}_{0}-\tilde{x}} e^{-\mathcal{B}_{0} \frac{\left(u+\left|\tilde{x}_{0}\right|\right)^{p+1}}{p+1}} d u \\
& \geq-\mathcal{Q}_{0}\left(\int_{0}^{\tilde{x}_{0}-\tilde{x}} e^{-\mathcal{B}_{0} \frac{u^{p+1}}{p+1}} d u\right) e^{-\mathcal{B}_{0} \frac{\left|\bar{x}_{0}\right|^{p+1}}{p+1}} \\
& \geq-\mathcal{Q}_{0}\left(\int_{0}^{\infty} e^{-\mathcal{B}_{0} \frac{u^{p+1}}{p+1}} d u\right) e^{\mathcal{B}_{0} \frac{\tilde{x}_{0}^{p+1}}{p+1}} \\
& =-\left|\mathcal{Q}_{0}\right| c_{0}\left(\left|\mathcal{B}_{0}\right|\right) e^{\mathcal{B}_{0} \frac{\tilde{x}_{0}^{p+1}}{p+1}}
\end{aligned}
$$

\section{Análogamente}

- Para $\left[\mathcal{Q}_{0}<0, \mathcal{B}_{0}<0, \tilde{x}_{0} \geq 0\right]$, temos as seguintes desigualdades: Se $\tilde{x}_{0}<\tilde{x}$, com a mudança $t-\tilde{x}_{0}=u$ obtemos

$$
\begin{aligned}
L\left(\tilde{x}, \tilde{x}_{0}\right) & =\mathcal{Q}_{0} \int_{0}^{\bar{x}-\bar{x}_{0}} e^{\mathcal{B}_{0} \frac{\left(u+\bar{x}_{0}\right)^{p+1}}{p+1}} d u \\
& \geq \mathcal{Q}_{0}\left(\int_{0}^{\tilde{x}-\bar{x}_{0}} e^{\mathcal{B}_{0} \frac{u^{p+1}}{p+1}} d u\right) e^{\mathcal{B}_{0} \frac{\left|\tilde{x}_{0}\right| p^{p+1}}{p+1}} \\
& \geq-\left|\mathcal{Q}_{0}\right|\left(\int_{0}^{\infty} e^{\mathcal{B}_{0} \frac{u^{p+1}}{p+1}} d u\right) e^{\mathcal{B}_{0} \frac{\left|\tilde{x}_{0}\right|^{p+1}}{p+1}} \\
& =-\left|\mathcal{Q}_{0}\right| c_{0}\left(\left|\mathcal{B}_{0}\right|\right) e^{\mathcal{B}_{0} \frac{\left|\tilde{x}_{0}\right|^{p+1}}{p+1}}
\end{aligned}
$$

(ii) - Para $\left[\mathcal{Q}_{0}<0, \mathcal{B}_{0}>0, \tilde{x}_{0} \leq 0\right]$, temos as seguintes desigualdades: Se $\infty<\tilde{x}<\tilde{x}_{0}$, com a mudança $\tilde{x}_{0}-t=u$ obtemos

$$
\begin{aligned}
L\left(\tilde{x}, \tilde{x}_{0}\right) & =-\mathcal{Q}_{0} \int_{0}^{\tilde{x}_{0}-\bar{x}} e^{-\mathcal{B}_{0} \frac{\left(u+\left|\tilde{x}_{0}\right|\right)^{p+1}}{p+1}} d u \\
& \leq-\mathcal{Q}_{0}\left(\int_{0}^{\dot{x}_{0}-\tilde{x}} e^{-\mathcal{B}_{0} \frac{u^{p+1}}{p+1}} d u\right) e^{\mathcal{B}_{0} \frac{\dot{x}_{0}^{p+1}}{p+1}} \\
& \leq\left|\mathcal{Q}_{0}\right|\left(\int_{0}^{\infty} e^{-\mathcal{B}_{0} \frac{u^{p+1}}{p+1}} d u\right) e^{\mathcal{B}_{0} \frac{\dot{x}_{0}^{p+1}}{p+1}} \\
& =\left|\mathcal{Q}_{0}\right| c_{0}\left(\left|\mathcal{B}_{0}\right|\right) e^{\mathcal{B}_{0} \frac{\tilde{x}_{0}^{p+1}}{p+1}} .
\end{aligned}
$$

- Para $\left[\mathcal{Q}_{0}>0, \mathcal{B}_{0}<0, \tilde{x}_{0} \geq 0\right]$, temos as seguintes desigualdades: 
74 CAPÍTULO 5. DINÂMICA ASSINTÓTICA SOBRE A CARTA $K_{\bar{\varepsilon}}$

Se $\tilde{x}_{0}<\tilde{x}$, com a mudança $t-\tilde{x}_{0}=u$ obtemos

$$
\begin{aligned}
L\left(\tilde{x}, \tilde{x}_{0}\right) & =\mathcal{Q}_{0} \int_{0}^{\tilde{x}-\bar{x}_{0}} e^{\mathcal{B}_{0} \frac{\left(u+\bar{x}_{0}\right)^{p+1}}{p+1}} d u \\
& \leq \mathcal{Q}_{0}\left(\int_{0}^{\tilde{x}-\bar{x}_{0}} e^{\mathcal{B}_{0} \frac{u^{p+1}}{p+1}} d u\right) e^{\mathcal{B}_{0} \frac{\tilde{x}_{0}^{p+1}}{p+1}} \\
& \leq\left|\mathcal{Q}_{0}\right|\left(\int_{0}^{\infty} e^{\mathcal{B}_{0} \frac{u^{p+1}}{p+1}} d u\right) e^{\mathcal{B}_{0} \frac{\tilde{x}_{0}^{p+1}}{p+1}} \\
& =\left|\mathcal{Q}_{0}\right| c_{0}\left(\left|\mathcal{B}_{0}\right|\right) e^{\mathcal{B}_{0} \frac{\tilde{x}_{0}^{p+1}}{p+1}} .
\end{aligned}
$$

Uma conseqüência destas estimativas é o seguinte.

Corolário 5.3.3 Seja $\tilde{y}(\tilde{x})$ uma solução de $R_{0,0}\left(\mathcal{Q}_{0}\right)$. Suponha que existe um ponto $\tilde{x}_{0} \in \mathbf{R}$, no intervalo de definição de $y(x)$ tal que a lista $\left[\mathcal{Q}_{0}, \mathcal{B}_{0}, \tilde{x}_{0}, \tilde{y}_{0}\right]$ satisfaz uma das seguintes condições

(i) Se $p+1$ é par, $\mathcal{B}_{0}<0$, e

a) $\left[\mathcal{Q}_{0}>0, \tilde{x}_{0} \leq 0\right]$, ou $\left[\mathcal{Q}_{0}<0, \tilde{x}_{0} \geq 0\right]$, e

$$
\frac{-1}{\lambda}<\tilde{y}\left(\tilde{x}_{0}\right)<\frac{1}{2 \lambda} e^{\mathcal{B}_{0} \frac{\tilde{x}_{0}^{p+1}}{p+1}}
$$

b) $\left[\mathcal{Q}_{0}<0, \tilde{x}_{0} \leq 0\right]$, ou $\left[\mathcal{Q}_{0}>0, \tilde{x}_{0} \geq 0\right]$, e

$$
\frac{-1}{2 \lambda} e^{\mathcal{B}_{0} \frac{\dot{x}_{0}^{p+1}}{p+1}}<\tilde{y}\left(\tilde{x}_{0}\right)<\frac{1}{\lambda}
$$

(ii) Se $p+1$ é ímpar, $e$

a) $\left[\mathcal{Q}_{0}>0, \mathcal{B}_{0}>0, \tilde{x}_{0} \leq 0\right]$, ou $\left[\mathcal{Q}_{0}<0, \mathcal{B}_{0}<0, \tilde{x}_{0} \geq 0\right]$, e

$$
\frac{-1}{\lambda}<\tilde{y}\left(\tilde{x}_{0}\right) \leq 0 \text {; }
$$

b) $\left[\mathcal{Q}_{0}<0, \mathcal{B}_{0}>0, \tilde{x}_{0} \leq 0\right]$, ou $\left[\mathcal{Q}_{0}>0, \mathcal{B}_{0}<0, \tilde{x}_{0} \geq 0\right]$, e

$$
0 \leq \tilde{y}\left(\tilde{x}_{0}\right)<\frac{1}{\lambda}
$$


Então, a solução $\tilde{y}(\tilde{x})$ não pode escapar ao infinito em tempo finito.

\section{DemonstraçÃo}

(i) - Do lema 5.3.1, vale a relação

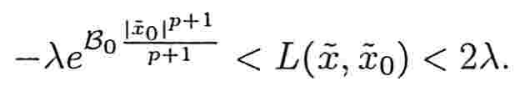

Se, $L\left(\tilde{x}, \tilde{x}_{0}\right)=\tilde{y}_{0}^{-1} e^{\mathcal{B}_{0} \frac{\bar{x}_{0}^{p+1}}{p+1}}$, vamos precisar ter

$$
\tilde{y}_{0}^{-1} e^{\mathcal{B}_{0} \frac{\dot{x}_{0}^{p+1}}{p+1}}<-\lambda e^{\mathcal{B}_{0} \frac{\dot{x}_{0}^{p+1}}{p+1}}, \quad 2 \lambda<\tilde{y}_{0}^{-1} e^{\mathcal{B}_{0} \frac{\bar{x}_{0}^{p+1}}{p+1}} .
$$

Daqui que obtemos

$$
\frac{-1}{\lambda}<\tilde{y}\left(\tilde{x}_{0}\right)<\frac{1}{2 \lambda} e^{\mathcal{B}_{0} \frac{\tilde{x}_{0}^{p+1}}{p+1}}
$$

onde $\tilde{y}\left(\tilde{x}_{0}\right)=\tilde{y}_{0}$

- Do lema 5.3.1, vale a relação

$$
-2 \lambda<L\left(\tilde{x}, \tilde{x}_{0}\right)<\lambda e^{\mathcal{B}_{0} \frac{\tilde{x}_{0}^{p+1}}{p+1}} .
$$

Aqui, se $L\left(\tilde{x}, \tilde{x}_{0}\right)=\tilde{y}_{0}^{-1} e^{\mathcal{B}_{0} \frac{\tilde{x}_{0}^{p+1}}{p+1}}$, vamos precisar ter

$$
\tilde{y}_{0}^{-1} e^{\mathcal{B}_{0} \frac{\tilde{x}_{0}^{p+1}}{p+1}}<-2 \lambda, \quad \lambda e^{\mathcal{B}_{0} \frac{\tilde{x}_{0}^{p+1}}{p+1}}<\tilde{y}_{0}^{-1} e^{\mathcal{B}_{0} \frac{\bar{x}_{0}^{p+1}}{p+1}} .
$$

Daqui decorre (i).

(ii) - Do lema 5.3.2, vale

$$
L\left(\tilde{x}, \tilde{x}_{0}\right) \geq-\lambda e^{\mathcal{B}_{0} \frac{\tilde{x}_{0}^{p+1}}{p+1}} .
$$

Logo vamos precisar ter as desigualdades

$$
0 \geq \tilde{y}_{0} e^{-\mathcal{B}_{0} \frac{\dot{x}_{0}^{p+1}}{p+1}}>\frac{-1}{\lambda} e^{-\mathcal{B}_{0} \frac{\dot{x}_{0}^{p+1}}{p+1}} .
$$


- Do lema 5.3.2, vale

$$
L\left(\tilde{x}, \tilde{x}_{0}\right) \leq \lambda e^{\mathcal{B}_{0} \frac{x_{0}^{p+1}}{p+1}} .
$$

Logo vamos precisar ter as desigualdades

$$
0 \leq \tilde{y}_{0} e^{-\mathcal{B}_{0} \frac{\bar{x}_{0}^{p+1}}{p+1}}<\frac{1}{\lambda} e^{-\mathcal{B}_{0} \frac{\dot{x}_{0}^{p+1}}{p+1}} .
$$

Daqui decorre (ii), e portanto é mostrado o corolário.

\subsection{Caso $R_{A, B}\left(\mathcal{Q}_{0}\right)$}

Aqui enfatizaremos o caso $\left[p+1\right.$ par; $\left.\mathcal{B}_{0}>0\right]$, pois este é o caso mais relevante para o trabalho. Definindo $\mathcal{B}_{+}:=\mathcal{B}_{0}$, e $\mathcal{B}_{-}:=(-1)^{p} \mathcal{B}_{0}$, o caso da transição $(s, u)$ é equivalente a ter $\mathcal{B}_{0}>0$ e $p+1$ par.

Neste caso consideremos a família não linear de equações diferenciais ordinárias

$$
R_{A, B}\left(\mathcal{Q}_{0}\right): \quad \frac{d y}{d x}=\left(\sum_{i=0}^{2 p-1} A_{i} x^{i}\right)+\left(\sum_{j=0}^{p-1} B_{j} x^{j}+\mathcal{B}_{0} x^{p}\right) y+\mathcal{Q}_{0} y^{2} .
$$

Seja $U \in\left(\mathbb{R}^{3 p}, 0\right)$, uma vizinhança aberta de $(A, B)=(0,0)$, e suponha que, para cada $(A, B) \in U$, associamos duas soluções da família Riccati $R_{A, B}\left(\mathcal{Q}_{0}\right)$

$$
y_{-}^{(A, B)}: U_{-\infty} \rightarrow \mathbb{R}, \quad \text { e } \quad y_{+}^{(A, B)}: U_{\infty} \rightarrow \mathbb{R}
$$

tais que :

i) $U_{-\infty}, U_{\infty} \subset \mathbb{R}$, são vizinhanças de $-\infty, e+\infty$ respectivamente;

ii) $y_{+}^{(A, B)}(x)$, e $y_{-}^{(A, B)}(x)$ dependem continuamente sobre $(A, B) \in U$;

iii) Estas soluções tem o comportamento assintótico

$$
\lim _{X \rightarrow 0} X^{p} y_{+}^{(A, B)}\left(\frac{1}{X}\right)=\lim _{X \rightarrow 0} X^{p} y_{-}^{(A, B)}\left(\frac{-1}{X}\right)=0,
$$

para cada $(A, B) \in U$; ou equivalentemente $y_{-}^{(A B)}(x)=o\left(x^{p}\right)=$ $y_{+}^{(A, B)}(x)$, sobre $U_{-\infty}, U_{\infty}$ respectivamente (ver Figura 5.1). 
Lema 5.4.1 Suponha que $\mathcal{B}_{-}<0$ (resp. $\left.\mathcal{B}_{+}>0\right)$. Então dada qualquer constante $K>0$, arbitrariamente grande, existe uma pequena vizinhança $N=N(K) \subset U$ de $(A, B)=(0,0)$, tal que para cada $(A, B) \in N$, a solução $y_{-}^{(A, B)}$ (resp. $y_{+}^{(A, B)}$ ) pode ser estendida ao intervalo $(-\infty, K]$ (resp. $[-K, \infty))$.

\section{DEMONSTRAÇÃo}

Os lemas da seção anterior mostram que as soluções $y_{-}^{(A, B)}$, e $y_{+}^{(A, B)}$, são únicas; realmente para $\mathcal{B}_{-}<0$, a solução $y_{-}^{(A, B)}$ pode ser vista como intersecção da variedade central $W_{-}$com o divisor excepcional $D$, ou seja

$$
y_{-}^{(A, B)}=W_{-} \cap D .
$$

(Estes fatos serão precisados no próximo capítulo).

Note que para $(A, B)=(0,0), y(x) \equiv 0$ é uma solução de $R_{A, B}\left(\mathcal{Q}_{0}\right)$. Assim pela unicidade temos

$$
y_{-}^{(0,0)}(x) \equiv 0, \quad \forall x \in \mathbb{R}
$$

Daqui o resultado segue da dependência contínua das variedades centrais sobre os parâmetros $(A, B)$ (o resultado para $y_{+}^{(A, B)}$, é análogo).

Este resultado é estendido como segue.

Lema 5.4.2 Seja $y_{-}^{(A, B)}$ e $y_{+}^{(A, B)}$ como em (5.14). Suponha que existe um ponto $x_{0} \in U_{-\infty}$ (resp. $x_{0} \in U_{\infty}$ ), tal que o valor de $y_{-}^{(A, B)}$ (resp. $y_{+}^{(A, B)}$ ) para $x=x_{0}$, e $A=B=0$

$$
y\left(x_{0}\right):=y_{-}^{(0,0)}\left(x_{0}\right),\left(\text { resp. } \quad y\left(x_{0}\right):=y_{+}^{(0,0)}\left(x_{0}\right)\right)
$$

verifica uma das condições do corolário 5.3.3, da seção anterior. Então, para cada constante $K>0$, arbitrariamente grande, existe uma pequena vizinhança $N=N(K) \subset U$ de $(A, B)=(0,0)$ tal que, para cada $(A, B) \in N$, a solução $y_{-}^{(A, B)}(x)$, (resp. $\left.y_{+}^{(A, B)}(x)\right)$, pode ser estendida a uma solução de $R_{A, B}\left(\mathcal{Q}_{0}\right)$ a qual é definida para todo $x \in(-\infty, K]$ (resp. $x \in[-K, \infty)$ ).

\section{DemonstraçÃo}

Supondo que tal vizinhança $N$ não existe, a continuidade de $y_{ \pm}^{(A \cdot B)}(x)$, respeito a $(A, B)$ implicaria que $y_{ \pm}^{(0,0)}(x)$ escapa ao infinito em tempo finito, isto contradiz o corolário 5.3.3; por tanto este resultado é imediata conseqüência dos lemas da seção anterior. 


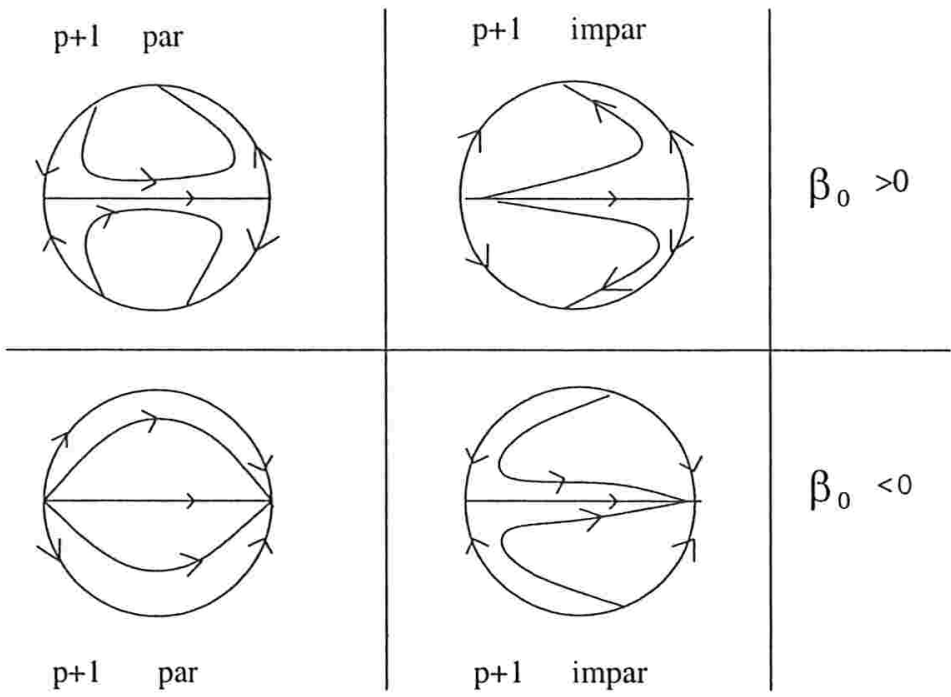

Figura 5.1: Compactificações sobre o Divisor Excepcional $\tilde{D}$.

Pela fórmula de variação das constantes, a solução geral do problema de Cauchy $\left[R_{A, B}\left(\mathcal{Q}_{0}\right), y_{0}\right]$ pode ser dada implicitamente por

$$
y(x)=e^{P(x)}\left(y_{0}+\int_{0}^{x} Q(t) e^{-P(t)} d t+\mathcal{Q}_{0} \int_{0}^{x} e^{-P(t)} y(t)^{2} d t\right)
$$

onde

$$
\begin{aligned}
y_{0} & =y\left(x_{0}\right) \\
Q(x) & =\sum_{i=0}^{2 p-1} A_{i} x^{i} \\
P(x) & =B_{0} x+B_{1} \frac{x^{2}}{2}+\ldots+B_{p-1} \frac{x^{p}}{p}+\mathcal{B}_{0} \frac{x^{p+1}}{p+1} .
\end{aligned}
$$

Considerando o caso $(s, u),\left(\mathcal{B}_{-}<0, \mathcal{B}_{+}>0\right)$, do lema 5.4.1, para $K=0$, existe uma pequena vizinhança $N \subset\left(\mathbb{R}^{3 p}, 0\right)$, de $(A, B)=(0,0)$ tal que, para cada $(A . B) \in N$, existe soluções únicas $y_{-}^{(A, B)}$ e $y_{+}^{(A, B)}$, definidas para todo $x \in(-\infty, 0]$ e $x \in[0, \infty)$ respectivamente, com os comportamentos assintóticos (5.15). 
Assim como no caso linear, aqui podemos considerar a função distância

$$
\begin{aligned}
\delta: \quad & N \longrightarrow \mathbb{R} \\
& (A, B) \longmapsto y_{+}^{(A, B)}(0)-y_{-}^{(A, B)}(0) .
\end{aligned}
$$

Lema 5.4.3 No caso $(s, u)$, a função distância $\delta$ é definida sobre uma pequena vizinhança $N \subset\left(\mathbb{R}^{3 p}, 0\right)$ de $(A, B)=(0,0)$, além disso

$$
\frac{\partial \delta}{\partial A_{s}}=-\left[C_{s}(B)-(-1)^{s+1} C_{s}(\bar{B})\right]+O(A)
$$

para $s \in\{0,1, \ldots, 2 p-1\}$, onde $C_{s}(B)$ e $C_{s}(\bar{B})$ são definidas no lema 5.2.1.

\section{DEMONSTRAÇÃo}

Definamos as seguintes quantidades

$$
\begin{aligned}
& I_{+}(A, B)=\int_{0}^{\infty} e^{-P(x)} y_{+}^{(A, B)}(x)^{2} d x, \\
& I_{-}(A, B)=\int_{0}^{-\infty} e^{-P(x)} y_{-}^{(A, B)}(x)^{2} d x .
\end{aligned}
$$

Como $y_{+}^{(A, B)}, \quad y_{-}^{(A, B)} \in C^{\infty}(N)$ (pelos lemas da seção anterior), então também temos que $I_{+}, I_{-} \in C^{\infty}(N)$.

Da solução geral dada em (5.16), usando a compactificação $x=\frac{1}{X}, \quad y=\frac{Y}{X^{p}}$ e fazendo $X \rightarrow 0$, obtemos

$$
0=y_{+}^{(A, B)}(0)+\sum_{k=0}^{2 p-1} C_{k}(B) A_{k}+\mathcal{Q}_{0} I_{+}(A, B) .
$$

Similarmente obtemos

$$
0=y_{-}^{(A, B)}(0)+\sum_{k=0}^{2 p-1}(-1)^{k+1} C_{k}(\bar{B}) A_{k}+\mathcal{Q}_{0} I_{-}(A, B) .
$$

Como $y_{+}^{(0, B)}(x)=y_{-}^{(0, B)}(x)=0$, então temos $I_{+}(0, B)=I_{-}(0, B)=0$, e conseqüentemente vale

$$
\nabla I_{+}(0, B)=\left(\frac{\partial I_{+}}{\partial A_{0}}, \ldots, \frac{\partial I_{+}}{\partial A_{2 p-1}}, \frac{\partial I_{+}}{\partial B_{0}}, \ldots, \frac{\partial I_{+}}{\partial B_{p-1}}\right)_{(0, B)}=(0, \ldots, 0) .
$$

Assim, subtraindo (5.18) de (5.17), e derivando com respeito a $A_{s}$, para $s \in\{0,1, \ldots, 2 p-1\}$, obtemos

$$
\frac{\partial \delta}{\partial A_{s}}=-\left[C_{s}(B)-(-1)^{s+1} C_{s}(\bar{B})\right]+O(A) .
$$


80 CAPÍTULO 5. DINÂMICA ASSINTÓTICA SOBRE A CARTA $K_{\bar{\varepsilon}}$

Observação 5.4.4 Como valem as igualdades,

$$
C_{s}(B=0)=C_{s}(\bar{B}=0)=c_{s}\left(\mathcal{B}_{0}\right)
$$

então temos a expressão particular

$$
\left.\frac{\partial \delta}{\partial A_{s}}\right|_{B=0}=-2 c_{s}\left(\mathcal{B}_{0}\right)+O(A)
$$

para todos os indice pares $s=0,2, \ldots, 2 p-2$. 


\section{Capítulo 6}

\section{Dinâmica sobre as cartas $K_{ \pm} \bar{x}_{\varepsilon}$}

Aqui vamos estudar as variedades centrais $W_{ \pm}$próximo ao divisor excepcional $\hat{D}_{0}=\hat{D} \cap\left\{\mathcal{A}_{r}=0\right\}$. Escrevamos as coordenadas da carta $K_{ \pm \bar{x}_{\varepsilon}}$ simplesmente

$$
\left(x, y, e, A, B, \mathcal{A}_{r}\right) \in U_{\hat{x}} \times U_{\hat{y}} \times U_{e} \times U_{A, B} \times U_{\mathcal{A}_{r}},
$$

onde

$$
\begin{cases}U_{\hat{x}} & \in\left(\mathbb{R}^{+}, 0\right) \\ U_{\hat{y}} & =\mathbb{R} \\ U_{e} & =\mathbb{R}^{+} \\ U_{A, B} & \in\left(\mathbb{R}^{3 p}, 0\right) \\ U_{\mathcal{A}_{r}} & \in\left(\mathbb{R}^{n-3 p}, 0\right) .\end{cases}
$$

Nestas coordenadas, o campo $\bar{X}^{ \pm}$, é dado por

$$
\bar{X}^{ \pm}(x, y, e, \mathcal{A})= \pm e^{p+1}\left(x \frac{\partial}{\partial x}-e \frac{\partial}{\partial e}\right)+\hat{F}^{ \pm}(x, y, e, \mathcal{A}) \frac{\partial}{\partial y},
$$

$\operatorname{com} \hat{F}^{ \pm}(x, y, e, \mathcal{A})=\hat{F}_{0}^{ \pm}(x, e, \mathcal{A})+\hat{F}_{1}^{ \pm}(x, e, \mathcal{A}) y+\hat{Q}^{ \pm}(x, y, e, \mathcal{A}) y^{2}$, definido como

$$
\begin{aligned}
\hat{F}_{0}^{ \pm}(x, e, \mathcal{A}) & =\sum_{i=0}^{2 p-1}( \pm 1)^{i} A_{i} e^{2 p-i}+O(x), \\
\hat{F}_{1}^{ \pm}(x, e, \mathcal{A}) & =\mp p e^{p+1}+\sum_{j=0}^{p-1}( \pm 1)^{j} B_{j} e^{p-j}+( \pm 1)^{p} \mathcal{B}\left(\mathcal{A}_{r}\right)+O(x), \\
\hat{Q}^{ \pm}(x, y, e, \mathcal{A}) & =Q\left(\mathcal{A}_{r}\right)+O(x) .
\end{aligned}
$$


onde $\mathcal{B}_{0}=\mathcal{B}(0) \neq 0, \mathcal{Q}_{0}=\mathcal{Q}(0)$ e $O(x)$ denotam funções analíticas divisíveis por $x$.

Para cada parâmetro fixado $\mathcal{A}_{0}=\left(A, B, \mathcal{A}_{r}\right)$, em uma vizinhança suficientemente pequena de $\mathcal{A}_{r}=0$ os pontos

$$
p_{ \pm}^{\mathcal{A}_{0}}=\left\{x=y=e=0, \mathcal{A}=\mathcal{A}_{0}\right\}
$$

são singularidades normalmente hiperbólicas para o campo $\bar{X}^{ \pm}$, pois a matriz Jacobiana $D \bar{X}^{+}\left(p_{+}^{\mathcal{A}_{0}}\right)$, (resp. $D \bar{X}^{-}\left(p_{-}^{\mathcal{A}_{0}}\right)$ ) tem um autovalor não nulo

$$
\mathcal{B}_{+}\left(\mathcal{A}_{r}\right):=\mathcal{B}\left(\mathcal{A}_{r}\right),\left(\text { resp. } \quad \mathcal{B}_{-}\left(\mathcal{A}_{r}\right):=(-1)^{p} \mathcal{B}\left(\mathcal{A}_{r}\right)\right) \text {. }
$$

Denotaremos as constantes não nulas $\mathcal{B}_{+}:=\mathcal{B}_{+}(0), \mathcal{B}_{-}:=\mathcal{B}_{-}(0)$. Logo, o conjunto

$$
\operatorname{Sing}\left(\overline{X^{ \pm}}\right)=\left\{\left(x, y, e, A, B, \mathcal{A}_{r}\right) \mid x=y=e=0\right\}:=P_{ \pm} \approx U_{A, B} \times U_{\mathcal{A}_{r}}
$$

é uma subvariedade de codimensão 3, constituída inteiramente de singularidades normalmente hiperbólicas.

O teorema da variedade central, implica a existência de variedades centrais em cada ponto de $P_{ \pm}$; em geral estas variedades não são únicas. Mais precisamente temos o seguinte resultado

Proposição 6.0.5 Para cada natural $k \in \mathrm{N}$, e cada ponto fixo $p_{ \pm} \in P_{ \pm}$, existem vizinhanças $V_{ \pm} \subset U_{\hat{x}, e, A, B, \mathcal{A}_{r}}$ destes pontos e funções $C^{k}$

$$
\begin{array}{ll}
w_{ \pm}: & V_{ \pm} \longrightarrow \mathbf{R} \\
& (x, e, \mathcal{A}) \longmapsto y=w_{ \pm}(x, e, \mathcal{A})
\end{array}
$$

tais que:

(i) $w_{ \pm}(x, 0,0)=0$,

(ii) $W_{ \pm}=\operatorname{graf}\left\{y=w_{ \pm}(x, e, \mathcal{A})\right\}$, são variedades localmente invariantes pelo fluxo de $\bar{X}^{ \pm}$.

Todas estas variedades satisfazem a seguinte propriedade de unicidade.

Lema 6.0.6 Suponha que $\mathcal{B}_{+}>0$, (resp. $\left.\mathcal{B}_{-}<0\right)$. Então a intersecção de qualquer variedade central local $W_{+}\left(\right.$resp. $\left.W_{-}\right)$, de classe $C^{k}$ com o divisor excepcional $D_{0}=D \cap\left\{\mathcal{A}_{r}=0\right\}$

$W_{+} \cap D_{0}=\operatorname{graf}\left\{y=w_{+}(0, e, \mathcal{A})\right\},\left(\right.$ resp. $\left.W_{-} \cap D_{0}=\operatorname{graf}\left\{y=w_{-}(0, e, \mathcal{A})\right\}\right)$

é única e de classe $C^{\infty}$. 
DEmonstração

A variedade $c:=W_{ \pm} \cap D_{0}$ é uma variedade central local de classe $C^{k}$ para o campo restrito

$$
\left.\bar{X}^{ \pm}\right|_{D_{0}}=\mp e^{p+2} e \frac{\partial}{\partial e}+\hat{F}^{ \pm}(0, y, e, A, B, 0) \frac{\partial}{\partial y} .
$$

onde

$\hat{F}^{ \pm}=\left[\sum_{i=0}^{2 p-1}( \pm 1)^{i} A_{i} e^{2 p-i}\right]+\left[\mp p e^{p+1}+\sum_{j=0}^{p-1}( \pm 1)^{j} B_{j} e^{p-j}+( \pm 1)^{p} \mathcal{B}_{0}\right] y+\mathcal{Q}_{0} y^{2}$.

A unicidade decorre do teorema $(3.2)$ em $[J S]$, pois $\mathcal{B}_{+}>0$ (resp. $\left.\quad \mathcal{B}_{-}<0\right)$. O fato de que $c \in C^{\infty}$ decorre do teorema (5.1) em [JS].

Consideremos agora expansões formais em séries de cada variedade central sobre $P_{ \pm}$.

Lema 6.0.7 Dado um subconjunto aberto arbitrário $V_{\mathcal{A}}=V_{A, B} \times V_{\mathcal{A}_{r}} \subset$ $U_{A, B} \times U_{\mathcal{A}_{r}}$, tal que $V_{A, B} \in\left(\mathbb{R}^{3 p}, 0\right)$ tem fecho compacto e $V_{\mathcal{A}_{r}} \in\left(\mathbb{R}^{n-3 p}, 0\right)$, existem intervalos $V_{x}^{ \pm}=\left[0, x_{ \pm}\right) \subset \mathbb{R}^{+},\left(x_{+}, x_{-}>0 \in U_{\hat{x}}\right.$, dependem sobre $V_{\mathcal{A}}$ ) e existem séries formais únicas

$$
\widehat{W}_{ \pm}(x, e, \mathcal{A})=\sum_{i=0}^{\infty} w_{i}^{ \pm}(x, \mathcal{A}) e^{i}
$$

definidas por uma coleção de funções analíticas

$$
w_{i}^{ \pm} \in C^{w}\left(V_{x}^{ \pm} \times V_{\mathcal{A}}\right), \quad i \in \mathbf{N}
$$

tais que para cada ponto $p_{ \pm} \in P_{ \pm} \cap V_{\mathcal{A}}$, e cada variedade central local $W_{ \pm}=$ graf $\left\{y=w_{ \pm}(x, e, \mathcal{A})\right\}$, definida numa vizinhança $U$ deste ponto,

$$
w_{ \pm} \in C_{\text {flat }}^{k}\left(U,\{e=0\}, \widehat{W}_{ \pm}\right) .
$$

\section{DEmONSTRAÇÃo}

Como $\mathcal{B}_{+}\left(\mathcal{A}_{r}\right) \neq 0$, a demonstração é similar à proposição 3.2.1. Os argumentos sobre $P_{-}$, são análogos. 
Definição 6.0.8 Dado um ponto arbitrário $x_{0} \in V_{x}^{ \pm}$, definimos as restrições

$$
\widehat{W}_{ \pm, x_{0}}:=\sum_{i=0}^{\infty} w_{i}^{ \pm}\left(x_{0}, \mathcal{A}\right) e^{i}
$$

da variedade central formal $\widehat{W}_{ \pm}$.

\subsection{Região atratora}

Para à construção das Variedades Centrais Dinâmicas sobre $P_{ \pm}$, precisamos introduzir o conceito de função condição inicial:

i) Suponha que $\mathcal{B}_{+}>0$.

Fixando um subconjunto aberto arbitrário $V_{\mathcal{A}} \subset U_{\mathcal{A}}$ como no lema 6.0.7, escolhamos um ponto $x_{0}>0 \in V_{x}^{+}$, e um intervalo $V_{e}=\left(0, e_{0}\right)$, para algum $e_{0}>0 \in U_{e}$.

Uma função

$$
\begin{aligned}
i^{+}: & V_{e} \times V_{\mathcal{A}} \longrightarrow \mathbf{R} \\
& (e, \mathcal{A}) \longmapsto y=i^{+}(e, \mathcal{A})
\end{aligned}
$$

de classe $C^{\infty}$, tal que

$$
i^{+} \in C_{\text {flat }}^{\infty}\left(V_{e} \times V_{\mathcal{A}},\{e=0\}, \widehat{W}_{+, x_{0}}\right)
$$

será dita uma função condição inicial para $\bar{X}^{+}$sobre $P_{+}$.

ii) Suponha agora que $\mathcal{B}_{+}<0$.

Fixemos um ponto $e_{0}>0 \in U_{e}$. A fim de definir a função condição inicial, necessitamos do seguinte resultado:

Lema 6.1.1 Sobre as hipóteses acima, existem constantes $e_{+}, y_{+}>0$, dependendo somente sobre o subconjunto $V_{\mathcal{A}}$, tal que para cada ponto dado

$$
q_{0}=\left\{(x, y, e, \mathcal{A})=\left(0, y_{0}, e_{0}, \mathcal{A}\right)\right\} \in D,
$$

onde $0<e_{0}<e_{+},\left|y_{0}\right|<y_{+}, \quad \mathcal{A} \in V_{\mathcal{A}}$,

a órbita do campo $\left.\bar{X}^{+}\right|_{D}$ : começando em $q_{0}$ tem seu $\omega$-limite em $P_{+}$. 


\section{DEmonstraÇÃo}

Sobre o divisor excepcional $D=\{x=0\}$, o campo $\left.\bar{X}^{+}\right|_{D}$ é dado por

$$
\left.\bar{X}^{+}\right|_{D}=-e^{p+2} \frac{\partial}{\partial e}+\hat{F}^{+}(0, y, e, \mathcal{A}) \frac{\partial}{\partial y} .
$$

Como $\mathcal{B}_{+}<0$, para cada $p \in P_{+}$, pelo corolário (13.3) dado em $[D P]$ temos que existe uma $C^{1}$ - mudança de coordenadas definida sobre uma vizinhança $U_{p}$ de $p$ tal que $\left.\bar{X}^{+}\right|_{D}$ é $C^{1}$ - equivalente ao campo

$$
Y(y, e, \mathcal{A})=-y \frac{\partial}{\partial y}-e^{p+2} g(e, \mathcal{A}) \frac{\partial}{\partial e} .
$$

Para alguma função estritamente positiva $g(e, \mathcal{A})$ de classe $C^{1}$. Agora sobre a região $\{e=0\}$ é claro que a origem $(e, y)=(0,0)$ é $\omega$-limite para as órbitas de $Y$.

Por outro lado como $V_{\mathcal{A}}$ tem fecho compacto, podemos então cobrir $V_{\mathcal{A}}$ por um número finito de vizinhanças $U_{p}$ de $p \in P_{+}$. De fato podemos escolher as constantes $e_{+}, y_{+}$de tal maneira que a região

$$
R^{+}=\left\{(e, y)\left|0 \leq e<e_{+},\right| y \mid<y_{+}\right\}
$$

esteja contida na intersecção de todos os domínios $U_{p} \cap\{\mathcal{A}=$ const. $\}$

Diremos que o conjunto aberto $R^{+}$definido em (6.2), é a região atratora para campo vetorial restrito $\left.\bar{X}^{+}\right|_{D}$, relativamente ao conjunto $V_{\mathcal{A}}$ (ver Figura 6.1).

\section{Definição 6.1.2 Uma função}

$$
\begin{aligned}
i: & V_{x} \times V_{\mathcal{A}} \longrightarrow \mathbf{R} \\
& (x, \mathcal{A}) \longmapsto y=i(x, \mathcal{A})
\end{aligned}
$$

de classe $C^{\infty}$, definida sobre um subconjunto aberto $V_{x} \times V_{\mathcal{A}}$, onde $V_{\mathcal{A}} \subset U_{\mathcal{A}}$ tem fecho compacto e $V_{x}:=\left[0, x_{0}\right]$ para algum $x_{0}>0 \in V_{x}^{+}$, será chamada de função condição inicial, (para o caso $\mathcal{B}_{+}<0$ ) se

$$
\sup _{\mathcal{H} \in V_{\mathcal{A}}}\|i(0, \mathcal{A})\|<y_{+}
$$

Agora apresentamos os resultados que definem as Variedades Centrais Dinâmicas. 


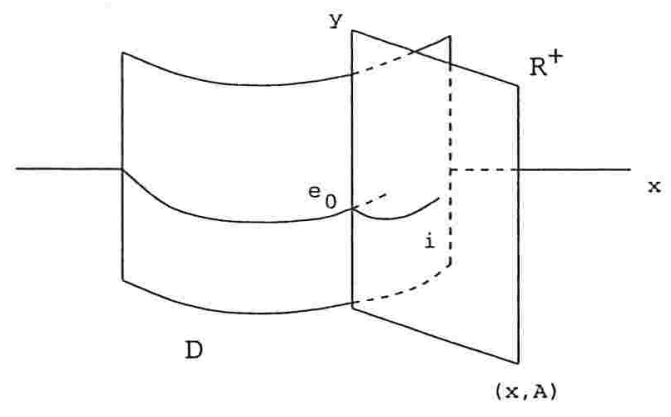

Figura 6.1: Região Atratora Na Carta $K_{+x_{\varepsilon}}$.

Proposição 6.1.3 Suponha que $\mathcal{B}_{+}>0$ e sejami $(e, \mathcal{A}) \in C_{\text {flat }}^{\infty}\left(V_{e} \times V_{\mathcal{A}},\{e=\right.$ $0\}, \widehat{W}_{+, x_{0}}$ ) uma função condição inicial arbitrária para $\bar{X}^{+}$e $N_{x}:=\left[0, x_{0}\right] \subset$ $\mathrm{R}^{+}$. Então existe um intervalo

$$
N_{e}:=\left(0, e_{1}\right) \subset V_{e}, \quad\left(0<e_{1} \leq e_{0}\right)
$$

e uma única função $C^{\infty} w_{+}(x, e, \mathcal{A})$ definida sobre o conjunto aberto $N^{+}=$ $N_{x} \times N_{e} \times V_{\mathcal{A}}$ que verifica as seguintes condições:

(i) $w_{+}\left(x_{0}, e, \mathcal{A}\right)=i(e, \mathcal{A}), \quad \forall(e, \mathcal{A}) \in N_{e} \times V_{\mathcal{A}}$,

(ii) $w_{+} \in C_{\text {flat }}^{\infty}\left(N^{+},\{e=0\}, \widehat{W}_{+}\right)$,

(iii) $W_{+}=\operatorname{graf}\left\{y=w_{+}(x, e, \mathcal{A})\right\}$, é uma variedade invariante por $\bar{X}^{+}$.

\section{DemonstraçÃo}

Consideremos o campo $-\bar{X}^{+}$, dado por

$$
-\bar{X}^{+}=-e^{p+1}\left(x \frac{\partial}{\partial x}-e \frac{\partial}{\partial e}\right)-\hat{F}^{+} \frac{\partial}{\partial y} .
$$

Como $\mathcal{B}_{+}>0$, pelo corolário 13.3 em $[D P]$, existe uma mudança de coordenadas $C^{k} \varphi:(x, y, e, \mathcal{A}) \longrightarrow\left(x^{\prime}, y^{\prime}, e^{\prime}, \mathcal{A}^{\prime}\right)$ definida sobre alguma vizinhança de $p \in P_{+}$, tal que $-\bar{X}^{+}$é $C^{k}$-equivalente a

$$
Y=-y \frac{\partial}{\partial y}-e^{p+1} G(x, e, \mathcal{A})\left(x \frac{\partial}{\partial x}-e \frac{\partial}{\partial e}\right),
$$

para alguma função $C^{k} G(x, e, \mathcal{A})$ estritamente positiva. 
- Para qualquer $p \in P_{+}$, consideremos uma Variedade central local de classe $C^{k} W^{\prime}=\operatorname{graf}\left\{y=w^{\prime}(x, e, \mathcal{A})\right\}$ definida sobre alguma vizinhança $U^{\prime}$ de $p \in P_{+}$e mostremos que $w_{+}-w^{\prime} \in C_{\text {flat }}^{\infty}\left(U^{\prime},\{e=0\}\right)$. De fato nas coordenadas $\left(x^{\prime}, y^{\prime}, e^{\prime}, \mathcal{A}^{\prime}\right)$ temos que $W^{\prime}=\operatorname{graf}\left\{y^{\prime}=0\right\}$ e que $-\bar{X}^{+}$é $C^{k}$-equivalente (eliminando as ",$\prime^{\prime \prime}$ ) a

$$
Z=-\left(\frac{1}{e^{p+1} G}\right) y \frac{\partial}{\partial y}-\left(x \frac{\partial}{\partial x}-e \frac{\partial}{\partial e}\right), \quad \text { sobre } \quad\{e>0\} .
$$

Agora consideramos a condição inicial

$$
\xi_{0}=\left\{(x, y, e, \mathcal{A})=\left(x_{0}, \tilde{i}(e, \mathcal{A}), e, \mathcal{A}\right)\right\}
$$

onde $\tilde{i}:=i \circ \varphi^{-1} \in C_{\text {flat }}^{\infty}\left(\{e>0\} \times V_{\mathcal{A}},\{e=0\}\right)$. A solução explicita do problema de Cauchy $\left[Z, \xi_{0}\right]$ é dada por

$$
Z_{t}\left(\xi_{0}\right)=\left\{\begin{array}{l}
e(t)=e \exp (-t) \\
x(t)=x_{0} \exp (t) \\
y(t)=\tilde{i}(e(t), \mathcal{A}) \exp \left(-\int_{0}^{t} \frac{d \tau}{e(\tau)^{p+1} G}\right) .
\end{array}\right.
$$

Tomando $t=\ln \left(\frac{x}{x_{0}}\right)$ e substituindo nas outras equações, temos a convergência $y \rightarrow 0, e \rightarrow 0$ juntamente com as derivadas $\frac{\partial^{k} y}{\partial \varepsilon^{k}}$; isto mostra (ii).

- Vamos considerar agora a saturação $\mathcal{W}$ do conjunto

$$
\xi=\bigcup_{(e, \mathcal{A}) \in V_{e} \times V_{\mathcal{A}}}\left\{(x, y, e, \mathcal{A}) \mid x=x_{0}, y=i(e, \mathcal{A})\right\}
$$

pelo fluxo $Z_{t}\left(\xi_{0}\right)$ de $Z \equiv Y \equiv-\bar{X}^{+}$.

Logo daqui existem os domínios requeridos $N_{x}, N_{e}$ como acima, tal que a restrição de $\mathcal{W}$ a $N^{+}=N_{x} \times N_{e} \times V_{\mathcal{A}}$ é definida como o gráfico de uma função $w_{+} \in C^{\infty}\left(N^{+}\right)$; daqui são satisfeitas $(i)$, (iii).

Para $\mathcal{B}_{+}<0$, temos o resultado análogo.

Proposição 6.1.4 Suponha que $\mathcal{B}_{+}<0$, seja i $(x, \mathcal{A})$ uma função condição inicial arbitrária para $\bar{X}^{+}$(como na definição 6.1.2), escolhemos um ponto 
$e_{0} \in\left(0, e_{+}\right)$arbitrário $\left(e_{+}\right.$, definido na região atratora $\left.R^{+}\right)$e seja $N_{e}:=$ $\left[0, e_{0}\right) \subset \mathbf{R}^{+}$. Então existe um intervalo

$$
N_{x}=\left[0, e_{1}\right) \subset V_{x},\left(0<x_{1} \leq x_{0}\right)
$$

e uma única $C^{\infty}$-função $w_{+}(x, e, \mathcal{A})$ definida sobre o conjunto aberto $N^{+}=$ $N_{x} \times N_{e} \times V_{\mathcal{A}}$ tal que

(i) $w_{+}\left(x, e_{0}, \mathcal{A}\right)=i(x, \mathcal{A}), \quad \forall(x, \mathcal{A}) \in N_{x} \times V_{\mathcal{A}}$,

(ii) $w_{+} \in C_{\text {flat }}^{\infty}\left(N^{+},\{e=0\}, \widehat{W}_{+}\right)$,

(iii) $W_{+}=\operatorname{graf}\left\{y=w_{+}(x, e, \mathcal{A})\right\}$, é uma variedade invariante por $\bar{X}^{+}$.

(Veja Figura 6.2)

Observação 6.1.5 - De forma análoga, definimos $R^{-}$e $W_{-}$sobre $P_{-}$; assim as variedades invariantes $W_{-}, W_{+}$construídas no processo acima serão ditas de Variedades Centrais Dinâmicas locais sobre $P_{-}, P_{+}$.

- A classificação das singularidades degeneradas dada na seção 2.2.1 relaciona-se com os sinais de $\mathcal{B}_{-}$e $\mathcal{B}_{+}$da seguinte forma:

$-\mathcal{B}_{-}>0, \mathcal{B}_{+}>0$, se e somente se $0 \in \Gamma$ esta no caso $(u, u)$

$-\mathcal{B}_{-}<0, \mathcal{B}_{+}>0$, se e somente se $0 \in \Gamma$ esta no caso $(s, u)$

$-\mathcal{B}_{-}>0, \mathcal{B}_{+}<0$, se e somente se $0 \in \Gamma$ esta no caso $(u, s)$

$-\mathcal{B}_{-}<0, \mathcal{B}_{+}<0$, se e somente se $0 \in \Gamma$ esta no caso $(s, s)$

De fato, nas cartas $K_{ \pm x_{\varepsilon}}$, temos que os conjuntos $P_{-}, P_{+}$, estão sobre a fronteira da contra imagem do blowing-up $\Psi^{-1}(\Gamma)$; logo por continuidade o sinal de $\mathcal{B}_{-}$(resp. $\mathcal{B}_{+}$) corresponde ao sinal do autovalor $\mathcal{B}_{x} \neq 0$ em cada um dos pontos não degenerados $x \in \Gamma \cap\{x<0\}$ (resp. $x \in \Gamma \cap\{x>0\}$ ). 


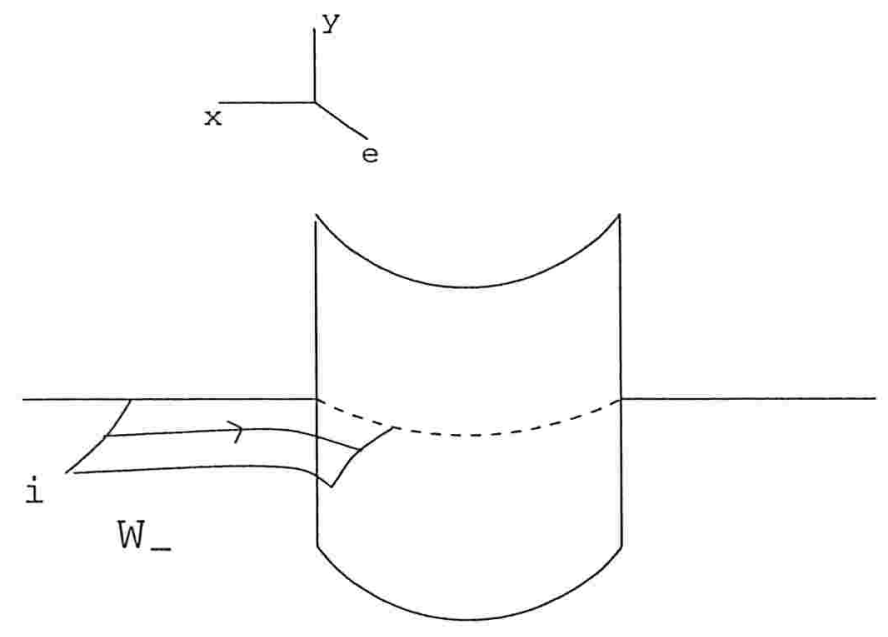

$\mathrm{D}$

Figura 6.2: Variedade Central Dinâmica na Carta $K_{-x_{\varepsilon}}$.

\subsection{Extensão Dinâmica das variedades Centrais}

Sobre $P_{-}$, consideremos uma variedade central local arbitraria $W_{-}$, definida sobre o domínio $N^{-}=N_{x} \times N_{e} \times V_{\mathcal{A}}$ por

$$
W_{-}=\operatorname{graf}\left\{y=w_{-}(x, e, \mathcal{A})\right\}
$$

Observamos que, se fixamos um ponto arbitrário $e_{1}>0 \in N_{e}$, a intersecção $W_{-} \cap\left\{e=e_{1}\right\}$ é dada pela subvariedade de codimensão 2

$$
\hat{\xi}:=\bigcup_{(x, \mathcal{A}) \in N_{x} \times V_{\mathcal{A}}}\left\{(x, y, e, \mathcal{A})=\left(x, w_{-}\left(x, e_{1}, \mathcal{A}\right), e_{1}, \mathcal{A}\right)\right\} .
$$

Como $e_{1}>0$, esta subvariedade está contida no domínio $U_{\bar{\varepsilon}}=\{\bar{\varepsilon}>0\}$ da carta $K_{\bar{\varepsilon}}$. Na relação feita desta carta com a carta $K_{-\bar{x}_{\bar{\varepsilon}}}$ no capítulo 4 , aparecem as igualdades de compactificação

$$
\left\{\begin{array}{lll}
\tilde{x} & =\frac{-1}{e} \\
\tilde{y} & =\frac{\tilde{y}}{e^{p}} \\
\tilde{\Xi} & =\hat{x} e \\
\left(A_{i}, B_{j}, \mathcal{A}_{r}\right) & =\left(A_{i}, B_{j}, \mathcal{A}_{r}\right) .
\end{array}\right.
$$


Daqui $\hat{\xi}$ pode ser escrito como o conjunto

$$
\tilde{\xi}:=\bigcup_{(\tilde{\varepsilon}, \mathcal{A}) \in N_{\tilde{\varepsilon}} \times V_{\mathcal{A}}}\left\{(\tilde{x}, \tilde{y}, \tilde{\varepsilon}, \mathcal{A})=\left(\frac{-1}{e_{1}}, H(\tilde{\varepsilon}, \mathcal{A}), \tilde{\varepsilon}, \mathcal{A}\right)\right\},
$$

onde

$$
\begin{aligned}
N_{\tilde{\varepsilon}} & =\left\{\tilde{\varepsilon} \in \mathrm{R}^{+} \mid \frac{\tilde{\varepsilon}}{e_{1}} \in N_{x}\right\}, \\
H(\tilde{\varepsilon}, \mathcal{A}) & :=e_{1}^{-p} w_{-}\left(\frac{\tilde{\varepsilon}}{e_{1}}, e_{1}, \mathcal{A}\right) .
\end{aligned}
$$

Sobre a carta $K_{\bar{\varepsilon}}$, o campo $\bar{X}$ é dado pela família analítica de equações diferenciais de primeira ordem

$$
\frac{d \tilde{y}}{d \tilde{x}}=\tilde{F}(\tilde{x}, \tilde{y}, \tilde{\varepsilon}, \mathcal{A}) .
$$

Para cada $q \in \tilde{\xi}$, seja $\tilde{y}=\phi(\tilde{x}, q)$ a solução analítica do problema de valor inicial $[\bar{X}, q]$, definida sobre seu intervalo maximal $I(q)=(-\infty, \tilde{x}(q))$, que depende continuamente de $q$.

Definição 6.2.1 Usando o fluxo de $\bar{X}$, definamos o saturado de $\tilde{\xi}$ em $U_{\bar{\varepsilon}}$ dado por

$$
\mathcal{W}^{-}:=\bigcup_{q \in \tilde{\xi}, \tilde{x} \in I(q)}\{\tilde{y}=\phi(q, \tilde{x})\}
$$

Diremos que $\mathcal{W}^{-}$é a extensão da Variedade Central local $W_{-}$. (Ver Figura 6.3)

Neste sentido, a dependência contínua das soluções sobre as condições iniciais mostra o seguinte resultado de extensão sobre $I(q)$.

Proposição 6.2.2 Existe um subconjunto aberto (possivelmente vazio) $\mathcal{O}^{-} \subset$ $N_{\tilde{\varepsilon}} \times V_{\mathcal{A}}$ tal que para cada ponto $\left.q \in \tilde{\xi}\right|_{\mathcal{O}^{-}}$, o correspondente intervalo maximal de definição $I(q)$ contem a origem $\tilde{x}=0$.

Assim, chamaremos a $\mathcal{O}^{-}$o domínio maximal de extensão da variedade central local $W_{-}$. Geometricamente temos que cada curva solução começando em $\left.\tilde{\xi}\right|_{\mathcal{O}^{-}}$corta a seç̧ão transversal $\Sigma:=\{\tilde{x}=0\}$ num único ponto $q^{\prime} \in \Sigma$.

Neste sentido a correspondência $C^{\infty} \varphi:(\tilde{\varepsilon}, \mathcal{A}) \longmapsto\left(\frac{-1}{e_{1}}, H(\tilde{\varepsilon}, \mathcal{A}), \tilde{\varepsilon}, \mathcal{A}\right)$, mostra o seguinte resultado. 


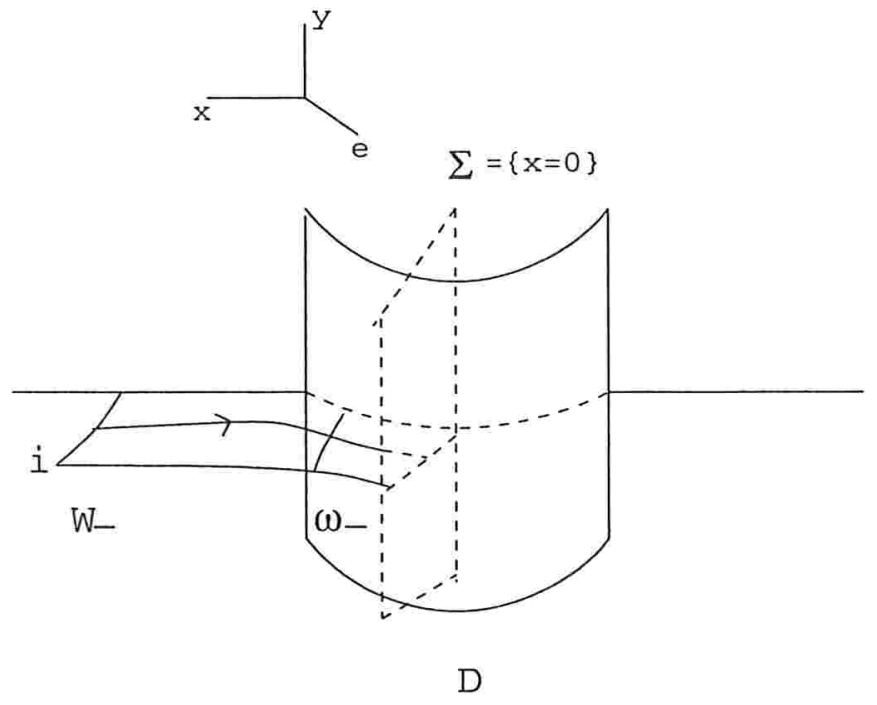

Figura 6.3: Extensão Dinâmica da Variedade Central na Carta $K_{-x_{\varepsilon}}$.

Corolário 6.2.3 Seja $\left.q \in \tilde{\xi}\right|_{\mathcal{O}^{-}}$com coordenadas

$$
q=\left\{(\tilde{x}, \tilde{y}, \tilde{\varepsilon}, \mathcal{A})=\left(\frac{-1}{e_{1}}, H(\tilde{\varepsilon}, \mathcal{A}), \tilde{\varepsilon}, \mathcal{A}\right)\right\}
$$

e seja $q^{\prime}=\phi(q, 0)=\left\{(\tilde{x}, \tilde{y}, \tilde{\varepsilon}, \mathcal{A})=\left(0, y^{\prime}, \tilde{\varepsilon}, \mathcal{A}\right)\right\}$, o único ponto onde a órbita $\{\tilde{y}=\phi(\tilde{x}, q)\}$ começando em $q$ corta $\Sigma$. Então a aplicação

$$
\begin{aligned}
Y_{-}:: & \mathcal{O}^{-} \longrightarrow \mathbf{R} \\
& (\tilde{\varepsilon}, \mathcal{A}) \longmapsto y^{\prime}=Y_{-}(\tilde{\varepsilon}, \mathcal{A})
\end{aligned}
$$

é uma função $C^{\infty}\left(\mathcal{O}^{-}\right)$.

A função $Y_{-}$será chamada de Função Transporte

- Similarmente sobre $P_{+}$, temos a extensão dinâmica $\mathcal{W}^{+}$da variedade central local $W_{+}$e o domínio maximal de extensão $\mathcal{O}^{+} \subset N_{\bar{\varepsilon}} \times V_{\mathcal{A}}$ de $W_{+}$: definimos também a correspondente função transporte $Y_{+}$: $\mathcal{O}^{+} \longmapsto \mathrm{R}$.

Geometricamente, o gráfico destas funções $Y_{-}, Y_{+}$descreve a interseç̧ão de $W_{-}, W_{+}$com a seç̧ão transversal $\Sigma$. 
Definição 6.2.4 Consideremos a função distância $\Delta \in C^{\infty}\left(\mathcal{O}^{+} \cap \mathcal{O}^{-}\right)$dada por

$$
\begin{aligned}
\Delta: & \mathcal{O}^{+} \cap \mathcal{O}^{-} \longrightarrow \mathbf{R} \\
& (\tilde{\varepsilon}, \mathcal{A}) \longmapsto \Delta(\tilde{\varepsilon}, \mathcal{A}):=Y_{+}(\tilde{\varepsilon}, \mathcal{A})-Y_{-}(\tilde{\varepsilon}, \mathcal{A})
\end{aligned}
$$

relativa às variedades centrais $W_{+}, W_{-}$.

$O$ subconjunto fechado $\mathcal{O}\left(W_{-}, W_{+}\right) \subset \mathcal{O}^{+} \cap \mathcal{O}^{-}$definido por

$$
\mathcal{O}\left(W_{-}, W_{+}\right):=\left\{(\tilde{\varepsilon}, \mathcal{A}) \in \mathcal{O}^{+} \cap \mathcal{O}^{-} \mid \Delta(\tilde{\varepsilon}, \mathcal{A})=0\right\}
$$

será chamado de região de colagem das variedades centrais $W_{+}, W_{-}$.

Agora veremos situações onde é possível obter a igualdade $\mathcal{O}\left(W_{-}, W_{+}\right)=$ $\mathcal{O}^{+} \cap \mathcal{O}^{-}$; ou seja as variedades $W_{+}, W_{-}$podem ser coladas.

Proposição 6.2.5 (Casos $(s, s),(u, s)$ )

Suponha que $\mathcal{B}_{+}<0$. Seja $W_{-}$uma variedade central dinâmica local arbitraria em $P_{-}$, definida sobre um domínio $N_{x} \times N_{e} \times V_{\mathcal{A}}$. Então, existe um subconjunto aberto (possivelmente vazio)

$$
N_{x}^{\prime} \times V_{\mathcal{A}}^{\prime} \subset N_{x} \times V_{\mathcal{A}}, \quad N_{x}^{\prime}=\left[0, x_{0}^{\prime}\right)
$$

e uma única variedade central dinâmica local $W_{+}$definida sobre o domínio aberto

$$
N_{x}^{\prime} \times N_{e}^{\prime} \times V_{\mathcal{A}}^{\prime}, \quad N_{e}^{\prime}=\left[0, e_{0}^{\prime}\right)
$$

para algum $e_{0}^{\prime}>0$, tal que $\mathcal{O}\left(W_{-}, W_{+}\right)=\mathcal{O}^{+} \cap \mathcal{O}^{-}$

DemonstraÇÃo

Consideremos a região atratora

$$
R_{+}:=\left\{(x, y, e)\left|x=0,0 \leq e<e_{+},\right| y \mid<y_{+}\right\},
$$

associada a $P_{+}$relativa a $V_{\mathcal{A}}$.

Na carta $K_{\bar{\varepsilon}}$, a intersecção $c_{-}:=W_{-} \cap D$ da variedade central dinâmica local $W_{-}$com o divisor excepcional $D=\{x=0\}$, pode ser escrita como o gráfico de uma função $C^{\infty}$

$$
c_{-}:=\operatorname{graf}\left\{\tilde{y}=y_{-}(\tilde{x}, \mathcal{A})\right\}
$$

definida para $\mathcal{A} \in V_{\mathcal{A}}$ e $\tilde{x}$ numa vizinhança de $\infty$.

Pela proposição 6.2 .2 , existe um subconjunto aberto $V_{\mathcal{A}}^{\prime}$ tal que para cada 
$\mathcal{A} \in V_{\mathcal{A}}^{\prime}$, a curva $c_{-}$estende-se para uma solução do campo $\left.\bar{X}\right|_{D}$, a qual entra na região atratora $R_{+}$. Assim, pela continuidade do fluxo de $\left.\bar{X}\right|_{D}$, existe uma pequena constante $x_{0}^{\prime}>0 \in N_{x}$, tal que restringindo $W_{-}$ao domínio

$$
\left[0, x_{0}^{\prime}\right) \times N_{e}^{\prime} \times V_{\mathcal{A}}^{\prime}, \quad N_{e}^{\prime}=\left(0, e_{0}^{\prime}\right)
$$

temos que $W_{-}$pode ser estendida a uma variedade central $\mathcal{W}_{-, \infty}$. Tal variedade corta a secção transversal $\Sigma_{e_{+}}=\left\{e=e_{+}\right\}$; assim a intersecção $\mathcal{W}_{-, \infty} \cap \Sigma_{e_{+}}=\operatorname{graf}\{i=i(x, \mathcal{A})\}$ define uma função condição inicial $i$, para o campo $\bar{X}^{+}$; logo pela proposição 6.1.4, a função $i$ define uma variedade central dinâmica local $W_{+}$em $P_{+}$. Esta variedade é tal que para cada $(\tilde{\varepsilon}, \mathcal{A}) \in \mathcal{O}^{+} \cap \mathcal{O}^{-}, \Delta(\tilde{\varepsilon}, \mathcal{A})=0$, isto mostra o resultado.

Analogamente temos o resultado para $\mathcal{B}_{-}>0$ :

Proposição 6.2.6 (Casos $(u, s),(u, u)$ )

Suponha que $\mathcal{B}_{-}>0$. Seja $W_{+}$uma variedade central dinâmica local arbitraria em $P_{+}$, definida sobre algum domínio $N_{x} \times N_{e} \times V_{\mathcal{A}}$. Então, existe um subconjunto aberto (possivelmente vazio)

$$
N_{x}^{\prime} \times N_{e}^{\prime} \times V_{\mathcal{A}}^{\prime} \subset N_{x} \times N_{e} \times V_{\mathcal{A}}, \quad N_{x}^{\prime}=\left[0, x_{0}^{\prime}\right), N_{e}^{\prime}=\left[0, e_{0}^{\prime}\right)
$$

e uma única variedade central dinâmica local $W_{-}$definida sobre o domínio aberto $N_{x}^{\prime} \times N_{e}^{\prime} \times V_{\mathcal{A}}^{\prime}$ tal que $\mathcal{O}\left(W_{-}, W_{+}\right)=\mathcal{O}^{+} \cap \mathcal{O}^{-}$.

\section{DEMONSTRAÇÃO}

Dado o conjunto $V_{\mathcal{A}}$, definimos a região atratora $R_{-}$para $P_{-}$e usando o campo $-\left.\bar{X}\right|_{D}$, mostra-se este resultado em forma análoga á anterior proposição.

\subsection{Estimativas para $\mathcal{O}\left(W_{-}, W_{+}\right)$}

Aqui usando as estimativas assintóticas da família Riccati $R_{A, B}(\mathcal{Q})$ sobre a carta $K_{\bar{\varepsilon}}$, aparece a existência de regiões contidas em $\mathcal{O}\left(W_{-}, W_{+}\right)$, sobre as quais tais variedades colam-se. Mais precisamente temos os seguintes resultados.

Lema 6.3.1 $\left(\mathcal{O}\left(W_{-}, W_{+}\right)\right.$, sobre os casos $\left.(s, s),(u, s)\right)$

Assumamos que $\mathcal{B}_{+}<0$, e seja $W_{-}$uma variedade central dinâmica local 
em $P_{-}$, definida sobre um dominio $N_{x} \times N_{e} \times V_{\mathcal{A}}$, com $\mathcal{A}=0 \in V_{\mathcal{A}}$. Suponha que a curva

$$
y_{-}^{(0,0)}:=\left.W_{-} \cap D\right|_{\mathcal{A}=0}
$$

é uma solução da familia Riccati $R_{A, B}(\mathcal{Q})$ que não escapa ao infinito em tempo finito. Então existe uma variedade central $W_{+}$definida sobre algum dominio $N_{x}^{\prime} \times N_{e}^{\prime} \times V_{\mathcal{A}}^{\prime}$, tal que $\mathcal{O}\left(W_{-}, W_{+}\right)$contém uma vizinhança aberta $U$ da origem $(\tilde{\varepsilon}, \mathcal{A})=(0,0)$ em $\left(\mathbf{R}^{+} \times \mathbf{R}^{n}, 0\right)$.

\section{DEMONSTRAÇÃo}

Da proposição 6.2 .5 , segue a existência de $W_{+}$para o qual $\mathcal{O}\left(W_{-}, W_{+}\right)=$ $\mathcal{O}^{-} \cap \mathcal{O}^{+}$, e dos lemas 5.4.1 e 5.4.2 temos que $W_{-} \cap D$ corta a secção $\Sigma_{e_{+}}$, para parâmetros $\left(A, B, \mathcal{A}_{r}\right)$ numa vizinhança suficientemente pequena de $\left(A, B, \mathcal{A}_{r}\right)=(0,0,0)$. O resultado segue se escolhemos $\tilde{\varepsilon}>0$ suficientemente pequeno.

Analogamente segue o resultado para $\mathcal{B}_{-}>0$.

Lema 6.3.2 $\left(\mathcal{O}\left(W_{-}, W_{+}\right)\right.$, sobre os casos $\left.(u, s),(u, u)\right)$

Assumamos que $\mathcal{B}_{-}>0$, e seja $W_{+}$uma variedade central dinâmica local em $P_{+}$, definida sobre um dominio $N_{x} \times N_{e} \times V_{\mathcal{A}}$, com $\mathcal{A}=0 \in V_{\mathcal{A}}$. Suponha que a curva

$$
y_{+}^{(0,0)}:=\left.W_{+} \cap D\right|_{\mathcal{A}=0}
$$

é uma solução da familia Riccati $R_{A, B}(\mathcal{Q})$ que não escapa ao infinito em tempo finito. Então existe uma variedade central $W_{-}$definida sobre algum dominio $N_{x}^{\prime} \times N_{e}^{\prime} \times V_{A}^{\prime}$, tal que $\mathcal{O}\left(W_{-}, W_{+}\right)$contém uma vizinhança aberta $U$ da origem $(\tilde{\varepsilon}, \mathcal{A})=(0,0)$ em $\left(\mathbf{R}^{+} \times \mathbf{R}^{n}, 0\right)$.

DEMONSTRAÇÃO

Similarmente, para $\tilde{\varepsilon}$ suficientemente pequeno o resultado segue da proposição 6.2 .6 e os lemas 5.4 .1 e 5.4 .2 .

Observação 6.3.3 - No caso linear, as soluçòes da famılia $R_{A, B}(0)$ não escapam ao infinito em tempo finito; logo para um conjunto aberto 
$V_{A, B}^{\prime} \subset \mathbf{R}^{3 p}$ arbitrário com fecho contido em $V_{\mathcal{A}} \cap\left\{\mathcal{A}_{r}=0\right\}$, existem dominios

$$
N_{x}^{\prime}, N_{e}^{\prime} \subset \mathbf{R}^{+}, V_{\mathcal{A}_{r}}^{\prime} \in\left(\mathbf{R}^{n-3 p}, 0\right)
$$

tal que as variedades centrais $W_{-}, W_{+}$definidas sobre $N_{x}^{\prime} \times N_{e}^{\prime} \times\left(V_{A, B}^{\prime} \times\right.$ $V_{\mathcal{A}_{r}}^{\prime}$ ) colam-se em uma região da forma

$$
\mathcal{O}\left(W_{-}, W_{+}\right)=N_{\tilde{\varepsilon}} \times\left(V_{A, B}^{\prime} \times V_{\mathcal{A}_{r}}^{\prime}\right)
$$

Onde $N_{\bar{\varepsilon}}=\left[0, \tilde{\varepsilon}_{0}\right) \subset \mathbf{R}^{+}$.

- Podemos representar com a notação $W_{-} \longrightarrow W_{+}$, o fato que a variedade central $W_{-}$em $P_{-}$, induz a variedade central $W_{+}$em $P_{+}$; assim dos lemas 6.3 .1 e 6.3.2 temos

$$
\begin{aligned}
& \text { - No caso }(s, s) ; W_{-} \longrightarrow W_{+}, \\
& \text {- No caso }(u, s) ; W_{-} \longrightarrow W_{+} \text {e } W_{+} \longrightarrow W_{-}, \\
& \text {- No caso }(u, u) ; W_{+} \longrightarrow W_{-} .
\end{aligned}
$$

Agora veremos o caso especial de transição $(s, u)$, onde a região de colagem $\mathcal{O}\left(W_{-}, W_{+}\right)$é um conjunto fechado de codimensão um, definido pelo gráfico de uma função $C^{\infty}$.

Lema 6.3.4 Sejam $W_{-}$e $W_{+}$variedades centrais locais em $P_{-}$e $P_{+}$respectivamente, definidas sobre um comum domínio aberto $N_{x} \times N_{e} \times V_{\mathcal{A}}$ com $\mathcal{A}=0 \in V_{\mathcal{A}}$. Então o domínio maximal de extensão comum destas variedades centrais

$$
\mathcal{O}^{-} \cap \mathcal{O}^{+} \subset N_{\tilde{\varepsilon}} \times V_{\mathcal{A}}
$$

onde $N_{\tilde{\varepsilon}}$ é como em (6.3), contém uma vizinhança aberta $U$ da origem $(\tilde{\varepsilon}, \mathcal{A})=(0,0)$.

\section{DEMONSTRAÇÃo}

Fazendo $K=0$ no Lema 5.4 .1 e para $\left(A, B, \mathcal{A}_{r}\right)$ em algum subconjunto aberto $N \subset V_{\mathcal{A}}$, segue que as soluções $y_{-}=W_{-} \cap D, y_{+}=W_{+} \cap D$ da família Riccati $R_{A, B}(\mathcal{Q})$ cortam a secção transversal $\Sigma=\{x=0\}$; logo as variedades centrais $W_{-}, W_{+}$também cortam transversalmente a secção $\Sigma$, para $\tilde{\varepsilon}$ suficientemente pequeno. 
Proposição 6.3.5 $\left(\mathcal{O}\left(W_{-}, W_{+}\right)\right.$sobre o caso $\left.(s, u)\right)$

Usando as notações do lema 6.3.4, fixemos um índice par arbitrário $s \in$ $\{0,1, \ldots, 2 p-1\}$. Então existe uma vizinhança da origem $U_{s} \subset U$ e uma função $C^{\infty} a_{s}: \hat{U}_{s} \longrightarrow \mathbf{R}, \quad a_{s}(0)=0$ definida sobre o dominio $\hat{U}_{s}:=$ $U_{s} \cap\left\{A_{s}=0\right\}$, tal que a restrição da região $\mathcal{O}\left(W_{-}, W_{+}\right)$a $U_{s}$ é dada pelo gráfico de $a_{s}$

$$
\mathcal{O}\left(W_{-}, W_{+}\right) \cap U_{s}=\operatorname{graf}\left\{A_{s}=a_{s}\left(\tilde{\varepsilon}, \hat{A}, B, \mathcal{A}_{r}\right)\right\},
$$

onde $\hat{A}=\left(A_{0}, \ldots, A_{s-1}, A_{s+1}, \ldots, A_{2 p-1}\right)$.

\section{DEmonstraÇÃo}

Como a função distância $\Delta: \mathcal{O}^{-} \cap \mathcal{O}^{+} \longrightarrow \mathbf{R}$ está definida sobre alguma vizinhança aberta $U \subset \mathcal{O}^{-} \cap \mathcal{O}^{+}$da origem $(\tilde{\varepsilon}, \mathcal{A})=(0,0)$, podemos considerar a expansão $\Delta$ no ponto $(0, A, B, 0)$

$$
\Delta\left(\tilde{\varepsilon}, A, B, \mathcal{A}_{r}\right)=\delta(A, B)+O\left(\tilde{\varepsilon}, \mathcal{A}_{r}\right)
$$

Onde $\delta$ é como no Lema 5.4.3.

Logo o resultado segue do teorema da função implícita pois

$$
\left.\frac{\partial \Delta}{\partial A_{s}}\right|_{D_{0}}=\frac{\partial \delta}{\partial A_{s}} \neq 0
$$

sobre o divisor excepcional $D_{0}=\left\{\tilde{\varepsilon}=\mathcal{A}_{r}=0\right\}$. 


\section{Capítulo 7}

\section{Superfícies Canard}

Com as hipóteses e notações do capítulo 2, consideremos a família de perturbações singulares

$$
X=\varepsilon \frac{\partial}{\partial x}+F(x, y, \varepsilon, \mathcal{A}) \frac{\partial}{\partial y}
$$

definida nas coordenadas $(x, y, \varepsilon, \mathcal{A})$ sobre o subconjunto $U=U_{x} \times U_{y} \times$ $U_{\varepsilon} \times U_{\mathcal{A}}$, onde $U_{x}$ é um conjunto aberto conexo, $\Gamma=\{y=\varepsilon=\mathcal{A}=0\}$ a curva de singularidades para $X$, e $x=0$ é uma singularidade degenerada satisfazendo à Hipótese de Transversalidade.

Do capítulo 3 , temos que para $x_{0} \neq 0 \in \Gamma$ e qualquer subconjunto aberto $V \subset U_{\varepsilon, \mathcal{A}}$ podemos considerar uma função condição inicial

$$
i \in C_{\text {flat }}^{\infty}\left(V,\{\varepsilon=0\}, \widehat{W}_{x_{0}}\right)
$$

para $X$ em $x_{0}$ de maneira que para qualquer ponto $x_{1} \neq x_{0}$, tal que

$$
\left\{\begin{array}{l}
0>x_{1}>x_{0}, \text { se } \quad \mathcal{B}_{-}<0 \\
0<x_{1}<x_{0}, \text { se } \quad \mathcal{B}_{+}>0
\end{array}\right.
$$

existe uma única variedade central dinâmica

$$
W\left(\Gamma_{x_{0}, x_{1}}\right)=\operatorname{graf}\{y=w(x, \varepsilon, \mathcal{A})\},
$$

definida sobre o domínio $\Gamma_{x_{0} . x_{1}} \times \mathcal{O}_{\Gamma_{x_{0} . x_{1}}}$, (onde $\mathcal{O}_{\Gamma_{x_{0}, x_{1}}} \subset V$, é um aberto e $\left.\Gamma_{x_{0}, x_{1}}=\left[x_{0}, x_{1}\right]\right)$ e tal que $w\left(x_{0}, \varepsilon, \mathcal{A}\right)=i(\varepsilon, \mathcal{A})$.

Com a finalidade de mostrar a existência das Superfícies Canard, vamos considerar alguns resultados de extensão pela aplicação Blowing-up $\Phi$ definida no capítulo 2 , onde as variedades centrais dinâmiças $W\left(\Gamma_{x_{0} . x_{1}}\right)$ serão estendidas as variedades centrais dinâmica $W_{-}, W_{+}$respectivamente. 


\subsection{Blowing-up e Blowing-down de $\mathcal{O}\left(W_{-}, W_{+}\right)$}

Sobre a carta $K_{ \pm \bar{x}_{\varepsilon}}$, consideremos o campo $\bar{X}$ definido pela aplicação Blowing-up principal $\Phi: \bar{U} \longrightarrow U$.

Nas variáveis adaptadas $(\varepsilon, a, b, \mathcal{A})$ sobre a carta $K_{\bar{\varepsilon}}$, restringindo o Blowingup, obtemos a aplicação polinomial

$$
\varphi:\left(\tilde{\varepsilon}, A_{i}, B_{j}, \mathcal{A}_{r}\right) \longrightarrow\left(\varepsilon, a_{i}, b_{j}, \mathcal{A}_{r}\right)=\left(\tilde{\varepsilon}^{p+1}, \tilde{\varepsilon}^{2 p-i} A_{i}, \tilde{\varepsilon}^{p-j} B_{j}, \mathcal{A}_{r}\right),
$$

onde $0 \leq i \leq 2 p-1, \quad 0 \leq j \leq p-1$.

Similarmente sobre a carta $K_{ \pm \bar{x}_{\varepsilon}}$, para cada ponto fixado $\hat{x}=\hat{x}_{0}$ o blowingup restrito às variáveis $(\varepsilon, a, b, \mathcal{A})$, é a aplicação polinomial

$$
\phi_{\hat{x}_{0}}:\left(e, A_{i}, B_{j}, \mathcal{A}_{r}\right) \longrightarrow\left(\hat{x}_{0}^{p+1} e^{p+1}, \hat{x}_{0}^{2 p-i} e^{2 p-i} A_{i}, \hat{x}_{0}^{p-j} e^{p-j} B_{j}, \mathcal{A}_{r}\right)
$$

Definindo a constante positiva $\hat{x}_{0}:=\left|x_{0}\right|, \quad V_{\hat{\mathcal{A}}}:=V_{A, B} \times V_{\mathcal{A}_{r}}$, onde $V_{A, B} \subset \mathbb{R}^{3 p}$ tem fecho compacto e $V_{\mathcal{A}_{r}} \in\left(\mathbb{R}^{n-3 p}, 0\right)$, obtemos os seguintes resultados.

\section{Lema 7.1.1 Com as notações acima}

(a) Suponha que $\mathcal{B}_{-}:=(-1)^{p} \mathcal{B}_{0}<0, x_{0}<0$ e que existe um subconjunto aberto $V_{e}=\left(0, e_{0}\right), e_{0}>0$ tal que $V_{e} \times V_{\hat{\mathcal{A}}} \subset \phi_{\hat{x}_{0}}^{-1}(V)$, com $\hat{x}_{0} \in V_{x}$. Então a aplicação

$$
I(e, \hat{\mathcal{A}}):=\left(\frac{1}{\hat{x}_{0}^{p}}\right) i \circ \phi_{\hat{x}_{0}}(e, \hat{\mathcal{A}})
$$

é uma função condição inicial para $\bar{X}$ em $P_{-}$, sobre o domínio $V_{e} \times V_{\hat{\mathcal{A}}}$.

(b) Reciprocamente, se $W_{-}=\operatorname{graf}\left\{y=w_{-}(\hat{x}, e, \hat{\mathcal{A}})\right\}$ é uma variedade central sobre $P_{-}$definida sobre o domínio $N_{\hat{x}} \times N_{e} \times V_{\hat{A}}$, tal que $I(e, \hat{\mathcal{A}}):=w_{-}\left(\hat{x}_{0}, e, \hat{\mathcal{A}}\right) ;$ a aplicação

$$
i(\varepsilon, a, b):=\left(\hat{x}_{0}^{p}\right) I \circ \phi_{\hat{x}_{0}}^{-1}(\varepsilon, a, b)
$$

é a função condição inicial para $X$ em $x_{0}=-\hat{x}_{0}$, com domínio de definição $\phi_{\hat{x}_{0}}\left(V_{e} \times V_{\hat{\mathcal{A}}}\right)=V$.

DEMONSTRAÇÃo

Consideremos a expansão formal em serie $\widehat{W}_{-, \hat{x}_{0}}$ da variedade central $W_{-}$restrita a $\hat{x}_{0}$, para o campo $\bar{X}$ em $P_{-}$. Por hipótese temos que

$$
i-\widehat{W}_{x_{0}} \in C_{f l a t}^{\infty}(V,\{\varepsilon=0\}),
$$


onde $\widehat{W}_{x_{0}}$ é a série formal da variedade central $W\left(\Gamma_{x_{0}, x_{1}}\right)$ para o campo $X$ em $x_{0}=-\hat{x}_{0}$. Logo pela proposição 2.1 .13 obtemos

$$
I-\widehat{W}_{-, \hat{x}_{0}} \in C_{\text {flat }}^{\infty}\left(V_{e} \times V_{\hat{\mathcal{A}}},\{e=0\}\right)
$$

e isto mostra o item $(a)$.

O item (b), é análogo

O resultado análogo para $\mathcal{B}_{+}$é o seguinte

Lema 7.1.2 Aqui

(a) Suponha que $\mathcal{B}_{+}>0, x_{0}>0$. Então a aplicação

$$
I(e, \hat{\mathcal{A}}):=\left(\frac{1}{\hat{x}_{0}^{p}}\right) i \circ \phi_{\hat{x}_{0}}(e, \hat{\mathcal{A}})
$$

é uma função condição inicial para $\bar{X}$ em $P_{+}$, sobre o domínio $V_{e} \times$ $V_{\hat{\mathcal{A}}} \subset \phi_{\hat{x}_{0}}^{-1}(V)$ com fecho compacto.

(b) Reciprocamente, se $W_{+}=$graf $\left\{y=w_{+}(\hat{x}, e, \hat{\mathcal{A}})\right\}$ é uma variedade central em $P_{+}$definida sobre o dominio $N_{\hat{x}} \times N_{e} \times V_{\hat{\mathcal{A}}}$, tal que $I(e, \hat{\mathcal{A}}):=$ $w_{+}\left(\hat{x}_{0}, e, \hat{\mathcal{A}}\right) ;$ a aplicação

$$
i(\varepsilon, a, b):=\left(\hat{x}_{0}^{p}\right) I \circ \phi_{\hat{x}_{0}}^{-1}(\varepsilon, a, b)
$$

é a função condição inicial para $X$ em $x_{0}=\hat{x}_{0}$, com domínio de definição $\phi_{\grave{x}_{0}}\left(V_{e} \times V_{\hat{\mathcal{A}}}\right)=V$.

\section{DEMONSTRAÇÃO}

Similar ao lema anterior.

Denotemos por $\Gamma_{-}, \Gamma_{+} \subset \Gamma$, os intervalos abertos definidos por

$$
\Gamma_{-}:=\left\{x \in U_{x} \mid x<0\right\}, \quad \Gamma_{+}:=\left\{x \in U_{x} \mid x>0\right\}
$$

e $\mathcal{O} \subset\{(\varepsilon, \hat{\mathcal{A}}) \mid \varepsilon>0\}$, um domínio em $V$. Dos lemas anteriores, segue os resultados de extensão blowing-up, blowing-down. 
Proposição 7.1.3 Suponha que $\mathcal{B}_{-}<0$, (resp. $\left.\mathcal{B}_{+}>0\right)$ e seja $W(\widetilde{\Gamma} \times \mathcal{O})$ qualquer variedade central com dominio $\widetilde{\Gamma} \times \mathcal{O}$, onde

$$
\tilde{\Gamma} \subset \Gamma_{-},\left(\operatorname{resp} . \quad \widetilde{\Gamma} \subset \Gamma_{+}\right)
$$

é um subintervalo compacto conexo. Então, para qualquer constante positiva suficientemente pequena $\hat{x}_{0}=\left|x_{0}\right| \in U_{x}$, e qualquer subconjunto aberto com fecho compacto $V_{e} \times V_{\mathcal{A}}=\left(0, e_{0}\right) \times\left(V_{A, B} \times V_{\mathcal{A}_{r}}\right) \subset \phi_{\hat{x}_{0}}^{-1}(V)$, existe uma pequena constante $0<e_{1}<e_{0}$ tal que $W(\widetilde{\Gamma} \times \mathcal{O})$ estende-se unicamente como uma variedade central $W_{-}$em $P_{-}$, (resp. $W_{+}$em $\left.P_{+}\right)$, definida sobre o domínio

$$
N_{\hat{x}} \times N_{e} \times V_{\hat{\mathcal{A}}}, \quad N_{e}:=\left(0, e_{1}\right), \quad N_{\hat{x}}:=\left[0, \hat{x}_{0}\right] .
$$

\section{DEmonstração}

Dos lemas 7.1.1(a) e 7.1.2(a), temos que a função $\phi_{\hat{x}_{0}}^{*}(i)$ cujo gráfico é dado por

$$
W(\widetilde{\Gamma} \times \mathcal{O}) \cap\left\{x=-\hat{x}_{0}\right\},\left(\text { resp. } W(\widetilde{\Gamma} \times \mathcal{O}) \cap\left\{x=\hat{x}_{0}\right\}\right)
$$

é uma função condição inicial para $\bar{X}$ em $P_{-}$, (resp. $\bar{X}$ em $P_{+}$). Logo da proposição 6.1 .3 obtemos a extensão desejada.

A extensão recíproca é como segue

Proposição 7.1.4 Seja $W_{-}$qualquer variedade central em $P_{-}$, (resp. $W_{+}$ em $\left.P_{+}\right)$definida sobre o dominio $N_{\hat{x}} \times N_{e} \times V_{\hat{\mathcal{A}}}$. Suponha que $\mathcal{B}_{-}>0$ (resp. $\left.\mathcal{B}_{+}<0\right)$. Então, para qualquer constante positiva $\hat{x}_{0} \in N_{\hat{x}}$ e qualquer subintervalo compacto

$$
\tilde{\Gamma} \subset \Gamma_{-},\left(\text {resp. } \quad \tilde{\Gamma} \subset \Gamma_{+}\right)
$$

existe uma vizinhança $N=N(\tilde{\Gamma}) \subset U_{\varepsilon, \mathcal{A}}$ da origem $(\varepsilon, \mathcal{A})=(0,0)$ tal que $W_{-}$(resp. $W_{+}$) estende-se unicamente como uma variedade central $W(\widetilde{\Gamma} \times \mathcal{O})$, onde

$$
\mathcal{O}:=\phi_{\hat{x}_{0}}\left(N_{e} \times V_{\hat{\mathcal{A}}}\right) \cap N
$$

DEMONSTRAÇÃO

Dos lemas 7.1.1(b) e 7.1.2(b), temos que a função $\left(\phi_{\hat{x}_{0}}^{*}\right)^{-1}(I)$ com gráfico

$$
W_{-} \cap\left\{x=\hat{x}_{0}\right\},\left(\text { resp. } W_{+} \cap\left\{x=\hat{x}_{0}\right\}\right)
$$


é uma função condição inicial para $X$ em $\Gamma_{-}$,(resp. $X$ em $\Gamma_{+}$). Logo por continuidade do blowing-up, da hipótese segue que $\mathcal{B}_{-\hat{x}_{0}}>0$ para qualquer $x_{0}=-\hat{x}_{0}<0 \in \Gamma_{-}\left(\right.$resp. $\mathcal{B}_{\hat{x}_{0}}<0, \forall x_{0}=\hat{x}_{0}>0 \in \Gamma_{+}$), assim o resultado segue aplicando proposição 3.3.3.

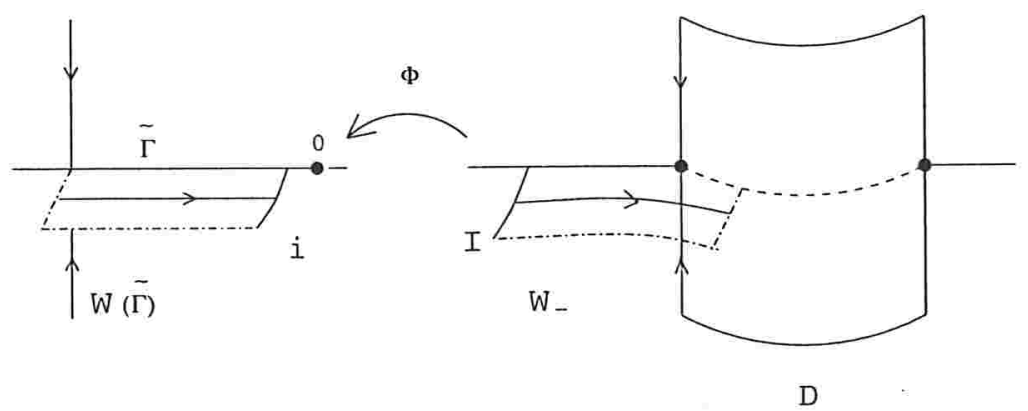

Figura 7.1: Blowing-down para o caso $\mathcal{B}_{-}<0$.

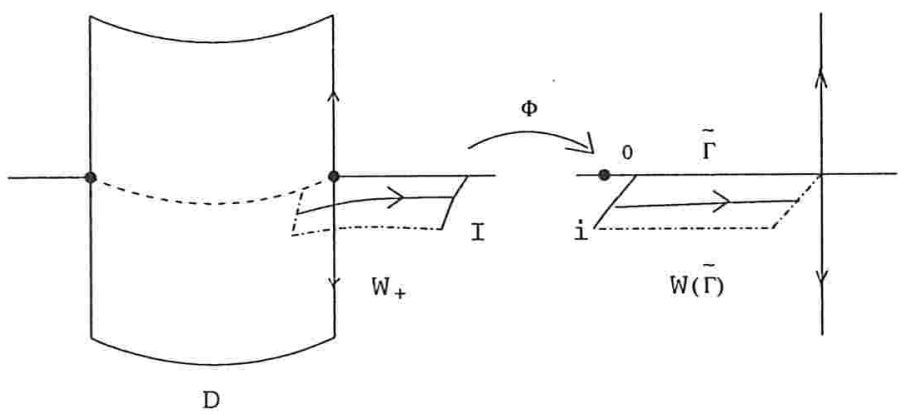

Figura 7.2: Blowing-down para o caso $\mathcal{B}_{+}>0$.

Observação 7.1.5 Notemos que

- Os resultados de extensão acima (ver figuras 7.1 e 7.2), mostram os casos especiais $(s, u),(u, s)$ respectivamente, que esquematicamente podemos expressar como

$$
\begin{aligned}
& W\left(\Gamma_{-}\right) \longrightarrow^{\mathcal{B}_{-}<0} W_{-}, \quad W_{+} \longleftarrow^{\mathcal{B}_{+}>0} W\left(\Gamma_{+}\right) ; \\
& W\left(\Gamma_{-}\right){ }^{\mathcal{B}_{-}>0} W_{-}, \quad W_{+} \longrightarrow^{\mathcal{B}_{+}<0} W\left(\Gamma_{+}\right) .
\end{aligned}
$$


- Os casos $(s, s),(u, u)$ são análogos. Com a notação introduzida no capítulo anterior temos o esquema

$$
\begin{aligned}
& W\left(\Gamma_{-}\right) \longrightarrow^{\mathcal{B}_{-}<0} W_{-} \longrightarrow W_{+} \longrightarrow^{\mathcal{B}_{+}<0} W\left(\Gamma_{+}\right) ; \\
& W\left(\Gamma_{-}\right){ }^{\mathcal{B}_{-}>0} W_{-} \longleftarrow W_{+} \longleftarrow^{\mathcal{B}_{+}>0} W\left(\Gamma_{+}\right) .
\end{aligned}
$$

Agora estamos prontos para abordar os resultados principais deste trabalho.

\subsection{Existência de Superfícies Canard}

Seja $X$ um campo singularmente perturbado definido sobre o aberto $U=U_{x} \times U_{y} \times U_{\varepsilon} \times U_{\mathcal{A}}$, descrito no capítulo 2 , seção 2.3 tal que nas coordenadas $(x, y, \varepsilon, \mathcal{A})$ temos que:

1. $X$ satisfaz a Hipótese de Transversalidade na única singularidade degenerada

$$
x=0 \in \Gamma=\{y=\varepsilon=\mathcal{A}=0\} \approx U_{x}
$$

com multiplicidade $p=\mu(X)$.

2. $(\varepsilon, \mathcal{A})=\left(\varepsilon, a, b, \mathcal{A}_{r}\right) \in U_{\varepsilon} \times U_{\mathcal{A}}$ são os parâmetros adaptados para $X$.

Vamos considerar constantes positivas $S, R>0$ tal que

$$
\{0<\varepsilon<R,\|\mathcal{A}\|<S\} \subset V \subset U_{\varepsilon} \times U_{\mathcal{A}} \cap\{\varepsilon=0\},
$$

e também um subconjunto compacto conexo $\widetilde{\Gamma} \subset \Gamma$ da forma

$$
\widetilde{\Gamma}=\left[x_{0}, x_{1}\right], \quad x_{0}<0<x_{1} .
$$

Vamos escrever mais precisamente no espaço de parâmetros $U_{\tilde{\varepsilon}, \mathcal{A}}$ as regiões $\mathcal{O}_{\widetilde{\Gamma}} \subset V$ para as quais existem as Superfícies Canard $\{y=w(x, \varepsilon, \mathcal{A})\}$, em cada caso de transição $(s, s),(u, u),(u, s),(s, u)$ para $x=0$. O par $\left(\mathcal{O}_{\widetilde{\Gamma}}, w\right)$, denotará tal superfície.

\subsection{1 $\left(\mathcal{O}_{\widetilde{\Gamma}}, w\right)$ nos casos $(s, s),(u, u)$}

No caso $(s, s)$ consideramos o ponto base $x=x_{0} \in \widetilde{\Gamma}$, a partir do qual será definida a variedade central formal

$$
\widehat{W}(x, \varepsilon, \mathcal{A})=\sum_{i=0}^{\infty} w_{i}(x, \mathcal{A}) \varepsilon^{i} .
$$

O caso $(u, u)$ pode ser tratado analogamente, considerando o campo $-X$ sobre o ponto base $x=x_{1} \in \widetilde{\Gamma}$ 
Teorema 7.2.1 Suponha que $X$ está no caso $(s, s)$ e seja $i \in C_{\text {flat }}^{\infty}(V,\{\varepsilon=$ $\left.0\}, \widehat{W}_{x_{0}}\right)$ uma função condição inicial para $X$ em $x=x_{0}$. Então existem uma coleção de constantes estritamente positivas $\left\{m, r, k_{i}, l_{j}\right\}$ definindo a Região Canard $\mathcal{O}_{\widetilde{\Gamma}}$ como um conjunto aberto da forma

$$
\mathcal{O}_{\widetilde{\Gamma}}= \begin{cases}0<\varepsilon<r & \\ \left|a_{i}\right|<k_{i}|\varepsilon|^{\frac{2 p-i}{p+1}} & 0 \leq i \leq 2 p-1 \\ \left|b_{j}\right|<l_{j}|\varepsilon|^{\frac{p-j}{p+1}} & 0 \leq j \leq p-1 \\ \left|\mathcal{A}_{r}\right|<m & \end{cases}
$$

e uma única função $C^{\infty}$

$$
\begin{array}{ll}
w: \quad & \widetilde{\Gamma} \times \mathcal{O}_{\widetilde{\Gamma}} \longrightarrow \mathbf{R} \\
& (x,(\varepsilon, \mathcal{A})) \longmapsto y=w(x, \varepsilon, \mathcal{A})
\end{array}
$$

satisfazendo o seguinte

(i) $w\left(x_{0}, \varepsilon, \mathcal{A}\right)=i(\varepsilon, \mathcal{A}), \forall(\varepsilon, \mathcal{A}) \in \mathcal{O}_{\widetilde{\Gamma}}$;

(ii) $W(\widetilde{\Gamma}):=\operatorname{graf}\{y=w(x, \varepsilon, \mathcal{A})\}$ é uma variedade invariante por $X$;

(iii) - w tem extensão contínua ao fecho de $\widetilde{\Gamma} \times \mathcal{O}_{\widetilde{\Gamma}}$, tal que $w(x, 0,0) \equiv$ 0 ;

- $w$ tem uma extensão blowing-up $C^{\infty}$ em $\{x=\varepsilon=0\}$.

DEMONSTRAÇÃO

Da última observação, o resultado é mostrado pelo esquema

$$
W\left(\Gamma_{-}\right) \longrightarrow^{\mathcal{B}_{-}<0} W_{-} \longrightarrow W_{+} \longrightarrow^{\mathcal{B}_{+}<0} W\left(\Gamma_{+}\right),
$$

onde o par $\left(\mathcal{O}_{\widetilde{\Gamma}}, w\right)$ é construído como segue.

Passo 1 Para cada $x_{0}^{\prime} \in U_{x}$, com $x_{0}<x_{0}^{\prime}<0$, definamos o subintervalo compacto $\Gamma_{0}:=\left[x_{0}, x_{0}^{\prime}\right]$. Pela Proposição 3.3 .3 , existem uma vizinhança $N_{0} \subset U_{\varepsilon, \mathcal{A}}$ da origem $(\varepsilon, \mathcal{A})=(0,0)$ e uma função $C^{\infty}$

$$
\begin{aligned}
w_{0}: \Gamma_{0} \times \mathcal{O}_{0} \longrightarrow \mathbf{R} \\
\\
(x, \Xi, \mathcal{A}) \longmapsto y=w_{0}(x, \Xi, \mathcal{A}),
\end{aligned}
$$

onde $\mathcal{O}_{0}:=V \cap N_{0}$, tal que

(i) $w_{0}\left(x_{0}, \varepsilon, \mathcal{A}\right)=i(\varepsilon, \mathcal{A}), \forall(\varepsilon, \mathcal{A}) \in \mathcal{O}_{0}$; 
(ii) $W\left(\Gamma_{0}\right):=\operatorname{graf}\left\{y=w_{0}(x, \varepsilon, \mathcal{A})\right\}$ é uma variedade invariante por $X$

(iii) $w_{0} \in C_{\text {flat }}^{\infty}\left(\Gamma_{0} \times \mathcal{O}_{0},\{\varepsilon=0\}, \widehat{W}\right)$.

Considerando $\hat{x}_{0}^{\prime}=\left|x_{0}^{\prime}\right|$ suficientemente pequeno, de (7.3) existe sobre a carta $K_{-\bar{x}_{\varepsilon}}$ uma vizinhança aberta da origem $(e, \hat{\mathcal{A}})=(0,0)$ contida no conjunto $\phi_{\hat{x}_{0}^{\prime}}^{-1}\left(\mathcal{O}_{0}\right)$.

Como $\mathcal{B}_{-}<0$, segue da proposição 7.1 .3 que a variedade $W\left(\Gamma_{0}\right)$ é estendida unicamente como uma variedade central

$$
W_{-}:=\operatorname{graf}\left\{\hat{y}=\hat{w}_{-}(\hat{x}, e, \hat{\mathcal{A}})\right\}
$$

Em $P_{-}$, definida sobre o domínio $N_{\hat{x}} \times N_{e} \times V_{\hat{\mathcal{A}}}$, onde

$$
N_{\hat{x}}:=\left[0, \hat{x}_{0}^{\prime}\right], \quad N_{e}:=\left(0, e_{0}\right), \quad V_{\hat{\mathcal{A}}} \in\left(\mathbf{R}^{n}, 0\right) .
$$

Passo 2 Como $\mathcal{B}_{+}<0$, o Lema 6.3.1 implica que $W_{-}$induz uma única variedade central em $P_{+}$

$$
W_{+}:=\operatorname{graf}\left\{\hat{y}=\hat{w}_{+}(\hat{x}, e, \hat{\mathcal{A}})\right\}
$$

definida sobre o domínio $N_{x}^{\prime} \times N_{e}^{\prime} \times V_{\hat{\mathcal{A}}}^{\prime}$, onde

$$
N_{x}^{\prime}:=\left[0, \hat{x}_{1}^{\prime}\right], \quad N_{e}^{\prime}=\left(0, e_{1}\right), \quad V_{\hat{\mathcal{A}}}^{\prime} \subset V_{\hat{\mathcal{A}}} .
$$

A região de colagem $\mathcal{O}\left(W_{-}, W_{+}\right)$contém uma vizinhança aberta da origem $(\tilde{\varepsilon}, \tilde{\mathcal{A}})=(0,0)$ em $U_{\tilde{\varepsilon}, \tilde{\mathcal{A}}}$; daqui podemos escolher as constantes $r, m, k_{i}, l_{j}$ estritamente positivas tal que o conjunto

$$
\Omega= \begin{cases}0<\tilde{\varepsilon}<r^{\frac{1}{p+1}} & \\ \left|A_{i}\right|<k_{i} & 0 \leq i \leq 2 p-1 \\ \left|B_{j}\right|<l_{j} & 0 \leq j \leq p-1 \\ \left|\mathcal{A}_{r}\right|<m & \end{cases}
$$

é estritamente contido em $\mathcal{O}\left(W_{-}, W_{+}\right)$. Além disso vale

$$
W_{-} \equiv W_{+}=\operatorname{graf}\{\tilde{y}=\tilde{w}(\tilde{x}, \tilde{\Xi}, \tilde{\mathcal{A}})\}
$$

para alguma função $\tilde{w} \in C^{\infty}\left(\mathbf{R} \times \mathcal{O}\left(W_{-}, W_{+}\right)\right)$. 
Passo 3 Da Proposição 7.1.4, existe uma vizinhança $N_{1} \subset U_{\varepsilon, \mathcal{A}}$ da origem $(\varepsilon, \mathcal{A})=(0,0)$ e uma única função $C^{\infty}$ definida por

$$
\begin{aligned}
w_{1}: \Gamma_{1} \times \mathcal{O}_{1} & \longrightarrow \mathbb{R} \\
(x, \varepsilon, \mathcal{A}) & \longmapsto y=w_{1}(x, \varepsilon, \mathcal{A}) ;
\end{aligned}
$$

onde $\Gamma_{1}:=\left[x_{1}^{\prime}, x_{1}\right], \quad \mathcal{O}_{1}=N_{1} \cap \phi_{x_{1}^{\prime}}\left(N_{e}^{\prime} \times V_{\hat{\mathcal{A}}}^{\prime}\right)$ tal que $W_{+}$estende-se unicamente como a variedade central

$$
W\left(\Gamma_{1}\right):=\operatorname{graf}\left\{y=w_{1}(x, \varepsilon, \mathcal{A})\right\} .
$$

Verificando as propriedades análogas de $W\left(\Gamma_{0}\right)$

(i) $w_{1}\left(x_{1}, \varepsilon, \mathcal{A}\right)=i(\varepsilon, \mathcal{A}), \forall(\varepsilon, \mathcal{A}) \in \mathcal{O}_{1}$,

(ii) $W\left(\Gamma_{1}\right)$ é uma variedade invariante por $X$,

(iii) $w_{1} \in C_{\text {flat }}^{\infty}\left(\Gamma_{1} \times \mathcal{O}_{1},\{\varepsilon=0\}, \widehat{W}\right)$.

Como na carta $K_{\bar{\varepsilon}}$, vale $\varepsilon=\tilde{\varepsilon}^{p+1}$, de um cálculo direto obtemos a Região Canard $\mathcal{O}_{\tilde{\Gamma}}$ definida por

$$
\mathcal{O}_{\tilde{\Gamma}}:=\varphi(\Omega)
$$

sendo $\varphi$ a função polinomial dada em (7.1); e ajustando as constantes $r, m, k_{i}, l_{j}$ podemos conseguir a inclusão

$$
\mathcal{O}_{\tilde{\Gamma}} \subset \mathcal{O}_{0} \cap \mathcal{O}_{1}
$$

Neste caso, sobre este domínio consideremos o Blowing-down das funções $\hat{w}_{-}, \tilde{w}, \hat{w}_{+} ;$dadas nas coordenadas $(x, \varepsilon, \mathcal{A})$ por

$$
\begin{aligned}
w_{-}(x, \varepsilon, \mathcal{A}) & :=|x|^{p} \hat{w}_{-}\left(|x|, \phi_{\hat{x}_{0}^{\prime}}^{-1}(\varepsilon, \mathcal{A})\right), \\
w_{\sim}(x, \varepsilon, \mathcal{A}) & :=\varepsilon^{\frac{p}{p+1}} \tilde{w}\left(x \varepsilon^{\frac{-1}{p+1}}, \varphi^{-1}(\varepsilon, \mathcal{A})\right), \\
w_{+}(x, \varepsilon, \mathcal{A}) & :=x^{p} \hat{w}_{+}\left(x, \phi_{\hat{x}_{1}^{\prime}}^{-1}(\varepsilon, \mathcal{A})\right) .
\end{aligned}
$$

Aqui usamos as relações $\varepsilon=\varepsilon^{p+1}, \quad \tilde{x}=x \tilde{\varepsilon}^{-1}$.

Desta construção, sobre o domínio $\tilde{\Gamma} \times \mathcal{O}_{\tilde{\Gamma}}$ definimos a função $C^{\infty}$

$$
\begin{aligned}
w: & \tilde{\Gamma} \times \mathcal{O}_{\tilde{\Gamma}} \longrightarrow \mathbf{R} \\
& (x, \Xi, \mathcal{A}) \longmapsto y=w(x, \Xi, \mathcal{A})
\end{aligned}
$$


como sendo

$$
w(x, \varepsilon, \mathcal{A}):= \begin{cases}w_{0}(x, \varepsilon, \mathcal{A}), & x \in \Gamma_{0}:=\left[x_{0}, x_{0}^{\prime}\right] \\ w_{-}(x, \varepsilon, \mathcal{A}), & x \in\left[x_{0}^{\prime}, 0\right) \\ w_{\sim}(x, \varepsilon, \mathcal{A}), & x=0 \\ w_{+}(x, \varepsilon, \mathcal{A}), & x \in\left(0, x_{1}^{\prime}\right] \\ w_{1}(x, \varepsilon, \mathcal{A}), & x \in \Gamma_{1}:=\left[x_{1}^{\prime}, x_{1}\right] .\end{cases}
$$

Claramente $w$ assim construída satisfaz os item (i), (ii) do enunciado; e o item (iii) decorre como conseqüência direta da forma da região Canard $\mathcal{O}_{\bar{\Gamma}}$. Isto mostra o Teorema.

No caso $(u, u)$, usamos o campo $-X$ sobre a condição inicial $i \in C_{\text {flat }}^{\infty}\left(\Gamma_{1} \times\right.$ $\left.\mathcal{O}_{1},\{\varepsilon=0\}, \widehat{W}_{x_{1}}\right)$. A construção do par $\left(\mathcal{O}_{\tilde{\Gamma}}, w\right)$ é completamente análoga ao caso $(s, s)$ (veja figuras 7.3 e 7.4). 


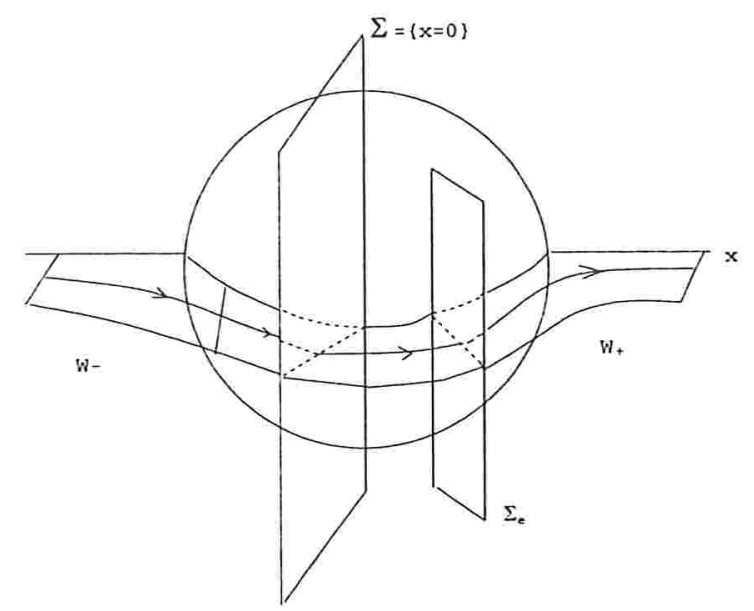

Figura 7.3: Superfície Canard no caso $(\mathrm{s}, \mathrm{s})$.

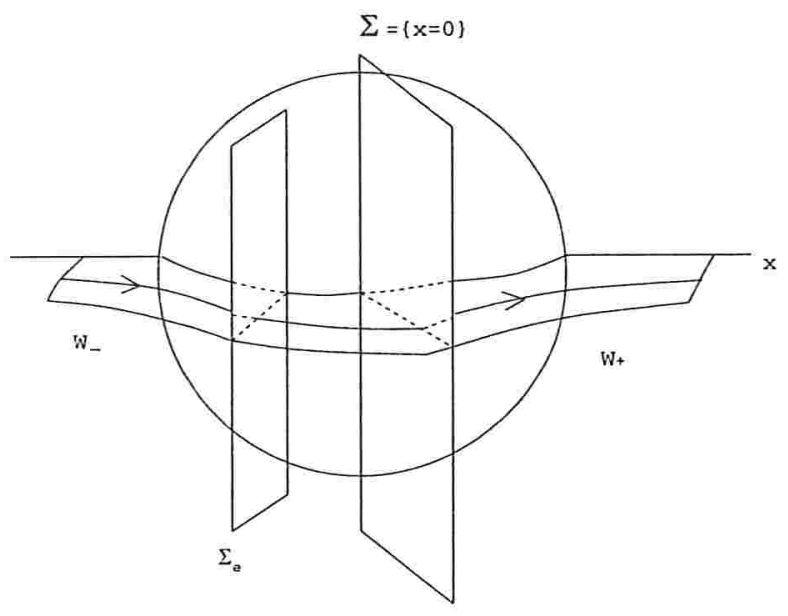

Figura 7.4: Superfície Canard no caso $(\mathrm{u}, \mathrm{u})$. 


\subsection{2 $\left(\mathcal{O}_{\widetilde{\Gamma}}, w\right)$ sobre o caso $(u, s)$}

Como no teorema anterior, temos o seguinte resultado:

Teorema 7.2.2 Suponha que o campo $X$ está no caso $(u, s)$. Então existem uma coleção de constantes estritamente positivas $\left\{m, r, k_{i}, l_{j}\right\}$ definindo a Região Canard $\mathcal{O}_{\widetilde{\Gamma}}$ como um conjunto aberto da forma

$$
\mathcal{O}_{\widetilde{\Gamma}}= \begin{cases}0<\varepsilon<r & \\ \left|a_{i}\right|<k_{i}|\varepsilon|^{\frac{2 p-i}{p+1}} & 0 \leq i \leq 2 p-1 \\ \left|b_{j}\right|<l_{j}|\varepsilon|^{\frac{p-j}{p+1}} & 0 \leq j \leq p-1 \\ \left|\mathcal{A}_{r}\right|<m & \end{cases}
$$

e uma função $C^{\infty}$

$$
\begin{array}{ll}
w: & \widetilde{\Gamma} \times \mathcal{O}_{\widetilde{\Gamma}} \longrightarrow \mathbf{R} \\
& (x,(\varepsilon, \mathcal{A})) \longmapsto y=w(x, \varepsilon, \mathcal{A})
\end{array}
$$

satisfazendo o seguinte:

(i) $w(0, \varepsilon, \mathcal{A}) \equiv 0$;

(ii) $W(\widetilde{\Gamma}):=\operatorname{graf}\{y=w(x, \varepsilon, \mathcal{A})\}$ é uma variedade invariante por $X$;

(iii) - w tem extensão continua ao fecho de $\widetilde{\Gamma} \times \mathcal{O}_{\tilde{\Gamma}}$, tal que $w(x, 0,0) \equiv$ 0 ;

- $w$ tem uma extensão blowing-up $C^{\infty}$ em $\{x=\varepsilon=0\}$.

\section{Demonstração}

Da observação anterior, este Teorema segue dos esquemas

$$
W\left(\Gamma_{-}\right) \longleftarrow^{\mathcal{B}_{-}>0} W_{-} \leftrightarrows W_{+} \longrightarrow^{\mathcal{B}_{+}<0} W\left(\Gamma_{+}\right)
$$

onde $\leftrightarrows$ significa que as variedades $W_{-}, W_{+}$se induzem uma da outra respectivamente (ver figura 7.5 ). Logo a existência de $\left(\mathcal{O}_{\widetilde{\Gamma}}, w\right)$ é detalhado como segue.

Passo 1 Do Lema 6.3.2, existe uma vizinhança aberta $U$ da origem $(\tilde{\varepsilon}, \tilde{\mathcal{A}})=$ $(0,0)$ sobre a carta $K_{\bar{\varepsilon}}$, e únicas variedlades centrais $W_{-}$em $P_{-}$e $W_{+}$ em $P_{+}$definidas sobre o domínio comum $N_{x}^{\prime} \times N_{e}^{\prime} \times V_{\mathcal{A}}^{\prime}$; onde

$$
N_{x}^{\prime}=\left[0, x_{0}^{\prime}\right], \quad N_{e}^{\prime}=\left(0, e_{0}^{\prime}\right), \quad V_{\mathcal{A}}^{\prime} \in\left(\mathbf{R}^{n}, 0\right) .
$$


Tal que sobre a secção transversal $\Sigma=\{x=0\}$ temos

$$
\begin{aligned}
W_{-} \cap \Sigma & =W_{+} \cap \Sigma=\operatorname{graf}\{y=Y(\tilde{\varepsilon}, \hat{\mathcal{A}})\} \\
U & \subset \mathcal{O}^{-} \cap \mathcal{O}^{+}=\mathcal{O}\left(W_{-}, W_{+}\right) .
\end{aligned}
$$

Onde $Y(\tilde{\varepsilon}, \hat{\mathcal{A}})$ é uma função $C^{\infty}$ arbitraria definida sobre $U \operatorname{com} Y(0,0)=$ 0 . Daqui segue o item $(i)$ para o caso particular $Y \equiv 0$ sobre alguma vizinhança da origem $(\tilde{\varepsilon}, \hat{\mathcal{A}})=(0,0)$.

Além disso, como no Teorema 7.2.1 podemos escolher $\left\{m, r, k_{i}, l_{j}\right\}$ tal que a inclusão $\Omega \subset \mathcal{O}\left(W_{-}, W_{+}\right)$seja estrita e definir a Região Canard como

$$
\mathcal{O}_{\widetilde{\Gamma}}:=\varphi(\Omega),
$$

o qual tem a forma do enunciado.

Passo 2 Da Proposição 7.1.4, podemos respectivamente estender as variedades centrais $W_{-}, W_{+}$únicamente como variedades centrais

$$
\begin{array}{lll}
W\left(\Gamma_{0}\right):=\operatorname{graf}\left\{y=w_{0}(x, \varepsilon, \mathcal{A})\right\}, & x \in \Gamma_{0}:=\left[x_{0}, x_{0}^{\prime}\right], \\
W\left(\Gamma_{1}\right):=\operatorname{graf}\left\{y=w_{1}(x, \varepsilon, \mathcal{A})\right\}, & x \in \Gamma_{1}:=\left[x_{1}^{\prime}, x_{1}\right] .
\end{array}
$$

Para funções $C^{\infty} w_{0}, w_{1}$ definidas sobre $\Gamma_{0} \times \mathcal{O}_{0}$ e $\Gamma_{1} \times \mathcal{O}_{1}$ respectivamente, onde

$$
\mathcal{O}_{0}:=N \cap \phi_{\hat{x}_{0}^{\prime}}\left(N_{e}^{\prime} \times V_{\hat{\mathcal{A}}}^{\prime}\right), \quad\left(\text { resp. } \quad \mathcal{O}_{1}:=N \cap \phi_{\hat{x}_{1}^{\prime}}\left(N_{e}^{\prime} \times V_{\hat{\mathcal{A}}}^{\prime}\right)\right)
$$

para alguma vizinhança $N$ da origem $(\varepsilon, \mathcal{A})=(0,0)$.

Controlando as constantes $\left\{m, r, k_{i}, l_{j}\right\}$, podemos obter a inclusão

$$
\mathcal{O}_{\widetilde{\Gamma}} \subset \mathcal{O}_{0} \cap \mathcal{O}_{1}
$$

Logo os item (ii), (iii) são satisfeitos, pela construção feita no Teorema 7.2 .1 da função $w \in C^{\infty}\left(\widetilde{\Gamma} \times \mathcal{O}_{\widetilde{\Gamma}}\right)$, oncle

$$
\widetilde{\Gamma}=\Gamma_{0} \cup\left[x_{0}^{\prime}, 0\right) \cup\{0\} \cup\left(0, x_{1}^{\prime}\right] \cup \Gamma_{1} \text {. }
$$




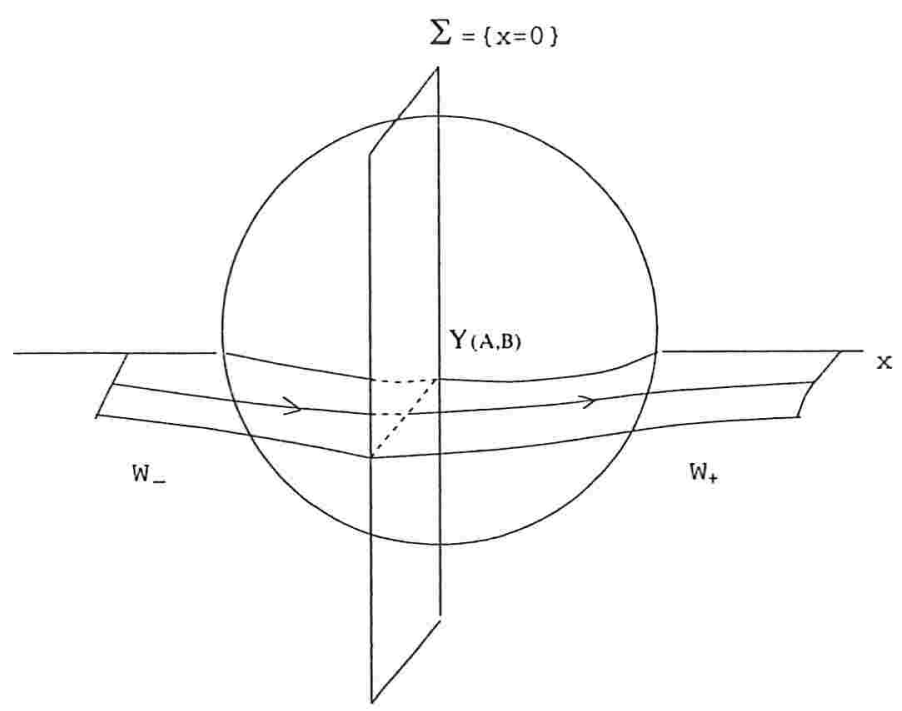

Figura 7.5: Superfície Canard no caso $(\mathrm{u}, \mathrm{s})$.

\subsection{3 $\left(\mathcal{O}_{\widetilde{\Gamma}}, w\right)$ sobre o caso $(s, u)$}

Finalmente vamos estudar o caso da transição $(s, u)$.

Teorema 7.2.3 Suponha que o campo $X$ está no caso $(s, u)$ e sejam

$$
\begin{aligned}
& i_{0} \in C_{\text {flat }}^{\infty}\left(V,\{\varepsilon=0\}, \widehat{W}_{x_{0}}\right), \\
& i_{1} \in C_{\text {flat }}^{\infty}\left(V,\{\varepsilon=0\}, \widehat{W}_{x_{1}}\right) .
\end{aligned}
$$

funções condição inicial arbitrárias para $X$ em $x=x_{0}$ e $x=x_{1}$ respectivamente. Então para cada indice par $s \in\{0,1,2, \ldots, 2 p-1\}$ existem:

1. Uma coleção de constantes estritamente positivas $\left\{m, r, k_{i}, l_{j}\right\}$ definindo um conjunto aberto da forma

$$
\mathcal{C}= \begin{cases}0<\varepsilon<r & \\ \left|a_{i}\right|<k_{i}|\varepsilon|^{\frac{2 p-i}{p+1}} & 0 \leq i \leq 2 p-1 \\ \left|b_{j}\right|<l_{j}|\equiv| \frac{p-j}{p+1} & 0 \leq j \leq p-1 \\ \left|\mathcal{A}_{r}\right|<m & ,\end{cases}
$$


2. Uma única subvariedade fechada de codimensão 1

$$
\mathcal{O}_{\tilde{\Gamma}}:=\operatorname{graf}\left\{a_{s}=\alpha_{s}\left(\varepsilon, \hat{a}, b, \mathcal{A}_{r}\right)\right\},
$$

onde $\alpha_{s}: \mathcal{C}_{s}:=\mathcal{C} \cap\left\{a_{s}=0\right\} \longrightarrow \mathbb{R}$ é uma função $C^{\infty}$ e $\hat{a}:=$ $\left(a_{0}, \ldots, a_{s-1}, a_{s+1}, \ldots, a_{2 p-1}\right)$.

3. Uma única função $C^{\infty}$

$$
\begin{aligned}
w: & \widetilde{\Gamma} \times \mathcal{O}_{\widetilde{\Gamma}} \longrightarrow \mathbb{R} \\
& (x,(\varepsilon, \mathcal{A})) \longmapsto y=w(x, \varepsilon, \mathcal{A})
\end{aligned}
$$

satisfazendo o seguinte

(i) $w\left(x_{k}, \varepsilon, \mathcal{A}\right)=i_{k}(\varepsilon, \mathcal{A}), \quad \forall(\varepsilon, \mathcal{A}) \in \mathcal{O}_{\widetilde{\Gamma}}, \quad k=0,1$;

(ii) $W(\widetilde{\Gamma}):=\operatorname{graf}\{y=w(x, \varepsilon, \mathcal{A})\}$ é uma variedade invariante por $X$;

(iii) $\alpha$ tem extensão blowing-up $C^{\infty}$ em $\{\varepsilon=0\}$;

(iv) - w tem extensão continua ao fecho de $\widetilde{\Gamma} \times \mathcal{O}_{\widetilde{\Gamma}}$, tal que $w(x, 0,0) \equiv$ 0 ;

- $w$ tem uma extensão blowing-up $C^{\infty}$ em $\{x=\varepsilon=0\}$.

\section{DEMONSTRAÇÃO}

Este resultado é mostrado pelo esquema seguinte

$$
W\left(\Gamma_{-}\right) \longrightarrow^{\mathcal{B}_{-}<0} W_{-} \text {ans } W_{+} \longleftarrow^{\mathcal{B}_{+}>0} W\left(\Gamma_{+}\right)
$$

onde $m \rightarrow$, significa que o contato das variedades centrais $W_{-}, W_{+}$é tal que sobre a seç̧ão transversal $\Sigma=\{x=0\}$ valem

- $W_{-} \cap \Sigma \equiv W_{+} \cap \Sigma$, ou

- $\Delta(\tilde{\varepsilon}, \tilde{\mathcal{A}}) \equiv 0, \quad \forall(\tilde{\varepsilon}, \tilde{\mathcal{A}}) \in \mathcal{O}_{\widetilde{\Gamma}}$.

Ver figuras 7.6 e 7.7 .

Mais precisamente os detalhes da construção de $\left(\mathcal{O}_{\tilde{\Gamma}}, w\right)$ e $\alpha_{s}$ são como segue: 
Passo 1 Da Proposição 7.1.3, segue que as variedades centrais $W\left(\Gamma_{0}\right), W\left(\Gamma_{1}\right)$ se estendem unicamente como variedades centrais

$$
\begin{array}{llll}
W_{-}:=\operatorname{graf}\left\{\hat{y}=\hat{w}_{-}(\hat{x}, e, \hat{\mathcal{A}})\right\}, & \text { em } & P_{-}, \\
W_{+}:=\operatorname{graf}\left\{\hat{y}=\hat{w}_{+}(\hat{x}, e, \hat{\mathcal{A}})\right\}, & \text { em } & P_{+},
\end{array}
$$

respectivamente definidas sobre domínios $N_{\hat{x}} \times N_{e} \times V_{\hat{\mathcal{A}}}$, onde, em cada caso,

$$
\begin{array}{ll}
N_{\hat{x}}:=\left[0, \hat{x}_{0}^{\prime}\right], & N_{e}=\left(0, e_{0}\right), \\
N_{\hat{x}}:=\left[0, \hat{x}_{1}^{\prime}\right], & \left.N_{e}=\left(0, e_{1}\right)\right), \\
V_{\hat{\mathcal{A}}} & \in\left(\mathbb{R}^{n}, 0\right) .
\end{array}
$$

Da definição das variedades $W\left(\Gamma_{0}\right), W\left(\Gamma_{1}\right)$, segue o item $(i)$. Os itens restantes serão satisfeitos após caracterização da Região de Colagem $\mathcal{O}\left(W_{-}, W_{+}\right)$.

Passo 2 Do Lema 6.3.4, existe um subconjunto aberto $U \subset \mathcal{O}^{-} \cap \mathcal{O}^{+}$vizinhança da origem $(\tilde{\varepsilon}, \tilde{\mathcal{A}})=(0,0)$, sobre a carta $K_{\bar{\varepsilon}}$.

Da Proposição 6.3.5, para cada índice par $s \in\{0,1,2, \ldots, 2 p-1\}$, existe uma pequena vizinhança $U_{s} \subset U$ da origem $(\tilde{\varepsilon}, \tilde{\mathcal{A}})=(0,0)$, e uma única função $C^{\infty}$

$$
a_{s}: U_{s} \cap\left\{A_{s}=0\right\} \longrightarrow \mathbb{R}, \quad a_{s}(0)=0,
$$

tal que a região de colagem $\mathcal{O}\left(W_{-}, W_{+}\right)$, é definida como

$$
\begin{aligned}
\mathcal{O}\left(W_{-}, W_{+}\right) \cap U_{s} & :=\operatorname{graf}\left\{A_{s}=a_{s}\left(\tilde{\varepsilon}, \hat{A}, B, \mathcal{A}_{r}\right)\right\}, \\
\hat{A} & :=\left(A_{0}, \ldots, A_{s-1}, A_{s+1}, \ldots, A_{2 p-1}\right) .
\end{aligned}
$$

Agora como no Teorema 7.2.1, escolhamos a coleção $\left\{m, r, k_{i}, l_{j}\right\}$, de maneira que a inclusão

$$
\Omega \subset U_{s}
$$

seja estrita, e além disso

$$
\mathcal{O}\left(W_{-}, W_{+}\right) \cap \Omega_{s}:=\operatorname{graf}\left\{A_{s}=a_{s} \mid \Omega_{s}\right\} \subset \Omega,
$$

onde

$$
\Omega_{s}:=\Omega \cap\left\{A_{s}=0\right\} \subset U_{s} \cap\left\{A_{s}=0\right\}
$$

Assim, basta definir

$$
\begin{aligned}
\mathcal{C} & :=\varphi(\Omega), \\
\mathcal{O}_{\widetilde{\Gamma}} & :=\varphi\left(\mathcal{O}\left(W_{-}, W_{+}\right) \cap \Omega_{s}\right) .
\end{aligned}
$$


Onde $\varphi$ é a função polinomial dada em (7.1).

E considerando o Blowing-down da função $a_{s}$, obtemos nas coordenadas $\left(\varepsilon, \hat{a}, b, \mathcal{A}_{r}\right)$ a função $\alpha_{s}$ definida por

$$
\alpha_{s}\left(\varepsilon, \hat{a}, b, \mathcal{A}_{r}\right):=\varepsilon^{\frac{2 p-s}{p+1}} a_{s} \circ \varphi^{-1}\left(\varepsilon, \hat{a}, b, \mathcal{A}_{r}\right) .
$$

Da construção feita no Teorema 7.2.1 para a função $w \in C^{\infty}\left(\widetilde{\Gamma} \times \mathcal{O}_{\widetilde{\Gamma}}\right)$, temos a existência de $\left(\mathcal{O}_{\widetilde{\Gamma}}, w\right)$ satisfazendo o enunciado deste Teorema. 


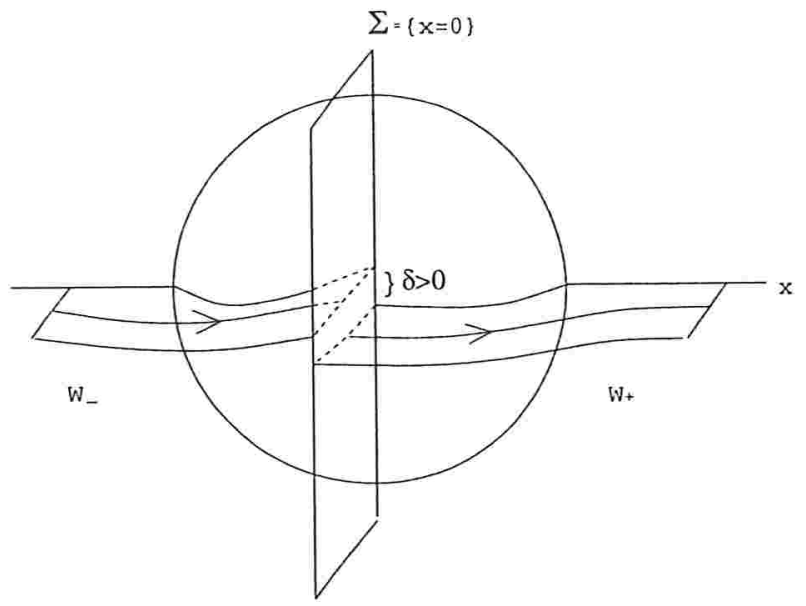

Figura 7.6: Transição Canard no caso $(\mathrm{s}, \mathrm{u})$.

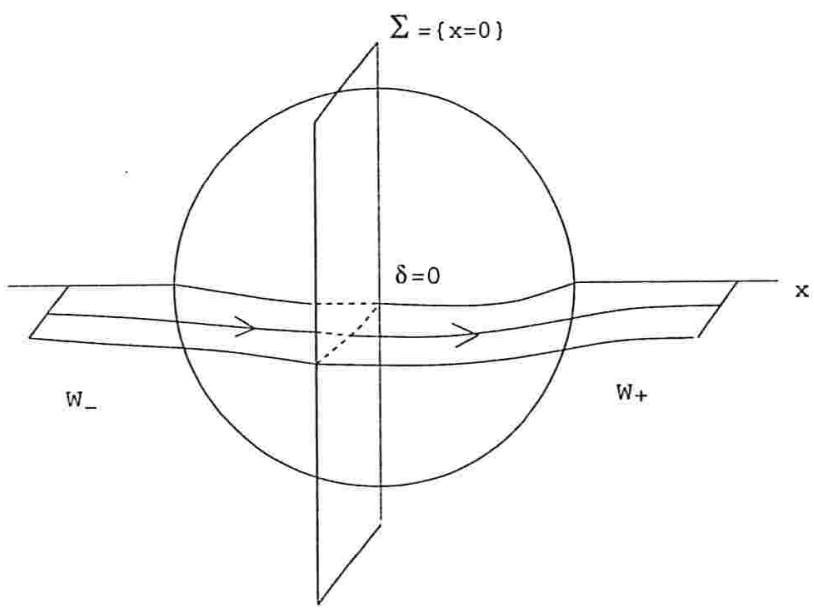

Figura 7.7: Superfície Canard no caso $(\mathrm{s}, \mathrm{u})$. 
Observação 7.2.4 No caso linear $\mathcal{Q}_{0}=0$, observemos que

- Nos casos $(s, s),(u, u),(u, s)$, a Região Canard $\mathcal{O}_{\tilde{\Gamma}}$ existe para uma arbitraria escolha das constantes $\left\{m, r, k_{i}, l_{j}\right\} ;$ com $m, r$ suficientemente pequenos.

- No caso $(s, u)$, a espressão da função distância $\delta(A, B)$ sobre o divisor excepcional $D_{0}=\left\{\varepsilon=\mathcal{A}_{r}=0\right\}$ é dada por

$\delta(A, B)=\left[C_{s}(B)+(-1)^{s} C_{s}(\bar{B})\right] A_{s}+\sum_{k=0, k \neq s}^{2 p-1}\left[C_{k}(B)+(-1)^{k} C_{k}(\bar{B})\right] A_{k}$.

De onde pelo teorema da função implícita fica definida a função

$$
A_{s}=a_{s}\left(\varepsilon, \hat{\mathcal{A}}, B, \mathcal{A}_{r}\right)
$$

dada no teorema anterior, que tem a seguinte expresão

$$
\left.a_{s}\right|_{D_{0}}=\frac{-1}{C_{s}(B)+(-1)^{s} C_{s}(\bar{B})} \sum_{k=0, k \neq s}^{2 p-1}\left[C_{k}(B)+(-1)^{k} C_{k}(\bar{B})\right] A_{k} .
$$

E em particular para s par e $B=\bar{B}=0$ temos

$$
\left.a_{s}\right|_{D_{0}}=\frac{-1}{c_{s}\left(\mathcal{B}_{0}\right)} \sum_{k=0, k \neq s}^{2 p-1} c_{k}\left(\mathcal{B}_{0}\right) A_{k}
$$

\subsection{Conclusões}

1. Se o conjunto de singularidades degeneradas $\operatorname{Deg}(X)$ para o campo $X$, satisfaz à Hipótese de Transversalidade (HT), então existem Superfícies Canard sobre $\operatorname{Deg}(X) \subset \Gamma$. Sendo $X$ o campo de vetores

$$
X(x, y)=y f(x, y) \frac{\partial}{\partial y} \in \mathcal{X}^{w}\left(\mathbb{R}^{2}, 0\right) .
$$

E $f(x, y)$ não divissivel por $y$.

2. A existência das Superfícies Canard para campos dados acima, é mostrada usando a Teoria Geométrica das Pert urbações Singulares (TGPS), a qual constitui a técnica principal neste trabalho para dar solução ao Problema Canard local (PCL). 
3. O uso da aplicação Blowing-up $\Phi$, constitui de fato uma ferramenta nova e de força relevante na pesquisa atual no meio matemático. Em particular o uso desta ferramenta em nosso trabalho (PCL) tive principal importância no estudo das Superfícies Canard para classes de campos de vetores dados acima. 


\section{Apêndice 1}

\subsection{Cálculo Assintótico}

Provaremos propriedades assintóticas sobre algumas funções conhecidas e estudaremos a convergência de algumas integrais impróprias.

\subsubsection{Escala de Comparação}

Uma vizinhança de $+\infty$ (resp. de $-\infty)$ é um intervalo da forma $[A,+\infty)$ (resp. $(-\infty, A])$, para alguma constante $A>0$. Temos por objetivo o estudo de funções continuas $f$ numa vizinhança de $+\infty$; para isto será preciso considerar o conjunto $\mathcal{E}$, que chamaremos Escala de Comparação o qual esta constituído por funções de um dos seguintes tipos:

$$
1, \quad x^{\alpha}(\alpha \neq 0), \quad(\log (x))^{\beta}(\beta \neq 0), \quad e^{c x^{\gamma}}(c \neq 0, \gamma>0)
$$

assim como também os produtos delas, tais como

$$
g(x)=x^{\alpha}(\log (x))^{\beta} e^{c x^{\gamma}}, \quad \alpha, \beta, c, \gamma \in \mathbb{R} .
$$

Este conjunto $\mathcal{E}$ satisfaz a seguintes propriedades:

(i) $g>0$, numa vizinhança de $+\infty$, para cada $g \in \mathcal{E}$,

(ii) $g=1$, ou $g \longrightarrow 0$, ou $g \longrightarrow \infty$, quando $x \longrightarrow \infty$, para cada $g \in \mathcal{E}$,

(iii) Se $h, g \in \mathcal{E}$, então $h g \in \mathcal{E}$ e $h / g \in \mathcal{E}$.

Com estas considerações poderíamos encontrar dois casos

1. Dada uma função contínua $f$, poderíamos encontrar funções $g \in \mathcal{E}$ conhecidas numa vizinhança de $+\infty$ tal que $f / g \longrightarrow c(c \neq 0$, finito), 
quando $x \longrightarrow \infty$.

Neste caso escreveremos

$$
f \sim c g, \quad \text { ou } \quad f(x) \sim c g(x)
$$

e chamaremos $c g$ a parte principal de $f$; no caso $c=1$, diremos que $f$ e $g$ são equivalentes, ficando claro a transitividade de $\sim$.

2. Também poderíamos encontrar funções $g \in \mathcal{E}$ conhecidas numa vizinhança de $+\infty$ tal que $f / g \longrightarrow 0$ ou $|f| / g \longrightarrow \infty$.

Exemplo 7.4.1 $x+\frac{1}{2} \sin (\pi x) \sim x$.

Para abreviar expressões numa vizinhança de $+\infty$, introduzimos notações devidas a Landau, relativas a $\mathcal{E}$.

Definição 7.4.2 Dada uma função contínua $f$ numa vizinhança de $+\infty$, $e$ $g \in \mathcal{E}$.

(i) Se $|f| / g$ é majorada por uma constante finita e diferente de zero, numa vizinhança de $+\infty$ escreveremos

$$
f=O(g)
$$

(ii) Se $f / g \longrightarrow 0$, quando $x \longrightarrow+\infty$, escreveremos

$$
f=o(g) .
$$

Observação 7.4.3 - Os casos particulares, $f=O(1)$ e $f=o(1)$ são oviamente entendidos,

- $f \sim c g \Longleftrightarrow f=c g+o(g)$ ou $f-c g=o(g)$.

Exemplo 7.4.4 $x \sin (x)=O(x), \quad x e^{-x}=o(x)$

Algumas propriedades relativas a $\mathcal{E}$ :

1. Se $f=O(g)$ e $g=O(h)$, então $f=O(h)$;

Se $f=o(g)$ e $g=O(h)$, então $f=o(h)$.

2. $O(g)+O(g)=O(g), \quad c . O(g)=O(g)$;

$o(g)+o(g)=o(g), \quad c . o(g)=o(g)$. 
3. $O\left(g_{1}\right) O\left(g_{2}\right)=O\left(g_{1} g_{2}\right), \quad o\left(g_{1}\right) O\left(g_{2}\right)=o\left(g_{1} g_{2}\right)$; $|O(g)|^{\lambda}=O\left(g^{\lambda}\right), \quad|o(g)|^{\lambda}=o\left(g^{\lambda}\right), \lambda>0$.

4. Se $f_{1} \sim c_{1} g$, e $f_{2} \sim c_{2} g,\left(c_{1}, c_{2} \neq 0\right), g \in \mathcal{E}$, então

$\left(f_{1}+f_{2}\right) \sim\left(c_{1}+c_{2}\right) g, \quad c_{1}+c_{2} \neq 0$

$f_{1}+f_{2}=o(g), \quad c_{1}+c_{2}=0$.

5. Se $f_{1} \sim c_{1} g_{1}$, e $f_{2} \sim c_{2} g_{2},\left(c_{1}, c_{2} \neq 0\right), g_{1}, g_{2} \in \mathcal{E}$, então

$\left(f_{1} f_{2}\right) \sim\left(c_{1} c_{2}\right)\left(g_{1} g_{2}\right), \quad f_{1} / f_{2} \sim\left(c_{1} / c_{2}\right)\left(g_{1} / g_{2}\right)$

$f^{\lambda} \sim c^{\lambda} g^{\lambda}, \quad(c>0, \lambda>0)$.

6. Se $f=o(g)$, e $g \longrightarrow+\infty$, quando $x \longrightarrow+\infty$, então para cada $\delta>0$

$$
\exp (-\delta g)=o(\exp (f)), \quad \exp (f)=o(\exp (\delta g))
$$

7. A relação ' $f=g$ ou $f=o(g)$ ' é de equivalência e constitui uma ordem total em $\mathcal{E}$.

\subsubsection{Convergência de Integrais Impróprias relativas a $\mathcal{E}$}

Para uma função contínua $f(>0)$ sobre $[a,+\infty)$, temos que para cada $x>a$, podemos escrever

$$
\int_{a}^{+\infty} f(t) d t=\lim _{x \rightarrow+\infty} F(x)
$$

onde

$$
F(x)=\int_{a}^{x} f(t) d t
$$

é tal que:

(i) F é monótona crescente;

(ii) $F^{\prime}(x)=f(x)$.

E portanto $\int_{a}^{+\infty} f(t) d t$ sempre existe. Tal integral é finita se e só se $F$ é limitada superiormente. 
Dos exemplos seguintes

$$
\begin{aligned}
\int_{a}^{x} t^{\alpha} d t & =\frac{1}{\alpha+1}\left(x^{\alpha+1}-a^{\alpha+1}\right), \alpha \neq-1 ; \\
\int_{a}^{x} t^{-1} d t & =\log (x)-\log (a) ; \\
\int_{a}^{x} t^{-1}(\log (t))^{\beta} d t & \left.=\frac{1}{\beta+1}(\log (x))^{\beta+1}-(\log (a))^{\beta+1}\right), \beta \neq-1 ; \\
\int_{a}^{x} t^{-1}(\log (t))^{-1} d t & =\log (\log x)-\log (\log a) ; \\
\int_{a}^{\infty} e^{c t^{\gamma}} d t & <\infty \text { se } c<0(>\infty \text { se } c>0) ;
\end{aligned}
$$

obtemos os seguintes critérios de convergência :

(i) Se $f(x)=O\left(x^{\alpha}(\log x)^{\beta}\right)$, com $\alpha \leq-1$, e $\beta<-1$, então a integral $\int_{a}^{\infty} f(t) d t$ é finita.

(ii) Se $f(x) \geq x^{\alpha}(\log x)^{\beta}$, com $\alpha \geq-1$, e $\beta \geq-1$, então a integral $\int_{a}^{\infty} f(t) d t$ é infinita.

(iii) Se $f(x)=O\left(e^{c t^{\gamma}}\right), c<0$, então $\int_{a}^{\infty} f(t) d t$ é finita;

Se $f(x) \geq e^{c t^{\gamma}}, c>0$, então $\int_{a}^{\infty} f(t) d t$ é infinita.

Exemplo 7.4.5 Para a função $f(t)=t^{x-1} e^{-t}$, a integral imprópria $\int_{1}^{+\infty} f(t) d t$, é finita para cada $x$ real, pois $f \in \mathcal{E}$ e $e^{-t} \longrightarrow 0, t \longrightarrow \infty$.

Analogamente para integrais de funções sobre $(a, b]$

$$
\int_{a}^{b} f(t) d t=\lim _{h \longrightarrow 0} \int_{a+h}^{b} f(t) d t
$$

Se o limite existir será chamada integral imprópria de $f$ em $a$.

Exemplo 7.4.6 Para a função $f(t)=t^{x-1} e^{-t}$, a integral imprópria em 0

$$
\int_{0}^{1} f(t) d t \sim \int_{0}^{1} t^{x-1} d t=\lim _{h \longrightarrow 0} \int_{h}^{1} t^{x-1} d t=\lim _{h \longrightarrow 0}\left(\frac{1}{x}-\frac{h^{x}}{x}\right)
$$

é finita se $x>0$. 
para $x>0$, a integral

$$
\Gamma(x)=\int_{0}^{+\infty} t^{x-1} e^{-t} d t
$$

é finita. E conseqüentemente também a integral

$$
\int_{0}^{+\infty} t^{\alpha} e^{-c t^{\beta}} d t, \quad c>0, \quad \beta \neq 0, \alpha \in \mathrm{N}
$$

a qual, é igual a:

$$
\left(\beta c^{\frac{\alpha+1}{\beta}}\right)^{-1} \Gamma\left(\frac{\alpha+1}{\beta}\right) .
$$

Proposição 7.4.7 Sejam $f, g$ duas funções contínuas por partes em $[a,+\infty)$ e $g>0$ sobre $[a,+\infty)$. Então

(a) Se $\int_{a}^{+\infty} g(t) d t=+\infty$, então numa vizinhança de $+\infty$ :
a.1) Se $f=O(g)$, então $\int_{a}^{x} f(t) d t=O\left(\int_{a}^{x} g(t) d t\right)$;
a.2) Se $f=o(g)$, então $\int_{a}^{x} f(t) d t=o\left(\int_{a}^{x} g(t) d t\right)$;
a.3) Se $f \sim c g\left(c \neq 0\right.$, constante), então $\left.\int_{a}^{x} f(t) d t \sim c \int_{a}^{x} g(t) d t\right)$.

(b) Se $\int_{a}^{+\infty} g(t) d t$ é finita, então numa vizinhança de $+\infty$ :

b.1) Se $f=O(g)$, então $\int_{x}^{+\infty} f(t) d t=O\left(\int_{x}^{+\infty} g(t) d t\right)$;

b.2) Se $f=o(g)$, então $\int_{x}^{+\infty} f(t) d t=o\left(\int_{x}^{+\infty} g(t) d t\right)$;

b.3) Se $f \sim c g\left(c \neq 0\right.$, constante), então $\left.\int_{x}^{+\infty} f(t) d t \sim c \int_{x}^{+\infty} g(t) d t\right)$.

\section{DEMONSTRAÇÃo}

b.2) $f=o(g)$ então, para cada $\varepsilon>0$, existe $x_{0}=x_{0}(\varepsilon)>a$ tal que para $x \geq x_{0},|f(x)| \leq \varepsilon g(x)$. Logo do teorema do valor médio.

$$
\left|\int_{x_{0}}^{x} f(t) d t\right| \leq \varepsilon \int_{x_{0}}^{x} g(t) d t
$$


Como $\int_{a}^{x} g(t) d t$ é finita, então $\int_{x_{0}}^{x} g(t) d t$ é também finita quando $x \longrightarrow$ $+\infty$. Assim para $x \geq x_{0}$ :

$$
\begin{aligned}
\left|\int_{x}^{+\infty} f(t) d t\right| & =\left|\int_{x_{0}}^{+\infty} f(t) d t-\int_{x_{0}}^{x} f(t) d t\right| \\
& \leq \lim _{b \rightarrow+\infty}\left|\int_{x_{0}}^{b} f(t) d t\right|+\left|\int_{x_{0}}^{x} f(t) d t\right| \\
& \leq \varepsilon\left(\lim _{b \rightarrow+\infty} \mid \int_{x_{0}}^{b} g(t) d t+\int_{x}^{x_{0}}-g(t) d t\right) \\
& \leq \varepsilon\left(\int_{x_{0}}^{+\infty} g(t) d t+\int_{x}^{x_{0}} g(t) d t\right) \\
& =\varepsilon \int_{x}^{+\infty} g(t) d t .
\end{aligned}
$$

b.1) É análogo

b.3) Como $f \sim c g$ é equivalente a $f-c g=o(g)$, temos por (b.2) que (b.3) é verdadeiro.

Observação 7.4.8 Seja $g>0$, continuamente diferenciável, $\frac{1}{x}=o\left(\left|\frac{g^{\prime}(x)}{g(x)}\right|\right)$, e suponha que $g^{\prime}(x)$ não muda de sinal numa vizinhança de $+\infty$.

(i) $\operatorname{Se} g^{\prime}(x)>0$ então $g / g^{\prime}$ está definida numa vizinhança de $+\infty$. Da proposição anterior, a hipótese implica que

$$
\log (x)=o(\log (|g(x)|))
$$

Como $g>0$ e $g^{\prime}>0, g(x) \longrightarrow+\infty$, logo $\log (|g(x)|) \longrightarrow+\infty$, numa vizinhança de $+\infty$; assim pela observação 6 da seção anterior, existe $\alpha>0$ tal que

$$
e^{-\alpha \log (|g(x)|)}=o\left(e^{\log (x)}\right), \quad e^{\log (x)}=o\left(e^{\alpha \log (|g(x)|)}\right) .
$$

Assim vale a relação

$$
x^{1 / \alpha}=o(g(x)), \quad 1 / \alpha>0,
$$

e a integral $\int_{a}^{+\infty} g(t) d t$ é infinita. 
(ii) Se $g^{\prime}(x)<0$, a condição $\frac{1}{x}=o\left(-\frac{g^{\prime}(x)}{g(x)}\right)$, implica imediatamente (pela proposição anterior) que

$$
\log (x)=o\left(\log \left(\frac{1}{g(x)}\right)\right)
$$

como $g^{\prime}<0$ e $g>0, \quad \log \left(\frac{1}{g(x)}\right) \longrightarrow+\infty, \quad x \longrightarrow+\infty$. Analogamente existe $\beta>0$, tal que numa vizinhança de $+\infty$, temos

$$
e^{-\beta \log (1 / g(x))}=o\left(e^{\log (x)}\right), \quad e^{\log (x)}=o\left(e^{\beta \log (1 / g(x))}\right),
$$

e vale a relação

de onde a integral $\int_{a}^{+\infty} g(t) d t$ é finita.

$$
g(x)=o\left(x^{-1 / \beta}\right), \quad 1 / \beta>0,
$$

(iii) $S e h^{\prime}(x)=o(1)$, a proposição anterior implica que $h(x)=o(x)$.

Proposição 7.4.9 Nas condições da observação anterior para $g(x)$ e $g^{\prime}(x)$; suponha que $h(x)=\frac{g(x)}{g^{\prime}(x)}$ é continuamente diferenciável na vizinhança de $+\infty$ e satisfaz $h^{\prime}(x)=o(1)$. Então

(i) $\operatorname{Se} g^{\prime}(x)>0$ numa vizinhança de $+\infty$, a integral $\int_{a}^{+\infty} g(t) d t$, é infinita $e$

$$
\int_{a}^{x} g(t) d t \sim \frac{(g(x))^{2}}{g^{\prime}(x)}
$$

(ii) Se $g^{\prime}(x)<0$ numa vizinhança de $+\infty$, a integral $\int_{a}^{+\infty} g(t) d t$, é finita $e$

$$
\int_{x}^{+\infty} g(t) d t \sim \frac{(g(x))^{2}}{\left|g^{\prime}(x)\right|} .
$$

\section{DEMONSTRAÇÃO}

(ii) Como $g^{\prime}(x)<0$ e $h^{\prime}(x)=o(1)$, da observação anterior temos que a integral $\int_{a}^{+\infty} g(t) d t$ é finita e

$$
\int_{x}^{+\infty} g(t) d t \sim \int_{a}^{+\infty}\left(1+h^{\prime}(t)\right) g(t) .
$$

Integrando por partes, esta última integral é igual a

$$
\begin{aligned}
& =\lim _{b \rightarrow+\infty}\left(b \cdot g(b) \cdot \frac{h(b)}{b}\right)-g(x) h(x) \\
& =\frac{(g(x))^{2}}{-g^{\prime}(x)} \\
& =\frac{(g(x))^{2}}{\left|g^{\prime}(x)\right|}
\end{aligned}
$$


(i) É similar.

Exemplo 7.4.10 1 1) Na integral $\int_{x}^{+\infty} e^{-t^{2}}$, as funções $g(t)=e^{-t^{2}}, g^{\prime}(t)=$ $-2 t e^{-t^{2}}, h(t)=-1 / 2 t$, e $h^{\prime}(t)=1 / 2 t^{2}$, satisfazem as hipóteses da proposição; logo vale a relação

$$
\int_{x}^{+\infty} e^{-t^{2}} \sim \frac{e^{-x^{2}}}{2 x}
$$

2) Na integral $\int_{x}^{+\infty} t^{q} e^{-c t^{p}} d t,(c>0)$, as funções $g(x)=x^{q} e^{-c x^{p}}, g^{\prime}(x)=$ $g(x)\left(q x^{-1}-c p x^{p-1}\right), h(x)=\frac{1}{q x^{-1}-c p x^{p-1}} e h^{\prime}(x)=\frac{q+c p(p-1) x^{p}}{\left(q-c p x^{p}\right)^{2}}$; satisfazem as hipóteses da proposição; assim como $\frac{1}{x}=o(1)$, vale a relação

$$
\begin{aligned}
\int_{x}^{+\infty} t^{q} e^{-c t^{p}} d t & \sim \frac{g(x)^{2}}{g^{\prime}(x)} \\
& =\frac{g(x)^{2}}{\left|g(x)\left(q x^{-1}-c p x^{p-1}\right)\right|} \\
& =\frac{g(x)}{\left|q x^{-1}-c p x^{p-1}\right|} \\
& \sim \frac{g(x)}{\left|-c p x^{p-1}\right|} \\
& =\frac{x^{q} e^{-c x^{p}}}{\left|\left(-c x^{p}\right)^{\prime}\right|} .
\end{aligned}
$$

Observação 7.4.11

- Uma opção alternativa no último exemplo seria usar integração por partes várias vezes no segundo termo da igualdade:

$$
\int_{x}^{+\infty} t^{q} e^{-c t^{p}} d t=\frac{x^{q} e^{-c x^{p}}}{c p x^{p-1}}-\int_{x}^{+\infty} e^{-c t^{p}} d\left(\frac{t^{q}}{-c p t^{p-1}}\right)
$$

diminuindo as potências $x^{q}$, e comprovando que

$$
\int_{x}^{+\infty} e^{-c t^{p}} d\left(\frac{t^{q}}{-c p t^{p-1}}\right)=o\left(\frac{x^{q} e^{-c x^{p}}}{c p x^{p-1}}\right)
$$

- Para $g(x)=x^{q} e^{P(x)}$, onde $P(x)=c_{1} x^{p_{1}}+c_{2} x^{p_{2}}+\ldots+c_{k} x^{p_{k}}$, com $c_{1}<0$, $p_{1}>p_{2}>\ldots>p_{k}$, vale a relação

$$
\int_{x}^{+\infty} t^{q} e^{P(t)} d t \sim \frac{x^{q} e^{P(x)}}{\left|P^{\prime}(x)\right|}
$$




\subsection{Equações Diferenciais}

Veremos alguns conceitos elementares da teoria das equações diferencias ordinárias com a finalidade de simplificar o desenvolvimento do trabalho. Seja $I=I\left(t_{0}, y_{0}\right) \subseteq \mathbb{R}$ um intervalo e $\Omega \subseteq \mathbb{R} \times \mathbb{R}^{n}$ um conjunto aberto; consideremos a matriz real $A(t) \in M(n \times n)$ contínua e definida em $t \in I$. Em geral estaremos interessados em estudar equações diferenciais da forma

$$
y^{\prime}=A(t) y+N(t, y)
$$

onde $N(t, y)$ é uma função contínua sobre $\Omega$.

É conhecido que se $N(t, y)=D(t)$ é um vetor de $M(n \times 1)$, para cada $\left(t_{0}, y_{0}\right) \in I \times \mathbf{R}^{\mathrm{n}}$ existe uma única solução $\varphi(t)=\varphi\left(t, t_{0}, y_{0}\right)$, definida em $I$, do problema de Cauchy $y^{\prime}=A(t) y+D(t), \quad y\left(t_{0}\right)=y_{0}$.

Vejamos outras propriedades destas soluções

Lema 7.5.1 Sejam, $\Phi(t), \Psi(t)$ soluções fundamentais da equação diferencial $y^{\prime}=A(t) y$. Então existe uma matriz constante não singular $C$, tal que $\Psi(t)=\Phi(t) C$.

\section{DEMONSTRAÇÃO}

Esta propriedade decorre trivialmente da igualdade $\left(\Psi(t)^{-1} \Phi(t)\right)^{\prime}=0$.

Neste caso uma solução fundamental é escrita como $\exp \left(\int_{t_{0}}^{t} A(\tau) d \tau\right)$.

Lema 7.5.2 Sejam, $\Phi(t)$ uma solução fundamental da equação diferencial $y^{\prime}=A(t) y$. Então a solução $\varphi(t)=\varphi\left(t, t_{0}, x_{0}\right)$, do problema de Cauchy

$$
y^{\prime}=A(t) y+D(t), \quad y\left(t_{0}\right)=y_{0}
$$

é dada pela fórmula

$$
\varphi\left(t, t_{0}, x_{0}\right)=\Phi(t)\left\{\Phi^{-1}\left(t_{0}\right) x_{0}+\int_{t_{0}}^{t} \Phi^{-1}(\tau) D(\tau) d \tau\right\} .
$$

\section{DEMONSTRAÇÃO}

Pelo lema anterior, podemos colocar $\varphi(t)=\Phi(t) C(t)$; a substituição desta igualdade na equação não homogênea implica resolver o problema de Cauchy

$$
C^{\prime}(t)=\Phi^{-1}(t) D(t), \quad C\left(t_{0}\right)=\Phi^{-1}\left(t_{0}\right) x_{0}
$$

De onde decorre a demonstração. 
Exemplo 7.5.3 Para funções contínuas $p(x), q(x)$ de valor real, definidas sobre $I\left(x_{0}, y_{0}\right) \subseteq \mathbb{R}$; considere o problema de Cauchy

$$
\mathbf{B}(m)=\left\{\begin{array}{l}
y^{\prime}=p(x) y+q(x) y^{m}, \quad m \in \mathbf{N} \\
y\left(x_{0}\right)=y_{0} .
\end{array}\right.
$$

Tomando $z=y^{1-m}$, este problema é equivalente a

$$
\left\{\begin{array}{l}
z^{\prime}=(1-m) p(x) z+(1-m) q(x), \quad m \in \mathrm{N} \\
z\left(x_{0}\right)=z_{0}=y_{0}^{1-m}
\end{array}\right.
$$

Como $\Phi(x)=\exp \left((1-m) \int_{x_{0}}^{x} p(t) d t\right)$, é a solução fundamental de

$$
z^{\prime}=(1-m) p(x) z
$$

Então usando os lemas anteriores temos que a solução de $\mathbf{B}(m)$ é dada pela fórmula

$$
y\left(x, x_{0}, y_{0}\right)=\left[\frac{\Phi^{-1}(x)}{\frac{1}{y_{0}^{m-1}}+(1-m) \int_{x_{0}}^{x} \Phi^{-1}(t) q(t) d t}\right]^{\frac{1}{m-1}} .
$$

Em particular se por exemplo $p(x)=\sum_{l=0}^{N} c_{l} x^{l}$ e $q(x)=\sum_{k=0}^{M} d_{k} x^{k}$, são dois polinômios reais, então teríamos

$$
y\left(x, x_{0}, y_{0}\right)=\left[\frac{\exp ((m-1) P(x))}{\frac{1}{y_{0}^{m-1}}+(1-m) \sum_{k=0}^{M} d_{k} J_{k}(x)}\right]^{\frac{1}{m-1}}
$$

onde

$$
P(x)=\sum_{l=0}^{N} c_{k} \frac{x^{l+1}}{l+1}-\sum_{l=0}^{N} c_{k} \frac{x_{0}^{l+1}}{l+1}, \quad J_{k}(x)=\int_{x_{0}}^{x} t^{k} \exp ((m-1) P(t)) d t
$$




\section{Bibliografia}

[AK] Al Kelley; The Stable, Center-Stable, Center, Center-Unstable, Unstable Manifolds; Journal of Differential Equations 3, 546-570(1967).

[CLW] Shui Nee Chow, Chengzhi Li, Duo Wang; Normal Forms and Bifurcation of Planar Vector Fields; Cambridge University Press, 1994.

[DP] Daniel Panazzolo; On the Existence of Canard Solutions; Publicacions Matemàtiques, Vol.44(2000), 503-592.

[FD] Freddy Dumortier; Singularities of Vector Fields on the Plane; Journal of Differential Equations 23, 53-106(1977).

[FDR1] F.Dumortier, R.Roussarie; Geometric singular perturbation theory beyond normal hyperbolicity; Prepublication ou Rapport de Recherche, no 163(1998), France.

[FDR2] Freddy Dumortier, Robert Roussarie; Canard Cycles and Center Manifold; Mem. Amer. Math. Soc., 121(577)(1996).

[HW] H.Whitney; Analytic Extensions of Differentiable Functions Defined in Closet Sets; Trans. Amer. Soc., 36(1)(1934), 63-89.

[JD] Jean Dieudonné; Infinitesimal Calculus; Hermann Paris, 1971.

[JS] Jan Sijbrand; Properties of Center Manifolds; Trans. Amer. Math. Soc., 289(2)(1985), 431-469.

[KS] M Krupa and P Szmolyan; Extending Slow Manifolds near Transcritical and Pitchfork Singularities; Nonlinearity 14(2001) 1473-1491.

[M] Bernard Malgrange; Ideals of Differentiable Functions; Tata Institute of Fundamental Research, Oxford University Press, Bombay(India), 1966. 
[NF] Neil Fenichel; Geometric Singular Perturbation theory for Ordinary Differential Equation; Journal of Differential Equations, 31, 5398(1979).

[S] Sotomayor J.; Lições de Equações Diferenciais Ordinarias; Projeto Euclides IMPA, 1979.

[T] Floris Takens; Singularities of Vector Fields; Institut des Hautes Études Scientifiques; Publications Mathématiques, No 43(47-100), 1974.

[WE] Wiktor Eckhaus; Relaxation Oscillations Including a Standar Chase on French Ducks; In Asymptotic Analysis II, Springer Lect. Notes in Math. 985, 449-494, 1983. 